\title{
Fan-extensions in fragile matroids
}

\author{
Carolyn Chun* \\ Department of Mathematics \\ Brunel University \\ United Kingdom \\ carolyn.chun@brunel.ac.uk \\ Deborah Chun ${ }^{\dagger}$ \\ College of Engineering \& Sciences \\ Institute of Technology, West Virginia University \\ U.S.A. \\ deborah.chun@mail.wvu.edu

\section{Dillon Mayhew ${ }^{\ddagger}$} \\ School of Mathematics, Statistics and Operations Research \\ Victoria University of Wellington \\ New Zealand \\ dillon.mayhew@msor . vuw.ac.nz \\ Stefan H. M. van Zwam ${ }^{\S}$ \\ Department of Mathematics \\ Louisiana State University \\ U.S.A. \\ svanzwam@math. lsu.edu
}

Submitted: Dec 29, 2013; Accepted: May 10, 2015; Published: May 22, 2015

Mathematics Subject Classifications: 05B35

\begin{abstract}
If $\mathcal{S}$ is a set of matroids, then the matroid $M$ is $\mathcal{S}$-fragile if, for every element $e \in E(M)$, either $M \backslash e$ or $M / e$ has no minor isomorphic to a member of $\mathcal{S}$. Excluded-minor characterizations often depend, implicitly or explicitly, on understanding classes of fragile matroids. In certain cases, when $\mathcal{M}$ is a minor-closed class of $\mathcal{S}$-fragile matroids, and $N \in \mathcal{M}$, the only members of $\mathcal{M}$ that contain $N$ as a minor are obtained from $N$ by increasing the length of fans. We prove that if this is the case, then we can certify it with a finite case-analysis. The analysis involves examining matroids that are at most two elements larger than $N$.
\end{abstract}

Keywords: matroids; excluded minors; fragility; fans

\footnotetext{
*Supported by NSF International Research Fellowship Program grant \#OISE0967050.

†Supported by an NSF Vertical Integration of Research \& Education travel grant.

${ }^{\ddagger}$ Supported by a Rutherford Discovery Fellowship.

${ }^{\S}$ Supported by NSF grant \#1501985.
} 


\section{Introduction}

Let $\mathcal{S}$ be a set of matroids. When we say that a matroid has an $\mathcal{S}$-minor, we mean that it has a minor isomorphic to a member of $\mathcal{S}$. The matroid $M$ is $\mathcal{S}$-fragile if, for every element $e \in E(M)$, either $M \backslash e$ or $M / e$ has no $\mathcal{S}$-minor. Note that every minor of an $\mathcal{S}$-fragile matroid is also $\mathcal{S}$-fragile. Fragility has been studied at various times under different names: Oxley examined non-binary $\left\{U_{2,4}\right\}$-fragile matroids [11]; Truemper proved a constructive characterization of a class of binary $\left\{F_{7}, F_{7}^{*}\right\}$-fragile matroids [13]; and Kingan and Lemos have made a study of binary $\left\{F_{7}, F_{7}^{*}, M^{*}\left(K_{3,3}\right), M^{*}\left(K_{5}\right)\right\}$-fragile matroids $[6,7]$.

Our study of fragile matroids is motivated by the goal of finding new excluded-minor characterizations. The matroid $S$ is a strong stabilizer for the partial field $\mathbb{P}$, if, roughly speaking, every $\mathbb{P}$-representation of $S$ extends uniquely to a $\mathbb{P}$-representation of any $\mathbb{P}$-representable matroid that contains $S$ as a minor. More information on strong stabilizers can be found in [3] or [12]. Understanding $\mathcal{S}$-fragile matroids, where $\mathcal{S}$ is a set of strong stabilizers, has been important in excluded-minor characterizations. For example, $U_{2,4}$ is a strong stabilizer for both $\mathrm{GF}(4)$ and the near-regular partial field. The excluded-minor characterizations of GF(4)-representable [4] and near-regular matroids [5] both implicitly use the fact that a non-binary 3-connected GF(4)-representable matroid is $\left\{U_{2,4}\right\}$-fragile if and only if it is a whirl. Geelen, Gerards, and Whittle conjecture that, for any prime power $q$, and any $\operatorname{GF}(q)$-representable matroid $N$, there is an integer $k$ such that every $\mathrm{GF}(q)$-representable $\{N\}$-fragile matroid has branch width at most $k$ [2, Conjecture 5.9]. In another application of the link between fragility and excluded-minor results, Mayhew, Van Zwam, and Whittle [8] have shown that this conjecture implies that there are only finitely many excluded minors for $\mathrm{GF}(5)$-representability.

In our study of $\mathcal{S}$-fragile matroids, $\mathcal{S}$ will typically be a set of excluded minors for representability over some partial field. This allows us to assume certain properties of $\mathcal{S}$. In particular, we can assume that the members of $\mathcal{S}$ are 3 -connected and contain at least four elements. We say that the matroid $N$ is 3 -connected up to series and parallel sets if it is connected, and $\min \{r(X), r(Y)\}=1$ or $\min \left\{r^{*}(X), r^{*}(Y)\right\}=1$ for every 2-separation $(X, Y)$ of $N$. The next result follows immediately from [6, Proposition 3.1].

Proposition 1.1. Let $\mathcal{S}$ be a collection of 3-connected matroids, each of which has at least four elements. Assume that $N$ is $\mathcal{S}$-fragile and that $N$ has an $\mathcal{S}$-minor. Then $N$ is 3-connected up to series and parallel sets.

It is a fairly easy exercise to show that wheels and whirls are representable over every partial field, except that whirls are not representable over $\mathrm{GF}(2)$ and the regular partial field. Therefore we will henceforth assume that $\mathcal{S}$ contains no wheels or whirls. This implies that any matroid with an $\mathcal{S}$-minor is neither a wheel nor a whirl.

A fan is a sequence of elements, where consecutive sets of three elements alternately form triads and triangles. In some cases, the only way to construct $\mathbb{P}$-representable $\mathcal{S}$-fragile matroids by building from a matroid $N$ is to increase the length of fans in $N$. For example, Figure 1(i) shows the rank-6 binary matroid $N_{12}$. This matroid is obtained by gluing three copies of $M\left(K_{4}\right)$ to $F_{7}$ along three lines that contain a common point 
$p$, and then deleting the points of intersection, apart from three that lie in a common line avoiding $p$. The matroid in Figure 1(ii) is obtained from $N_{12}$ by lengthening the fan $\left(u_{1}, u_{2}, u_{3}, u_{4}\right)$ to $\left(u_{1}, a, b, u_{2}, u_{3}, u_{4}\right)$, and by lengthening $\left(v_{1}, v_{2}, v_{3}, v_{4}\right)$ to $\left(v_{1}, v_{2}, v_{3}, v_{4}, c\right)$. Any matroid obtained by lengthening fans in this way is a fan-extension of $N_{12}$. (We delay the formal definition of fan-extensions until Section 2.) Any 3-connected binary matroid that is $\left\{F_{7}, F_{7}^{*}\right\}$-fragile and contains $N_{12}$ as a minor is obtained from $N_{12}$ by lengthening the three disjoint 4-element fans. The resulting family of matroids is essentially the same as the family $\mathcal{F}_{1}(m, n, r)$, as described in [6]. This result, and other applications of our main theorem, will be described in Section 4. These applications bring the excluded-minor characterisations for matroids representable over the partial fields $\mathbb{H}_{5}$ and $\mathbb{U}_{2}$ within reach.

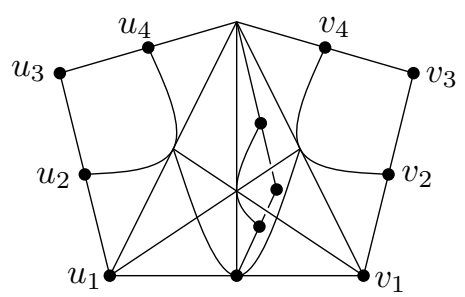

(i)

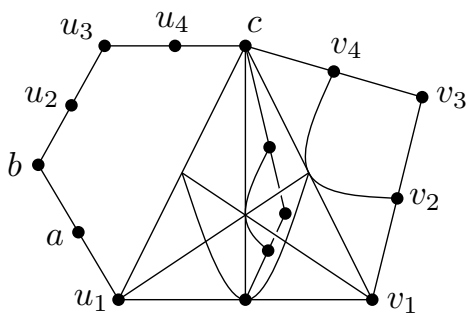

(ii)

Figure 1: $N_{12}$, and one of its fan-extensions.

Suppose we are given a minor-closed class, $\mathcal{M}$, and a matroid $N \in \mathcal{M}$. We would like to know whether $M \in \mathcal{M}$ being 3-connected with $N$ as a minor implies that $M$ is a fanextension of $N$. Our main theorem allows us to use a finite case-analysis to check whether this implication is true. From now on we make no mention of fragility. Theorem 1.2 instead uses the conditions implied by Proposition 1.1, and the assumption that $\mathcal{S}$ does not contain any wheels or whirls.

Theorem 1.2. Let $\mathcal{M}$ be a set of matroids closed under isomorphism and minors. Let $N \in \mathcal{M}$ be a 3-connected matroid such that $|E(N)| \geqslant 4$ and $N$ is neither a wheel nor a whirl. Assume that any member of $\mathcal{M}$ with $N$ as a minor is 3-connected up to series and parallel sets. If there is a 3-connected matroid in $\mathcal{M}$ with $N$ as a minor that is not a fan-extension of $N$, then there exists such a matroid, $M$, satisfying $|E(M)|-|E(N)| \leqslant 2$.

This is restated as Theorem 6.10 later in the paper. The assumptions on $\mathcal{M}$ and $N$ are justified if $\mathcal{M}$ is a minor-closed class of $\mathcal{S}$-fragile matroids, and $N$ is a member of $\mathcal{M}$ with an $\mathcal{S}$-minor, where we make the assumption that the members of $\mathcal{S}$ are 3 -connected with at least four elements, and $\mathcal{S}$ contains no wheel or whirl.

To see that the bound $|E(M)|-|E(N)| \leqslant 2$ is best possible, we let $G$ be one of the graphs drawn schematically in Figure 2. If $G$ is the graph on the left, let $N=M(G) \backslash x \backslash y$. If $G$ is the graph on the right, let $N=M(G) / x \backslash y$. In either case, let $M=M(G)$. Now set $\mathcal{M}$ to be the class containing all minors of $M$ and their isomorphs. We note that $M$ 
is not a fan-extension of $N$ relative to the fans $\left(u_{1}, u_{2}, u_{3}\right)$ and $\left(v_{1}, v_{2}, v_{3}\right)$. However, any member of $\mathcal{M}$ that has $N$ as a minor and that is at most one element larger than $N$ is a fan-extension.
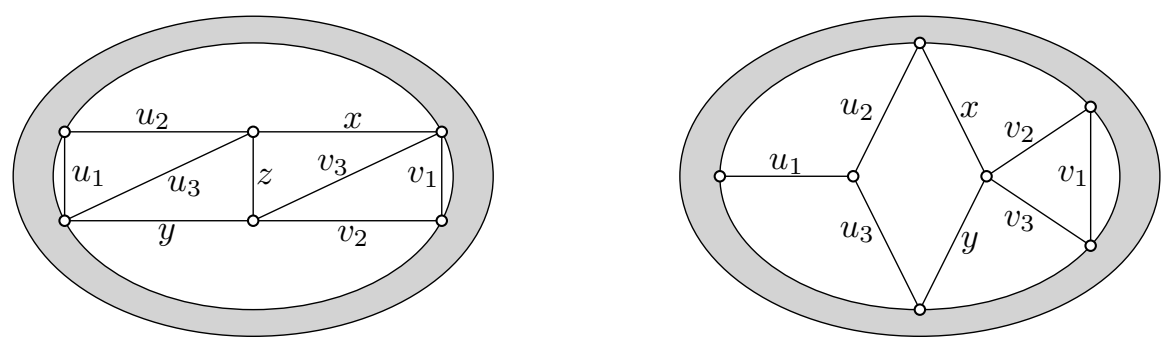

Figure 2: Schematic drawings of two graphs.

In Section 2 we state some definitions, including that of a fan-extension. Section 3 is dedicated to an alternative formulation of fan-extensions, based upon the idea of gluing wheels to a 'core' matroid. We use this alternative formulation in Section 4, where we sketch some applications of our theorem to binary $\left\{F_{7}, F_{7}^{*}\right\}$-fragile matroids and $\left\{U_{2,5}, U_{3,5}\right\}$-fragile matroids that are representable over the partial field $\mathbb{H}_{5}$. The proof of the main theorem is contained in Sections 5 and 6.

\section{Fan-extensions}

Any unexplained terminology or notation that we use can be found in Oxley [9].

Definition 2.1. Let $M$ be a matroid. A fan of $M$ is an ordered sequence, $\left(e_{1}, \ldots, e_{n}\right)$, of $n \geqslant 3$ distinct elements such that

$$
\left\{e_{1}, e_{2}, e_{3}\right\},\left\{e_{2}, e_{3}, e_{4}\right\}, \ldots,\left\{e_{n-2}, e_{n-1}, e_{n}\right\}
$$

is an alternating sequence of triangles and triads.

If $\left\{e_{1}, e_{2}, e_{3}\right\}$ is a triangle, then the elements with odd indices in $\left(e_{1}, \ldots, e_{n}\right)$ are spoke elements, and elements with even indices are rim elements. These labels are reversed if $\left\{e_{1}, e_{2}, e_{3}\right\}$ is a triad. We sometimes blur the distinction between ordered and unordered sets where doing so creates no problems. So for example, we may talk about fans being disjoint. Note that $\left(e_{1}, \ldots, e_{n}\right)$ is a fan of $M$ if and only if it is a fan of $M^{*}$. If $1<$ $i<n$, then $e_{i}$ is an internal element of the fan, otherwise it is a terminal element. In a 3-connected matroid with at least five elements, no triangle can be a triad, so the partitioning into spoke and rim elements is unambiguous. In particular, in a 3-connected matroid with at least four elements that is not a whirl, an element in a fan is either a spoke element or a rim element, and not both. We frequently replace the ordering $\left(e_{1}, \ldots, e_{n}\right)$ with $\left(e_{n}, \ldots, e_{1}\right)$. We call this process reversing. A contiguous subsequence of $\left(e_{1}, \ldots, e_{n}\right)$ is a subsequence of the form $\left(e_{s}, e_{s+1}, \ldots, e_{t-1}, e_{t}\right)$, where $1 \leqslant s \leqslant t \leqslant n$. If $F=\left(e_{1}, \ldots, e_{n}\right)$ and $F^{\prime}=\left(e_{1}^{\prime}, \ldots, e_{m}^{\prime}\right)$ are two sequences, then we say that $F$ is consistent 
with $F^{\prime}$ if $\left(e_{1}, \ldots, e_{n}\right)$ is a subsequence (not necessarily contiguous) of either $\left(e_{1}^{\prime}, \ldots, e_{m}^{\prime}\right)$ or $\left(e_{m}^{\prime}, \ldots, e_{1}^{\prime}\right)$. If $F=\left(e_{1}, \ldots, e_{n}\right)$ is an ordered sequence of elements, and $X \subseteq\left\{e_{1}, \ldots, e_{n}\right\}$, then $F-X$ is the subsequence produced from $F$ by omitting all elements in $X$. As usual, we abbreviate the singleton set $\{x\}$ to $x$. We say that the fan $F=\left(e_{1}, \ldots, e_{n}\right)$ is maximal if there is no fan $\left(e_{1}^{\prime}, \ldots, e_{m}^{\prime}\right)$ such that $\left\{e_{1}^{\prime}, \ldots, e_{m}^{\prime}\right\}$ properly contains $\left\{e_{1}, \ldots, e_{n}\right\}$.

Now we formally define fan-extensions. To avoid disrupting the exposition, we will relegate some technical lemmas until later sections. Let $M$ be a 3 -connected matroid with a fan $\left(e_{1}, \ldots, e_{n}\right)$, where $n \geqslant 4$. If $e_{1}$ is a spoke element, and $M \backslash e_{1}$ is 3 -connected, then clearly $\left(e_{2}, \ldots, e_{n}\right)$ is a fan of $M \backslash e_{1}$, and $M$ is said to be obtained from $M \backslash e_{1}$ by a fanlengthening move on this fan. Similarly, if $e_{1}$ is a rim element, and $M / e_{1}$ is 3-connected, then $M$ is obtained from $M / e_{1}$ by a fan-lengthening move on $\left(e_{2}, \ldots, e_{n}\right)$. If $n \geqslant 5$, and $e_{i}$ is a rim element, where $1 \leqslant i \leqslant n-1$, and $M / e_{i} \backslash e_{i+1}$ is 3 -connected, then Proposition 5.2 shows $\left(e_{1}, \ldots, e_{i-1}, e_{i+2}, \ldots, e_{n}\right)$ is a fan of $M / e_{i} \backslash e_{i+1}$, and $M$ is said to be obtained from $M / e_{i} \backslash e_{i+1}$ by a fan-lengthening move on this fan. Note that $M$ is obtained by a fanlengthening move on $M^{\prime}$ if and only if $M^{*}$ is obtained by a fan-lengthening move on $\left(M^{\prime}\right)^{*}$ (applied to the reversed fan, in the case that $|E(M)|=\left|E\left(M^{\prime}\right)\right|+2$ ). Moreover, $M$ is necessarily 3 -connected.

Let $N$ be a 3 -connected matroid with at least four elements. Let $\mathcal{F}_{N}$ be a collection of pairwise disjoint fans in $N$. Note that we do not require fans in $\mathcal{F}_{N}$ to be maximal. A fan in $N$ could potentially contain many fans in $\mathcal{F}_{N}$ as subsequences. If $M^{\prime}$ has $N$ as a minor, and $\mathcal{F}^{\prime}$ is a family of fans of $M^{\prime}$, then we say that $\mathcal{F}^{\prime}$ is a covering family of $M^{\prime}$ (relative to $N$ and $\mathcal{F}_{N}$ ) if the following conditions are satisfied:

(i) the fans in $\mathcal{F}^{\prime}$ are pairwise disjoint,

(ii) $\left|\mathcal{F}^{\prime}\right|=\left|\mathcal{F}_{N}\right|$,

(iii) for every $F_{N} \in \mathcal{F}_{N}$, there is a fan $F^{\prime} \in \mathcal{F}^{\prime}$ such that $F_{N}$ is consistent with $F^{\prime}$,

(iv) every element in $E\left(M^{\prime}\right)-E(N)$ is contained in one of the fans in $\mathcal{F}^{\prime}$.

Observe that the fan $F^{\prime}$ in condition (iii) may contain elements of $E(N)$ that are not in $F_{N}$. If we reverse any fan in a covering family, we obtain another covering family. Informally, a fan-extension of $N$ is obtained by finding a covering family, applying a fanlengthening move to one of the fans in that family, and then reiterating this process. More formally, we have:

Definition 2.2. We define $N$ to be a fan-extension of $N$. We recursively define the set of fan-extensions of $N$ (relative to $\mathcal{F}_{N}$ ) to be the smallest set satisfying the following condition:

- if $M^{\prime}$ is a fan-extension of $N$, and $\mathcal{F}^{\prime}$ is a covering family of $M^{\prime}$ containing the fan $F^{\prime}$, then any matroid obtained from $M^{\prime}$ by a fan-lengthening move on $F^{\prime}$ is a fan-extension of $N$ (relative to $\mathcal{F}_{N}$ ).

If $M$ is obtained from $M^{\prime}$ by lengthening $F^{\prime}$ into the fan $F$, then $\left(\mathcal{F}^{\prime}-\left\{F^{\prime}\right\}\right) \cup\{F\}$ is a covering family of $M$. Therefore the next result follows easily from the definition. 
Proposition 2.3. Let $N$ be a 3-connected matroid with at least four elements and let $\mathcal{F}_{N}$ be a collection of pairwise disjoint fans in $N$. If $M$ is a fan-extension of $N$, then $M$ is 3-connected, has $N$ as a minor, and has a covering family.

Note that $M$ is a fan-extension of $N$ relative to $\mathcal{F}_{N}$ if and only if $M^{*}$ is a fan-extension of $N^{*}$ relative to $\mathcal{F}_{N}$. The converse of Proposition 2.3 need not hold. In Lemma 6.11, we essentially construct a 3-connected matroid that is not a fan-extension, although it does have $N$ as a minor and a covering family. However, we will have occasion to use the partial converse in Corollary 2.5. If $\mathcal{F}$ is a covering family of $M$, then we say that $\mathcal{F}$ admits a fan-shortening move if $M$ is obtained from $M^{\prime}$ by using a fan-lengthening move on $F^{\prime} \in \mathcal{F}^{\prime}$ to produce the fan $F$, where $F \in \mathcal{F}$ and $M^{\prime}$ has $N$ as a minor.

Proposition 2.4. Assume $N$ is neither a wheel nor a whirl, and that no fan in $N$ contains two distinct fans in $\mathcal{F}_{N}$ (considered as unordered sets). Let $M$ be a 3-connected matroid with $N$ as a minor, and assume that every minor of $M$ that has $N$ as a minor is 3-connected up to series and parallel sets. Let $\mathcal{F}$ be a covering family of $M$. If $M \neq N$, then $\mathcal{F}$ admits a fan-shortening move.

Proof. Let $\left(e_{1}, \ldots, e_{n}\right)$ be an arbitrary fan in $\mathcal{F}$, and assume that the internal element $e_{i}$ belongs to $E(M)-E(N)$. By duality, we can assume that $e_{i}$ is a rim element. If $N$ is a minor of $M \backslash e_{i}$, then $n \leqslant 4$, for otherwise $M \backslash e_{i}$ contains a triangle that contains a series pair, and this contradicts the hypotheses of the proposition (see Proposition 6.2). But if $n \leqslant 4$, the three elements in $\left\{e_{1}, e_{2}, e_{3}, e_{4}\right\}-e_{i}$ all belong to a fan in $\mathcal{F}_{N}$, and as this set contains a series pair in $M \backslash e_{i}$, it follows that $N$ is not cosimple, a contradiction. Therefore $N$ is a minor of $M / e_{i}$. As $\left\{e_{i-1}, e_{i+1}\right\}$ is a parallel pair in $M / e_{i}$, we can reverse $\left(e_{1}, \ldots, e_{n}\right)$ as necessary, and assume that $N$ is a minor of $M / e_{i} \backslash e_{i+1}$. Thus $n \geqslant 5$, as $\left(e_{1}, \ldots, e_{n}\right)$ must contain three elements from $E(N)$. Proposition 6.4 implies that $M / e_{i} \backslash e_{i+1}$ is 3-connected, and we can set $M^{\prime}$ to be $M / e_{i} \backslash e_{i+1}$. Now Proposition 5.2 implies $\left(e_{1}, \ldots, e_{i-1}, e_{i+2}, \ldots, e_{n}\right)$ is a fan of $M^{\prime}$. As $M$ is obtained from $M^{\prime}$ by performing a fan-lengthening move on this fan, we are done. Therefore we will now assume that the internal elements of fans in $\mathcal{F}$ all belong to $E(N)$.

As $M \neq N$, we can reverse as necessary, and let $\left(e_{1}, \ldots, e_{n}\right)$ be a fan in $\mathcal{F}$ where $e_{1}$ belongs to $E(M)-E(N)$. By duality, we can assume that $e_{1}$ is a spoke element. If $M \backslash e_{1}$ is 3-connected, then we set $M^{\prime}$ to be $M \backslash e_{1}$ and we are done. Therefore we assume that $M \backslash e_{1}$ is not 3 -connected, and thus contains a series pair. Now $e_{1}$ is contained in a triad, $T^{*}$, of $M$. Orthogonality with the triangle $\left\{e_{1}, e_{2}, e_{3}\right\}$ means that $T^{*}$ contains $e_{2}$ or $e_{3}$. Let $x$ be the element in $T^{*}-\left\{e_{1}, e_{2}, e_{3}\right\}$. Because $\left(e_{1}, \ldots, e_{n}\right)$ contains at least three elements of $E(N), n \geqslant 4$, so $e_{2}$ and $e_{3}$ are internal elements, and hence belong to $E(N)$. As either $\left\{x, e_{2}\right\}$ or $\left\{x, e_{3}\right\}$ is a series pair in $M \backslash e_{1}$, we see $N$ is a minor of $M \backslash e_{1} / x$. Because $\mathcal{F}$ is a covering family, $x$ is contained in a fan in $\mathcal{F}$. Because $x$ is not in $E(N)$, it is not an internal element, so $x$ is a terminal element of a fan in $\mathcal{F}$. Orthogonality with $T^{*}$ shows that it is a rim element. It cannot be the case that $x$ is in $\left(e_{1}, \ldots, e_{n}\right)$, for then $x=e_{n}$, so this fan would contain a triad that does not consist of three consecutive elements. The dual of Proposition 5.6 shows that this is a contradiction. Assume that 
$x=f_{m}$, where $\left(f_{1}, \ldots, f_{m}\right)$ is a fan in $\mathcal{F}$. If $M / f_{m}$ is 3 -connected we are done, so we assume $f_{m}$ is in a triangle, $T$. This triangle must contain either $f_{m-2}$ or $f_{m-1}$, and an element from $T^{*}$. Orthogonality with the triad $\left\{e_{2}, e_{3}, e_{4}\right\}$ shows that $T$ cannot contain $e_{2}$ or $e_{3}$, so it contains $e_{1}$.

If $T=\left\{f_{m-1}, f_{m}, e_{1}\right\}$ and $T^{*}=\left\{f_{m}, e_{1}, e_{2}\right\}$, then $\left(f_{1}, \ldots, f_{m}, e_{1}, \ldots, e_{n}\right)$ is a fan of $M$ that contains two fans in $\mathcal{F}_{N}$. Let $\left(x_{1}, \ldots, x_{p}\right)$ be the subsequence obtained from $\left(f_{1}, \ldots, f_{m}, e_{1}, \ldots, e_{n}\right)$ by omitting the elements not in $E(N)$. (Any such elements have to be in $\left\{f_{1}, f_{m}, e_{1}, e_{n}\right\}$.) It follows from [9, Corollary 8.2.5] that any three consecutive elements in $\left(x_{1}, \ldots, x_{p}\right)$ are 3 -separating in $N$, and therefore form either a triangle or a triad in $N$. It is not too difficult to see, using orthogonality, that either $\left(x_{1}, \ldots, x_{p}\right)$ is a fan in $N$, or $\min \left\{r_{N}\left(\left\{x_{1}, \ldots, x_{p}\right\}\right), r_{N}^{*}\left(\left\{x_{1}, \ldots, x_{p}\right\}\right)\right\} \leqslant 2$. In the former case, $N$ has a fan that contains two distinct fans from $\mathcal{F}_{N}$, so we have a contradiction to the hypotheses. Therefore $\left\{x_{1}, \ldots, x_{p}\right\}$ is a line in either $N$ or $N^{*}$. However, $\left\{e_{2}, e_{3}, e_{4}\right\}$ is a triad of $M$, and of $N$ also (since $\left\{e_{2}, \ldots, e_{n-1}\right\} \subseteq E(N)$ and $\left\{e_{2}, \ldots, e_{n}\right\}$ contains at least three elements of $E(N))$. Similarly, $\left\{f_{m-3}, f_{m-2}, f_{m-1}\right\}$ is a triangle of $N$, so $\left\{f_{m-3}, f_{m-2}, f_{m-1}, e_{2}, e_{3}, e_{4}\right\}$ cannot be contained in a line of $N$ or $N^{*}$.

This disposes of the case that $T=\left\{f_{m-1}, f_{m}, e_{1}\right\}$ and $T^{*}=\left\{f_{m}, e_{1}, e_{2}\right\}$. Next we assume that $T \neq\left\{f_{m-1}, f_{m}, e_{1}\right\}$. A symmetrical argument will then deal with the case that $T^{*} \neq\left\{f_{m}, e_{1}, e_{2}\right\}$. Because $T$ is not $\left\{f_{m-1}, f_{m}, e_{1}\right\}$, it is $\left\{f_{m-2}, f_{m}, e_{1}\right\}$ instead. This means that $m=4$, for otherwise we violate orthogonality between $T$ and $\left\{f_{m-4}, f_{m-3}, f_{m-2}\right\}$. If $T^{*}=\left\{f_{m}, e_{1}, e_{2}\right\}$, then $\left(f_{1}, f_{3}, f_{2}, f_{4}, e_{1}, e_{2}, \ldots, e_{n}\right)$ is a fan of $M$ that contains two fans of $\mathcal{F}_{N}$. If $T^{*}$ is not $\left\{f_{m}, e_{1}, e_{2}\right\}$, then it is $\left\{f_{m}, e_{1}, e_{3}\right\}$, which implies that $n=4$. In this case $\left(f_{1}, f_{3}, f_{2}, f_{4}, e_{1}, e_{3}, e_{2}, e_{4}\right)$ is a fan of $M$ that contains two fans in $\mathcal{F}_{N}$. In either case, we can obtain a contradiction to the hypotheses of the proposition, exactly as in the previous paragraph. This completes the proof.

Corollary 2.5. Let $M$ and $N$ be as described in Proposition 2.4. If $M$ contains a covering family, then it is a fan-extension of $N$ relative to $\mathcal{F}_{N}$.

Proof. The proof is by induction on $|E(M)|-|E(N)|$. If $M=N$, then $M$ is a fanextension of $N$, as desired. Therefore we assume $M \neq N$. Let $\mathcal{F}$ be a covering family of $M$. By Proposition 2.4, $M$ is obtained from some matroid $M^{\prime}$ by a performing a fanlengthening move on $F^{\prime}$ to obtain $F \in \mathcal{F}$. As $(\mathcal{F}-\{F\}) \cup\left\{F^{\prime}\right\}$ is a covering family of $M^{\prime}$, it follows by induction that $M^{\prime}$ is a fan-extension of $N$. Now the result is immediate.

\section{A wheel-gluing lemma}

In this section we develop an alternative description of fan-extensions that will be of use in Section 4, where we describe some applications of our main theorem. Intuitively, a family of fans in $N$ can be seen as the result of gluing wheels along a family of triangles in a matroid that is smaller than $N$. A fan-extension of $N$ can be obtained in the same way, except that we glue on larger wheels. The object of this section is to make these ideas formal. Our focus here will be on the case that $N$ is representable, although it would be possible to extend these results to arbitrary matroids. 
Proposition 3.1. Let $M_{1}$ and $M_{2}$ be matroids on the same ground set. Assume $\left(e_{1}, e_{2}, e_{3}, e_{4}\right)$ is a fan with $e_{1}$ as a spoke element in both $M_{1}$ and $M_{2}$. If $M_{1} \backslash e_{1}=M_{2} \backslash e_{1}$, then $M_{1}=M_{2}$.

Proof. Assume that $M_{1} \neq M_{2}$. Without loss of generality, we can assume that $X$ is a circuit in $M_{1}$, but an independent set in $M_{2}$. Then $e_{1}$ must be contained in $X$. As $M_{1} \backslash e_{1} / e_{2}=M_{2} \backslash e_{1} / e_{2}$, and $M_{i} / e_{2}$ is obtained from $M_{i} \backslash e_{1} / e_{2}$ by adding the element $e_{1}$ parallel to $e_{3}$ (for $i=1,2$ ), it follows that $M_{1} / e_{2}=M_{2} / e_{2}$. Therefore $e_{2}$ is not in $X$. Now $M_{1} / e_{2} \backslash e_{3}=M_{2} / e_{2} \backslash e_{3}$, and $M_{i} \backslash e_{3}$ is obtained from $M_{i} / e_{2} \backslash e_{3}$ by adding $e_{2}$ in series to $e_{4}$. Therefore $M_{1} \backslash e_{3}=M_{2} \backslash e_{3}$, so $e_{3} \in X$. By strong circuit-exchange between $X$ and $\left\{e_{1}, e_{2}, e_{3}\right\}$, there is a circuit, $C$, in $M_{1}$ contained in $\left(X-e_{1}\right) \cup e_{2}$ that contains $e_{2}$. As $C$ does not contain $e_{1}$, it is also a circuit of $M_{2}$. Now $C$ and $\left\{e_{1}, e_{2}, e_{3}\right\}$ are distinct circuits of $M_{2}$, both of which are contained in $X \cup e_{2}$ and both of which contain $e_{2}$. As $X$ is independent in $M_{2}$, this is a contradiction.

Let $M_{1}$ and $M_{2}$ be matroids with $E\left(M_{1}\right) \cap E\left(M_{2}\right)=T$. The generalised parallel connection of $M_{1}$ and $M_{2}$ is defined if $M_{1}\left|T=M_{2}\right| T$, and $T$ is a modular flat of $M_{2}$. In this case, we use the notation $M_{1} \otimes_{T} M_{2}$ to denote the generalized parallel connection. Note that a triangle in a simple binary matroid is a modular flat. The flats of $M_{1} \otimes_{T} M_{2}$ are precisely the subsets $F \subseteq E\left(M_{1}\right) \cup E\left(M_{2}\right)$ such that $F \cap E\left(M_{i}\right)$ is a flat of $M_{i}$, for $i=1,2$ (see [9, Proposition 11.4.13]). Assume $M_{1}, M_{2}$, and $M_{3}$ are matroids with $E\left(M_{2}\right) \cap$ $E\left(M_{3}\right) \subseteq E\left(M_{1}\right)$. Let $T_{i}=E\left(M_{1}\right) \cap E\left(M_{i}\right)$ for $i=2,3$. If $M_{1} \otimes_{T_{2}} M_{2}$ and $M_{1} \otimes_{T_{3}} M_{3}$ are both defined, then it follows easily from the definition that $\left(M_{1} \otimes_{T_{2}} M_{2}\right) \otimes_{T_{3}} M_{3}$ and $\left(M_{1} \otimes_{T_{3}} M_{3}\right) \bigotimes_{T_{2}} M_{2}$ are defined and equal.

Let $N_{0}$ be a matroid and let $\mathcal{T}=\left\{T_{i}\right\}_{i \in I}$ be a multiset of triangles of $N_{0}$, indexed by the set $I=\{1, \ldots, t\}$. Note that triangles in $\mathcal{T}$ need not be disjoint, nor indeed distinct. These are the triangles to which we will glue wheels. For each $i \in I$, let $T_{i}=\left\{a_{i}, b_{i}, c_{i}\right\}$. The end points of the fan that we generate by the gluing operation will be $a_{i}$ and $c_{i}$. Let $r$ be a function from $I$ to $\{2,3,4,5, \ldots\}$. This function determines the rank of the wheels that we glue to the triangles in $\mathcal{T}$. Finally, let $X$ be a subset of $\cup_{k \in I} T_{k}$ such that, for all $i \in I, b_{i} \notin X$ implies $b_{i}=a_{j}$ or $b_{i}=c_{j}$ for some $j \in I$. After gluing the wheels to the triangles in $\mathcal{T}$, we delete the set $X$. We call the tuple $\left(N_{0}, \mathcal{T}, r, X\right)$ a blueprint.

Assume that $\left(N_{0}, \mathcal{T}, r, X\right)$ is a blueprint. For each $i \in I$, we let the matroid $W_{i}$ be a copy of a wheel with rank $r(i)$. The ground set of $W_{i}$ will be $\left\{x_{1}^{i}, \ldots, x_{r(i)}^{i}, y_{1}^{i}, \ldots, y_{r(i)}^{i}\right\}$, where we make the identifications $x_{1}^{i}=a_{i}, y_{r(i)}^{i}=b_{i}$, and $x_{r(i)}^{i}=c_{i}$. The ground set of $W_{i}$ is labeled in such a way that $\left(x_{1}^{i}, y_{1}^{i}, x_{2}^{i}, y_{2}^{i}, \ldots, x_{r(i)}^{i}, y_{r(i)}^{i}\right)$ is a fan with $x_{1}^{i}$ as a spoke element. Moreover $\left\{x_{1}^{i}, y_{r(i)}^{i}, x_{r(i)}^{i}\right\}$ is a triangle. Thus $E\left(W_{i}\right) \cap E\left(W_{j}\right) \subseteq \cup_{k \in I} T_{k}$ when $i \neq j$. For each $i \in I=\{1, \ldots, t\}$, we recursively define $N_{i}$ to be $N_{i-1} \bigotimes_{T_{i}} W_{i}$. By an earlier observation, the ordering of the indices in $I$ makes no difference to the definition of $N_{t}$. We define $\otimes\left(N_{0}, \mathcal{T}, r, X\right)$ to be $N_{t} \backslash X$, and we say that this matroid is obtained by gluing wheels to $N_{0}$. From the definition of generalized parallel connection, it is straightforward to show that $\left(x_{1}^{i}, y_{1}^{i}, x_{2}^{i}, \ldots, y_{r(i)-1}^{i}, x_{r(i)}^{i}\right)-X$ is a fan in $\otimes\left(N_{0}, \mathcal{T}, r, X\right)$, for every $i \in I$. We call such a fan a canonical fan of $\otimes\left(N_{0}, \mathcal{T}, r, X\right)$. 
Henceforth we take $N$ to be a 3-connected representable matroid that is neither a wheel nor a whirl. Let $E$ be the ground of $N$. We assume that $|E| \geqslant 4$, so that $N$ is simple. If $X$ and $Y$ are disjoint subsets of $E(N)$, then $\sqcap(X, Y)=r(X)+r(Y)-r(X \cup Y)$. Given an embedding of $N$ in a projective geometry, the parameter $\sqcap(X, Y)$ tells us the rank of the maximal subspace spanned by both $X$ and $Y$. The next two results are standard, and not difficult to prove. We omit their proofs.

Proposition 3.2. Let $F=\left(e_{1}, \ldots, e_{n}\right)$ be a fan of $N$. If $e_{1}$ is a rim element, then $\sqcap\left(\left\{e_{1}, e_{2}\right\}, E-F\right)=1$.

Proposition 3.3. Let $F=\left(e_{1}, \ldots, e_{n}\right)$ be a fan of $N$, and let $R$ be the set of rim elements in $F$. Then $\sqcap(R, E-F)=1$.

Recall that we have required $N$ to be representable, so henceforth we identify elements of $E$ with points in a projective geometry, $P$. Let $\mathcal{F}=\left\{F_{i}\right\}_{i \in I}$ be a family of pairwise disjoint fans in $N$ indexed by the set $I=\{1, \ldots, t\}$. As $N$ is neither a wheel nor a whirl, Proposition 5.4 implies that the complement of any fan $F_{i}$ contains at least two elements. We are going to apply Propositions 3.2 and 3.3 to each fan in $\mathcal{F}$ and its reversal. This identifies three points in $P$ that are distinguished by the fan. We add three elements in parallel to these points. To be more precise, we let $F_{i}=\left(e_{1}, \ldots, e_{n}\right)$ be a fan in $\mathcal{F}$. If $e_{1}$ is a spoke element, then we add $a_{i}$ in parallel to $e_{1}$. If $e_{1}$ is a rim element, then we add $a_{i}$ in parallel to the single point in $P$ that is in the closure of $E-F_{i}$ and $\left\{e_{1}, e_{2}\right\}$. Note that if $e_{1}$ is a spoke element, and $e_{1}$ is not in the closure of $E-F_{i}$, then it is in the closure and the coclosure of $\left\{e_{2}, \ldots, e_{n}\right\}$. This implies $\lambda\left(F_{i}\right) \leqslant 1$, which contradicts the 3 -connectivity of $N$. Therefore $a_{i}$ is in the closure of $E-F_{i}$ in any case. Similarly, we add $c_{i}$ in parallel to $e_{n}$ if $e_{n}$ is a spoke element, and otherwise we add $c_{i}$ in parallel to the point of $P$ that lies in the closure of both $E-F_{i}$ and $\left\{e_{n-1}, e_{n}\right\}$. Let $R$ be the set of rim elements in $F_{i}$. Add $b_{i}$ in parallel to the point of $P$ that is in the closure of both $R$ and $E-F_{i}$. Proposition 5.1 implies that $\lambda\left(F_{i}\right)=2$, so the maximal subspace of $P$ that lies in the closure of $F_{i}$ and $E-F_{i}$ has rank two. The points $a_{i}, b_{i}$, and $c_{i}$ are all parallel to points in this subspace.

Define $N^{+}$to be the matroid consisting of the points in $E(N)$ and $\cup_{i \in I}\left\{a_{i}, b_{i}, c_{i}\right\}$. (We have defined $N^{+}$relative to a representation of $N$. In fact, any two representations of $N$ will lead to the same matroid $N^{+}$, but we will not make use of this fact.) We define $\operatorname{Core}(N)$ to be the matroid obtained from $N^{+}$by deleting $\cup_{i \in I} F_{i}$ and any other point of $E(N)$ that is parallel to a point $a_{i}$ or $c_{i}$ for some $i \in I$. Figure 3 shows schematic representations of the matroids $N, N^{+}$, and $\operatorname{Core}(N)$. Proposition 3.4 shows that $\mathcal{T}=$ $\left\{\left\{a_{i}, b_{i}, c_{i}\right\}\right\}_{i \in I}$ is a family of pairwise disjoint triangles in $\operatorname{Core}(N)$. We say that $\operatorname{Core}(N)$ and $\mathcal{T}$ are determined by $\mathcal{F}$.

If $F_{i}=\left(e_{1}, \ldots, e_{n}\right)$ is a fan in $\mathcal{F}$, then we define $F_{i}^{+}$to be the sequence $\left(x_{1}, \ldots, x_{m}\right)$, formed from $\left(e_{1}, \ldots, e_{n}\right)$ by prepending $a_{i}$ if $e_{1}$ is a rim element, and appending $c_{i}$ if $e_{n}$ is a rim element. Note that if $e_{1}$ is a rim element, then orthogonality and our choice of $a_{i}$ mean that $a_{i}, e_{1}$, and $e_{2}$ are pairwise distinct. Thus $\left\{x_{1}, x_{2}, x_{3}\right\}$ is a triangle of $N^{+}$in any case. Similarly, $\left\{x_{m-2}, x_{m-1}, x_{m}\right\}$ is a triangle of $N^{+}$. Any triangle of $N$ contained 

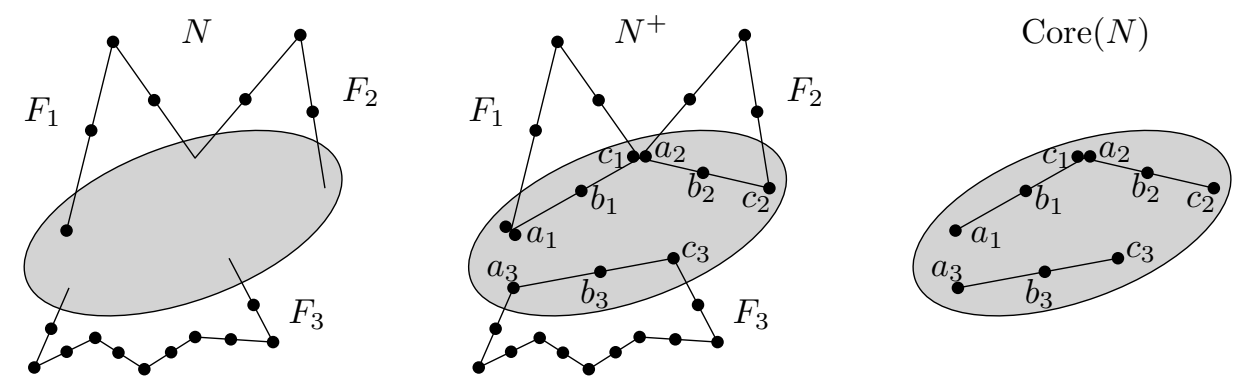

Figure 3: Geometric illustrations of $N, N^{+}$, and Core $(N)$.

in $F_{i}$ is also a triangle in $N^{+}$. If $T^{*}$ is a triad of $N$ contained in $F_{i}$, then every point in $\cup_{i \in I}\left\{a_{i}, b_{i}, c_{i}\right\}$ is in the closure of the complement of $T^{*}$. Therefore $T^{*}$ is a triad in $N^{+}$. We have just shown that $F_{i}^{+}$is a fan in $N^{+}$, and that both $x_{1}$ and $x_{m}$ are spoke elements. This implies that $m$ is odd.

Proposition 3.4. For each $i \in I,\left\{a_{i}, b_{i}, c_{i}\right\}$ is a triangle in $N^{+}$.

Proof. We have already noted that $r_{N^{+}}\left(\left\{a_{i}, b_{i}, c_{i}\right\}\right) \leqslant 2$. Let $F_{i}=\left(e_{1}, \ldots, e_{n}\right)$, and let $F_{i}^{+}$be $\left(x_{1}, \ldots, x_{m}\right)$. Assume that $a_{i}$ and $c_{i}$ are parallel. This means that $x_{1}$ and $x_{m}$ are parallel. As $\left\{x_{1}, x_{2}, x_{3}\right\}$ and $\left\{x_{m-2}, x_{m-1}, x_{m}\right\}$ are triangles, there is a circuit contained in $\left\{x_{2}, x_{3}, x_{m-2}, x_{m-1}\right\}$ that contains $x_{m-1}$. From this it follows that if $x_{m-1}=e_{k}$ (where $k$ is either $n-1$ or $n)$, then $e_{k}$ is in the closure of $\left\{e_{1}, \ldots, e_{k-1}\right\}$. However, we also know that $\left\{e_{k-2}, e_{k-1}, e_{k}\right\}$ is a triad, so $e_{k}$ is in the coclosure of $\left\{e_{1}, \ldots, e_{k-1}\right\}$ in $N$. From this we can deduce that $\lambda_{N}\left(\left\{e_{1}, \ldots, e_{k}\right\}\right) \leqslant 1$, and this leads to a contradiction to the fact that $N$ is 3 -connected. Therefore $a_{i}$ and $c_{i}$ are not parallel. Next we will show that $a_{i}$ is not parallel to $b_{i}$. A symmetrical argument can be used to prove that $c_{i}$ is not parallel to $b_{i}$, and that therefore $\left\{a_{i}, b_{i}, c_{i}\right\}$ is a triangle, as desired. Assume that $a_{i}$ and $b_{i}$ are parallel. The set of rim elements in $\left(e_{1}, \ldots, e_{n}\right)$ is equal to $\left\{x_{2}, x_{4}, \ldots, x_{m-1}\right\}$. Thus $a_{i}$ is in the closure of this set and of $\left\{x_{2}, x_{3}\right\}$. Hence $\left\{x_{2}, x_{3}, x_{4}, x_{6}, \ldots, x_{m-1}\right\}$ contains a circuit, and this circuit must contain at least three elements. If $k$ is the largest integer such that $e_{k}$ is in this circuit, then $e_{k}$ is in the closure and the coclosure of $\left\{e_{1}, \ldots, e_{k-1}\right\}$ in $N$. From this we can derive a contradiction to the 3-connectivity of $N$.

If $F_{1}=\left(e_{1}, \ldots, e_{m}\right)$ and $F_{2}=\left(e_{1}^{\prime}, \ldots, e_{n}^{\prime}\right)$ are fans, we will say that $F_{1}$ is enclosed in $F_{2}$ if either $\left(e_{1}, \ldots, e_{m}\right)$ or $\left(e_{m}, \ldots, e_{1}\right)$ is equal to $\left(e_{i}^{\prime}, \ldots, e_{i+m-1}^{\prime}\right)$, for some $i \in\{1, \ldots, n-$ $m+1\}$. Let $\mathcal{F}$ and $\mathcal{F}^{\prime}$ be collections of fans. We will say that $\mathcal{F}$ is enclosed in $\mathcal{F}^{\prime}$ if there is an bijective function from $\mathcal{F}$ to $\mathcal{F}^{\prime}$ such that every fan in $\mathcal{F}$ is enclosed in its image.

The next result shows that a fan-extension of $N$ can be constructed by gluing wheels to Core $(N)$, where Core $(N)$ is defined relative to some pairwise disjoint family of fans in $N$. Note that Corollary 2.5 and the hypotheses of the lemma imply that $M$ is a fan-extension of $N$.

Lemma 3.5. Let $N$ be a 3-connected representable matroid, where $|E(N)| \geqslant 4$ and $N$ is neither a wheel nor a whirl. Let $\mathcal{F}_{N}$ be a pairwise disjoint family of fans in $N$. Assume 
there is no fan, $F$, in $N$ such that two distinct fans in $\mathcal{F}_{N}$ (considered as unordered sets) are contained in $F$. Assume that $M$ is a 3-connected matroid with $N$ as a minor, and every minor of $M$ with $N$ as a minor is 3-connected up to series and parallel sets. Let $\mathcal{F}$ be a covering family in $M$ relative to $\mathcal{F}_{N}$. There exists a pairwise disjoint family of fans, $\mathcal{F}_{0}$, in $N$, such that $\mathcal{F}_{0}$ encloses $\mathcal{F}_{N}$, and moreover, up to relabeling, $M=\otimes(\operatorname{Core}(N), \mathcal{T}, r, X)$, for some blueprint $(\operatorname{Core}(N), \mathcal{T}, r, X)$ where $\operatorname{Core}(N)$ and $\mathcal{T}$ are determined by $\mathcal{F}_{0}$. Furthermore, $\mathcal{F}$ is enclosed in the family of canonical fans of $\otimes(\operatorname{Core}(N), \mathcal{T}, r, X)$.

Proof. Let $E=E(N)$. The proof will be by induction on $|E(M)|-|E|$. First we assume $|E(M)|=|E|$. Then $M=N$. Now $\mathcal{F}$ is a covering family of $N$, relative to $\mathcal{F}_{N}$. This does not mean that $\mathcal{F}=\mathcal{F}_{N}$. The fans in $\mathcal{F}_{N}$ may be enclosed in larger fans, and these larger fans may be members of $\mathcal{F}$. Nonetheless, $\mathcal{F}$ is a pairwise disjoint family of fans in $N$. Because each fan in $\mathcal{F}_{N}$ is consistent with a fan in $\mathcal{F}$, it follows without difficulty from Lemma 5.8 that each fan in $\mathcal{F}_{N}$ must be enclosed by a fan in $\mathcal{F}$. Thus we can define $\mathcal{F}_{0}$ to be $\mathcal{F}$. Let $\mathcal{F}=\left\{F_{i}\right\}_{i \in I}$, where $I=\{1, \ldots, t\}$, and let Core $(N)$ and $\mathcal{T}=\left\{\left\{a_{i}, b_{i}, c_{i}\right\}\right\}_{i \in I}$ be the matroid and family of triangles determined by $\mathcal{F}$. To prove the base case of the induction, we now need only check that $N=\otimes(\operatorname{Core}(N), \mathcal{T}, r, X)$ (up to relabeling).

Recall that $N^{+}$is the matroid consisting of the points in $E$ and $\cup_{i \in I}\left\{a_{i}, b_{i}, c_{i}\right\}$. Let $F_{t}^{+}$be $\left(x_{1}, \ldots, x_{m}\right)$. This is a fan in $N^{+}$, and $x_{1}$ and $x_{m}$ are spoke elements. The rim elements in $F_{t}$ are $\left\{x_{2}, x_{4}, \ldots, x_{m-1}\right\}$. Thus $b_{t}$ is in the closure of this set. Any circuit contained in $\left\{x_{2}, x_{4}, \ldots, x_{m-1}, b_{t}\right\}$ must contain $\left\{x_{2}, x_{4}, \ldots, x_{m-1}\right\}$, for otherwise we can find a violation of orthogonality with one of the triads in $F_{t}$. Thus $\left\{x_{2}, x_{4}, \ldots, x_{m-1}, b_{t}\right\}$ is a circuit in $N^{+}$, so $\left\{x_{m-1}, b_{t}\right\}$ is a circuit in $N^{+} /\left\{x_{2}, x_{4}, \ldots, x_{m-3}\right\} \backslash\left\{x_{3}, x_{5}, \ldots, x_{m-2}\right\}$. We are going to apply a result by Oxley and $\mathrm{Wu}[10$, Theorem 1.8]. This theorem applies only to 3-connected matroids, but the simplification of $\mathrm{N}^{+}$is 3-connected, and it is easy to see that the result still holds. The theorem by Oxley and $\mathrm{Wu}$ tells us that, up to relabeling, $N^{+}$is equal to $\left(N^{+} \backslash F_{t}\right) \bigotimes_{\left\{a_{t}, b_{t}, c_{t}\right\}} W_{t}$, where $W_{t}$ has rank $(m+1) / 2$. Moreover $N^{+} \backslash F_{t}$ is 3 -connected up to parallel pairs. Now $F_{t-1}^{+}$is a fan in $N^{+}$. Note that $F_{t}$ is disjoint from $F_{t-1}^{+}$by construction of $\operatorname{Core}(N)$. As $\operatorname{si}\left(N^{+} \backslash F_{t}\right)$ is 3-connected, it is easy to see that $F_{t-1}^{+}$is a fan in $N^{+} \backslash F_{t}$ also. By again using [10, Theorem 1.8], we see that $N^{+} \backslash F_{t}$ can be obtained by using generalized parallel connection to glue a wheel of the correct rank to $N^{+} \backslash F_{t} \backslash F_{t-1}$ along the line $\left\{a_{t-1}, b_{t-1}, c_{t-1}\right\}$. We proceed inductively in this way, and conclude that $N^{+}$is obtained from

$$
N^{+} \backslash F_{t} \backslash F_{t-1} \backslash \cdots \backslash F_{1}
$$

by attaching wheels via generalized parallel connections. Let $S$ be the set of elements in $E-\left(\cup_{i \in I} F_{i}\right)$ that are parallel to some element $a_{i}$ or $c_{i}$. Then Core $(N)=N^{+} \backslash\left(\cup_{i \in I} F_{i}\right) \backslash S$. This means that $N^{+} \backslash S$ can be obtained from $\operatorname{Core}(N)$ by attaching wheels to the lines $\left\{\left\{a_{i}, b_{i}, c_{i}\right\}\right\}_{i \in I}$. This in turn means that $N$ can be obtained from Core $(N)$ in the following way: attach wheels to the lines $\left\{\left\{a_{i}, b_{i}, c_{i}\right\}\right\}_{i \in I}$, and then, for each element $s \in S$, distinguish an element in $\left\{\left\{a_{i}, b_{i}, c_{i}\right\}\right\}_{i \in I}$ that is parallel to it, and relabel that element as $s$, and then finally delete all other elements in $\left\{\left\{a_{i}, b_{i}, c_{i}\right\}\right\}_{i \in I}$. In other words, up to relabeling, we can obtain $N$ from $\operatorname{Core}(N)$ by gluing on wheels. This is exactly what we aimed to prove, and it establishes the base case of our induction. 
Now we can assume that $M \neq N$. Proposition 2.4 implies that there is a fan $F \in \mathcal{F}$ such that $M$ and $F$ are obtained from a 3-connected matroid $M^{\prime}$ by performing a fanlengthening move on the fan $F^{\prime}$. It is clear that $\mathcal{F}^{\prime}=(\mathcal{F}-\{F\}) \cup\left\{F^{\prime}\right\}$ is a covering family in $M^{\prime}$, and we can apply the inductive hypothesis. There is a family, $\mathcal{F}_{0}$, of pairwise disjoint fans in $N$ such that $\mathcal{F}_{0}$ encloses $\mathcal{F}_{N}$. We relabel $M^{\prime}$ in such a way that it is obtained by gluing wheels to $\operatorname{Core}(N)$ (relative to $\mathcal{F}_{0}$ ), and $\mathcal{F}^{\prime}$ is enclosed in the family of canonical fans. Let $X$ be the set of elements that we delete after attaching wheels to $\operatorname{Core}(N)$. This means that $F^{\prime}$ is enclosed in the canonical fan $\left(x_{1}^{i}, y_{1}^{i}, x_{2}^{i}, \ldots, y_{r(i)-1}^{i}, x_{r(i)}^{i}\right)-X$, for some $i$. Let $F$ be $\left(e_{1}, \ldots, e_{m}\right)$.

Assume that $M^{\prime}$ is obtained from $M$ by deleting a terminal spoke element of $F$. By reversing, we may assume that $M^{\prime}=M \backslash e_{1}$. Now $F^{\prime}=\left(e_{2}, \ldots, e_{m}\right)$ is enclosed in $\left(x_{1}^{i}, y_{1}^{i}, x_{2}^{i}, \ldots, y_{r(i)-1}^{i}, x_{r(i)}^{i}\right)-X$. As $\left\{e_{2}, e_{3}, e_{4}\right\}$ is a triad in $M^{\prime}$, by reversing $\left(x_{1}^{i}, y_{1}^{i}, x_{2}^{i}, \ldots, y_{r(i)-1}^{i}, x_{r(i)}^{i}\right)$ as necessary, we can assume $e_{2}=y_{k}^{i}$ and $e_{3}=x_{k+1}^{i}$. Assume for a contradiction that either (i) $k>1$, or (ii) $k=1$ and $x_{1}^{i}=e_{1}$, and hence $x_{1}^{i} \in E(N)$, or (iii) $k=1$ and $x_{1}^{i}=a_{i}$, but $a_{i} \notin X$. If any of these situations hold, then $\left\{x_{k}^{i}, y_{k}^{i}, x_{k+1}^{i}\right\}$ and $\left\{e_{1}, e_{2}, e_{3}\right\}$ are both triangles in $M$, and they intersect in the elements $e_{2}$ and $e_{3}$. This means that $\left\{x_{k}^{i}, e_{1}, e_{2}\right\}$ is a triangle in $M$ that intersects the triad $\left\{e_{2}, e_{3}, e_{4}\right\}$ in a single element. This contradiction means that $k=1, x_{1}^{i}=a_{i}$, and $a_{i} \in X$. Now let $M^{\prime \prime}$ be the matroid obtained by gluing the same wheels to $\operatorname{Core}(N)$, except that instead of deleting $X$, we delete $X-x_{1}^{i}$. Obviously $M^{\prime \prime} \backslash x_{1}^{i}=M^{\prime}=M \backslash e_{1}$. We relabel $M$ so that it inherits the relabeling of $M^{\prime}$ and $e_{1}$ is relabeled as $x_{1}^{i}$. This means that $\left(x_{1}^{i}, y_{1}^{i}, x_{2}^{i}, y_{2}^{i}\right)$ is a fan in both $M$ and $M^{\prime \prime}$. Now Proposition 3.1 implies that $M^{\prime \prime}=M$. It is clear that after the relabeling $F$ is enclosed in the canonical fan $\left(x_{1}^{i}, y_{1}^{i}, x_{2}^{i}, \ldots, y_{r(i)-1}^{i}, x_{r(i)}^{i}\right)-\left(X-x_{1}^{i}\right)$, so the result holds.

Next we will assume that $M^{\prime}=M / e_{1}$, where $e_{1}$ is a rim element in $F$. We can assume that $e_{2}=x_{k}^{i}$ and $e_{3}=y_{k}^{i}$. If $k>1$, then $\left\{y_{k-1}^{i}, x_{k}^{i}, y_{k}^{i}\right\}$ is a triad in $M^{\prime}$, and hence in $M$. As $\left\{e_{1}, e_{2}, e_{3}\right\}$ is also a triad of $M$, it follows that $\left\{y_{k-1}^{i}, e_{1}, e_{2}\right\}$ is a triad in $M$. As $\left\{e_{2}, e_{3}, e_{4}\right\}$ is a triangle, this is a contradiction, so $k=1$. Therefore $a_{i}$ is parallel to $x_{1}^{i}=e_{2}$. This means that we can assume that $e_{2}$ is relabeled as $a_{i}$ when $M^{\prime}$ is relabeled in such a way that it becomes equal to $\otimes(\operatorname{Core}(N), \mathcal{T}, r, X)$. Thus $a_{i} \notin X$. Let $W_{i}^{\prime \prime}$ be a copy of a wheel with rank $r(i)+1$. Let the ground set of $W_{i}^{\prime \prime}$ be $E\left(W_{i}\right) \cup\{x, y\}$, labeled in such a way that $\left(x, y, x_{1}^{i}, y_{1}^{i}, x_{2}^{i}, \ldots, y_{r(i)-1}^{i}, x_{r(i)}^{i}, y_{r(i)}^{i}\right)$ is a fan and $\left\{x, y_{r(i)}^{i}, x_{r(i)}^{i}\right\}$ is a triangle. Therefore $x$ is identified with $a_{i}$. Now let $M^{\prime \prime}$ be the matroid obtained from $\operatorname{Core}(N)$ by gluing on the same wheels as before, except that we use $W_{i}^{\prime \prime}$ instead of $W_{i}$, and we then delete $X \cup a_{i}$. Now $W_{i}^{\prime \prime} / y \backslash x=W_{i}$. From this it follows easily that $M^{\prime \prime} / y=M^{\prime}$. We relabel $M$ in such a way that it inherits the labeling we applied to $M^{\prime}$, and $e_{1}$ is labeled as $y$. Now $\left(y, x_{1}^{i}, y_{1}^{i}, x_{2}^{i}\right)$ is a fan in both $M$ and $M^{\prime \prime}$, and $M / y=M^{\prime}=M^{\prime \prime} / y$. Therefore the dual of Proposition 3.1 implies that $M^{\prime \prime}=M$, as desired.

For the final case, we assume $\left|E\left(M^{\prime}\right)\right|=|E(M)|-2$. Since $(\mathcal{F}-\{F\}) \cup\left\{F^{\prime}\right\}$ is a covering family in $M^{\prime}$, it follows that $m \geqslant 5$. By reversing $F$ as necessary, we can assume that $M^{\prime}=M / e_{j} \backslash e_{j+1}$, where $e_{j}$ is a rim element of $F$. We will assume that $j>1$. An almost identical argument will hold in the case that $j=1$. Now $\left(e_{1}, \ldots, e_{j-1}, e_{j+2}, \ldots, e_{m}\right)$ is a fan of $M^{\prime}$ that is enclosed in $\left(x_{1}^{i}, y_{1}^{i}, x_{2}^{i}, \ldots, y_{r(i)-1}^{i}, x_{r(i)}^{i}\right)$. 
By reversing $\left(x_{1}^{i}, y_{1}^{i}, x_{2}^{i}, \ldots, y_{r(i)-1}^{i}, x_{r(i)}^{i}\right)$ as necessary, we can assume that $e_{j-1}=x_{k}^{i}$ and $e_{j+2}=y_{k}^{i}$ (if $j+2 \leqslant m$ ). Let $W_{i}^{\prime \prime}$ be a copy of a wheel with $\operatorname{rank} r(i)+1$, and let the ground set of $W_{i}^{\prime \prime}$ be $E\left(W_{i}\right) \cup\{x, y\}$. We label $W_{i}^{\prime \prime}$ in such a way that $\left(x_{1}^{i}, y_{1}^{i}, \ldots, x_{k}^{i}, y, x, y_{k}^{i}, \ldots, x_{r(i)}^{i}\right)$ is a fan. Now let $M^{\prime \prime}$ be the matroid obtained from $\operatorname{Core}(N)$ by gluing on the same wheels used to obtain $M^{\prime}$, except that we use $W_{i}^{\prime \prime}$ instead of $W_{i}$. After attaching these wheels to $\operatorname{Core}(N)$ we delete exactly the same set $X$. Now $W_{i}^{\prime \prime} / y \backslash x=W_{i}$, and it follows that $M^{\prime \prime} / y \backslash x=M^{\prime}$. We relabel $M$ so that it inherits the relabeling of $M^{\prime}$, while $e_{i}$ is labeled as $y$ and $e_{i+1}$ is labeled as $x$. After this relabeling, $M / y \backslash x=M^{\prime}=M^{\prime \prime} / y \backslash x$. We claim that this implies $M=M^{\prime \prime}$. Note that $M / y$ and $M^{\prime \prime} / y$ are both obtained by adding $x$ parallel to $x_{k}^{i}$ in $M / y \backslash x=M^{\prime \prime} / y \backslash x$. Thus $M / y=M^{\prime \prime} / y$. If either $\left(x_{k-1}^{i}, y_{k-1}^{i}, x_{k}^{i}, y\right)$ or $\left(y, x, y_{k}^{i}, x_{k+1}^{i}\right)$ is a fan in both $M$ and $M^{\prime \prime}$, then the dual of Proposition 3.1 implies that $M=M^{\prime \prime}$ as desired. If neither of these sequences is a fan in $M$ and $M^{\prime \prime}$, then since $m \geqslant 5$, it is not difficult to see that $m=5$, and $e_{j}=e_{3}$. Thus $\left(x_{1}^{i}, x_{2}^{i}, y, x, x_{3}^{i}\right)$ is a fan in both $M$ and $M^{\prime \prime}$. In this case $M \backslash x$ and $M^{\prime \prime} \backslash x$ are both obtained from $M / y \backslash x=M^{\prime \prime} / y \backslash x$ by adding $y$ in series to $x_{3}^{i}$. Thus $M \backslash x=M^{\prime \prime} \backslash x$, and we can use Proposition 3.1 to show that $M=M^{\prime \prime}$, as claimed. Obviously $F$ is contained in the canonical fan associated with gluing $W_{i}^{\prime \prime}$ to $\operatorname{Core}(N)$, so the proof is complete.

\section{Applications}

Now we consider some applications of Theorem 1.2. We omit the proofs, since Theorem 1.2 and Lemma 3.5 reduce them to computational case checking. Details can be found in [1]. Our first application concerns the binary matroids that are $\left\{F_{7}, F_{7}^{*}\right\}$-fragile. We describe these via grafts (see [9, p. 386]). Recall that $N_{12}$ is illustrated in Figure 1(i). Figure 4 gives graft representations of the matroids $N_{11}^{+}$and $N_{12}$. In both case, the distinguished vertices in the graft are those vertices with squares around them. In $N_{12}$, let $w_{4}$ be the matroid element that corresponds to the set of distinguished vertices.
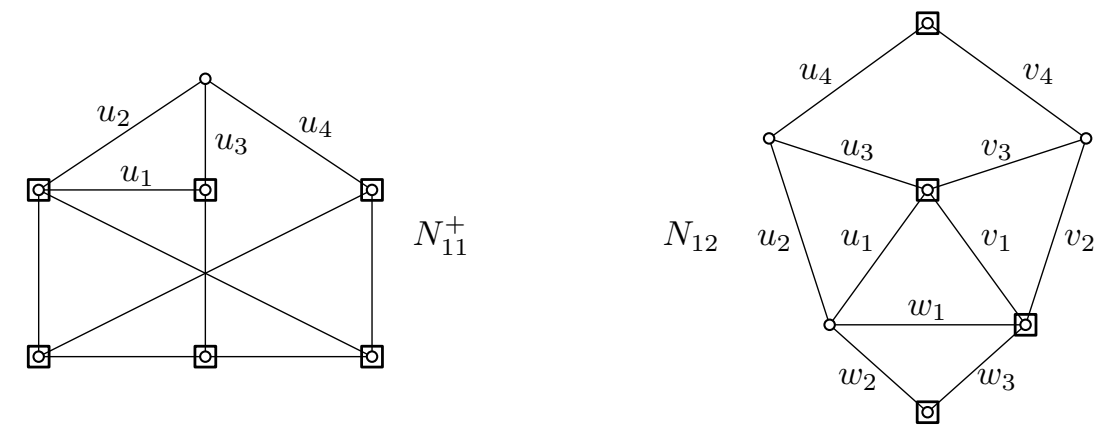

Figure 4: Graft representations of $N_{11}^{+}$and $N_{12}$.

Now we have the tools to characterize binary $\left\{F_{7}, F_{7}^{*}\right\}$-fragile matroids that have $N_{11}^{+}$ or $N_{12}$ as a minor. The following result is known to Truemper [13] and Kingan and Lemos [6]. It is a straightforward application of Theorem 1.2 and Lemma 3.5. 
Theorem 4.1. Let $M$ be a 3-connected binary $\left\{F_{7}, F_{7}^{*}\right\}$-fragile matroid. If $M$ has $N_{11}^{+}$as a minor, then $M$ is a fan-extension of $N_{11}^{+}$relative to $\left\{\left(u_{1}, u_{2}, u_{3}, u_{4}\right)\right\}$, and $M$ is a restriction of a member of the family illustrated in the lefthand diagram in Figure 5. If $M$ has $N_{12}$ as a minor, then $M$ is a fan-extension of $N_{12}$ relative to $\left\{\left(u_{1}, u_{2}, u_{3}, u_{4}\right),\left(v_{1}, v_{2}, v_{3}, v_{4}\right),\left(w_{1}, w_{2}, w_{3}, w_{4}\right)\right\}$, and $M$ is a restriction of a member of the family illustrated in the righthand diagram in Figure 5.
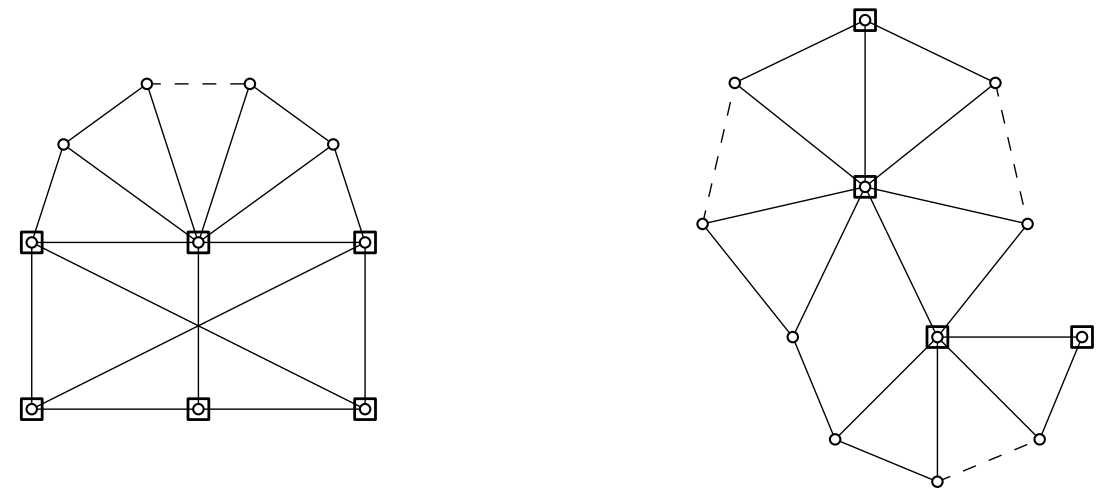

Figure 5: The $N_{11}^{+}$and $N_{12}$ families.

Next we consider fragile classes of matroid representable over the partial fields $\mathbb{U}_{2}$ and $\mathbb{H}_{5}$. Every $\mathbb{U}_{2}$-representable matroid is also $\mathbb{H}_{5}$-representable, so it suffices to consider only the latter partial field. We currently have a solid understanding of the $\mathbb{H}_{5}$-representable $\left\{U_{2,5}, U_{3,5}\right\}$-fragile matroids, and this brings excluded-minor characterizations for these two partial fields within grasp. Proofs and definitions can all be found in [1].

Proposition 4.2. Let $M$ be a 3 -connected $\mathbb{H}_{5}$-representable $\left\{U_{2,5}, U_{3,5}\right\}$-fragile matroid. Assume that $M$ has $N$ as minor, where $N$ is in $\left\{M_{9,0}, M_{9,2}, M_{9,15}, M_{9,18}\right\}$. Then $M$ is a fan-extension of $N$.

Theorem 4.3. Let $M$ be a 3-connected $\mathbb{H}_{5}$-representable matroid. Up to relabeling, one of the following statements holds:

(i) $M$ has one of $X_{8}, Y_{8}$, or $Y_{8}^{*}$ as a minor,

(ii) $M$ is in $\left\{U_{2,6}, U_{3,6}, U_{4,6}, P_{6}, M_{9,9}, M_{9,9}^{*}\right\}$,

(iii) $M$ or $M^{*}$ can be obtained from $U_{2,5}$ (with groundset $\{a, b, c, d, e\}$ ) by gluing wheels to $\{(a, c, b),(a, d, b),(a, e, b)\}$,

(iv) $M$ or $M^{*}$ can be obtained from $U_{2,5}$ (with groundset $\{a, b, c, d, e\}$ ) by gluing wheels to $\{(a, b, c),(c, d, e)\}$,

(v) $M$ or $M^{*}$ can be obtained from $M_{7,1}$ by gluing on a wheel. 


\section{$5 \quad$ Results on fans}

Now we start moving towards a proof of our main theorem. This section collects some results that we will need. The next proposition can be proved with an easy inductive argument.

Proposition 5.1. Let $\left(e_{1}, \ldots, e_{n}\right)$ be a fan of the matroid $M$. Then $\left\{e_{1}, \ldots, e_{n}\right\}$ is 3-separating in $M$.

Proposition 5.2. Let $M$ be a 3-connected matroid, and let $F_{1}=\left(e_{1}, \ldots, e_{n}\right)$ be a fan of $M$ such that $n \geqslant 5$. Let $e_{i}$ be a rim element, for some $i \in\{1, \ldots, n-1\}$. Then $F_{2}=\left(e_{1}, \ldots, e_{i-1}, e_{i+2}, \ldots, e_{n}\right)$ is a fan of $M / e_{i} \backslash e_{i+1}$, and for all $j, e_{j}$ is a spoke element in $F_{1}$ if and only if it is a spoke element in $F_{2}$

Proof. Any triad of $M$ contained in $\left\{e_{1}, \ldots, e_{i-2}\right\}$ remains a triad in $M / e_{i} \backslash e_{i+1}$, by orthogonality with the triangle $\left\{e_{i-1}, e_{i}, e_{i+1}\right\}$. Similarly, any triad of $M$ in $\left\{e_{i+4}, \ldots, e_{n}\right\}$ is a triad in $M / e_{i} \backslash e_{i+1}$ by orthogonality with $\left\{e_{i+1}, e_{i+2}, e_{i+3}\right\}$. In the same way, the triad $\left\{e_{i}, e_{i+1}, e_{i+2}\right\}$ implies that any triangle in $\left\{e_{1}, \ldots, e_{i-1}\right\}$ or $\left\{e_{i+3}, \ldots, e_{n}\right\}$ is also a triangle in $M / e_{i} \backslash e_{i+1}$.

Now the only sets we need check are $\left\{e_{i-2}, e_{i-1}, e_{i+2}\right\}$ and $\left\{e_{i-1}, e_{i+2}, e_{i+3}\right\}$. Strong cocircuit-exchange between $\left\{e_{i-2}, e_{i-1}, e_{i}\right\}$ and $\left\{e_{i}, e_{i+1}, e_{i+2}\right\}$ gives a cocircuit contained in $\left\{e_{i-2}, e_{i-1}, e_{i+1}, e_{i+2}\right\}$ that contains $e_{i+1}$. This cocircuit contains $e_{i-1}$, by orthogonality with $\left\{e_{i-1}, e_{i}, e_{i+1}\right\}$. If $e_{i-2}$ is not in the cocircuit, then $\left\{e_{i-1}, e_{i+1}, e_{i+2}\right\}$ and $\left\{e_{i}, e_{i+1}, e_{i+2}\right\}$ are triads, so $\left\{e_{i-1}, e_{i}, e_{i+1}\right\}$ is a triad and a triangle. This contradicts the fact that $M$ is 3 -connected with at least 5 elements. Hence $e_{i-2}$ is in the cocircuit, and a similar argument shows that $\left\{e_{i-2}, e_{i-1}, e_{i+1}, e_{i+2}\right\}$ is a cocircuit of $M$. Thus $\left\{e_{i-2}, e_{i-1}, e_{i+2}\right\}$ is a triad in $M / e_{i} \backslash e_{i+1}$, as desired. We can show that $\left\{e_{i-1}, e_{i+2}, e_{i+3}\right\}$ is a triangle of $M / e_{i} \backslash e_{i+1}$ by a dual argument.

Proposition 5.3. Let $\left(e_{1}, \ldots, e_{n}\right)$ be a fan in the 3 -connected matroid $M$, and assume that $n \geqslant 4$. Let $e \in E(M)-\left\{e_{1}, \ldots, e_{n}\right\}$ be such that there is a triangle, $T$, satisfying $\{e\} \subseteq T \subseteq\left\{e, e_{1}, \ldots, e_{n}\right\}$. Then either:

(i) $T=\left\{e, e_{1}, e_{2}\right\}, e_{1}$ is a rim element, and $\left(e, e_{1}, \ldots, e_{n}\right)$ is a fan,

(ii) $T=\left\{e, e_{n-1}, e_{n}\right\}, e_{n}$ is a rim element, and $\left(e_{1}, \ldots, e_{n}, e\right)$ is a fan,

(iii) $T=\left\{e, e_{1}, e_{n}\right\}$, and $e_{1}$ and $e_{n}$ are spoke elements,

(iv) $T=\left\{e, e_{2}, e_{4}\right\}, e_{2}$ is a rim element, and $n \leqslant 5$, or

(v) $T=\left\{e, e_{n-1}, e_{n-3}\right\}, e_{n-1}$ is a rim element, and $n \leqslant 5$.

Proof. Let $T=\left\{e, e_{x}, e_{y}\right\}$, where $1 \leqslant x<y \leqslant n$. If $x=1$ and $y=n$, then statement (iii) must hold, since orthogonality with $T$ requires that $\left\{e_{1}, e_{2}, e_{3}\right\}$ and $\left\{e_{n-2}, e_{n-1}, e_{n}\right\}$ are both triangles. We will assume that $x>1$, and show that statement (ii) or statement (iv) holds. If $x=1$, then $y<n$, so we can replace $\left(e_{1}, \ldots, e_{n}\right)$ with $\left(e_{n}, \ldots, e_{1}\right)$, and swap 
labels on $x$ and $y$, and then apply exactly the same arguments to deduce that (i) or (v) holds.

Now we assume that $x>1$. Assume also that $y=x+1$. If $y<n$, then either $\left\{e_{x-1}, e_{x}, e_{x+1}\right\}$ is a triangle and $\left\{e_{x}, e_{x+1}, e_{x+2}\right\}$ is a triad, or $\left\{e_{x}, e_{x+1}, e_{x+2}\right\}$ is a triangle and $\left\{e_{x-1}, e_{x}, e_{x+1}\right\}$ is a triad. As $M$ is 3 -connected, in the first case $\left\{e, e_{x-1}, e_{x}\right\}$ is a triangle, and in the second case $\left\{e, e_{x+1}, e_{x+2}\right\}$ is a triangle. In either case we have a contradiction to orthogonality. Therefore $y=n$, and $e_{y}$ is a rim element, by orthogonality between $T=\left\{e, e_{n-1}, e_{n}\right\}$ and $\left\{e_{n-3}, e_{n-2}, e_{n-1}\right\}$. Now it is clear that statement (ii) holds. Hence we will assume that $y>x+1$. Thus $e_{x}$ is a rim element, by orthogonality between $T$ and $\left\{e_{x-1}, e_{x}, e_{x+1}\right\}$. If $2<x$, then we have a contradiction to orthogonality between $T$ and $\left\{e_{x-2}, e_{x-1}, e_{x}\right\}$. Therefore $x=2$. If $y>x+2$, then $T$ intersects the triad $\left\{e_{x}, e_{x+1}, e_{x+2}\right\}$ in a single element. Therefore $y=x+2=4$. Finally, $n \leqslant 5$, for otherwise $T$ intersects the triad $\left\{e_{y}, e_{y+1}, e_{y+2}\right\}$ in a single element. We have shown that statement (iv) holds, so the proof is complete.

Proposition 5.4. Assume that $\left(e_{1}, \ldots, e_{n}\right)$ is a fan of the 3 -connected matroid $M$, and that $4 \leqslant|E(M)| \leqslant n+1$. Then $M$ is a wheel or a whirl.

Proof. If $|E(M)|=4$, then $M$ is isomorphic to the whirl $U_{2,4}$, and we are done. Therefore we assume $|E(M)|>4$. Since wheels and whirls are self-dual, we can switch to $M^{*}$ as required, and assume that $e_{1}$ is a spoke element of $\left(e_{1}, \ldots, e_{n}\right)$. If $|E(M)|=n$, then result follows immediately from [10, Lemma 4.8]. Therefore we assume that $|E(M)|=n+1$. Let $e$ be the single element in $E(M)-\left\{e_{1}, \ldots, e_{n}\right\}$.

Suppose that $e_{n}$ is a rim element, so that $n$ is even. Then $\left\{e_{1}, e_{3}, \ldots, e_{n-3}, e_{n-1}\right\}$ spans $E(M)-\left\{e, e_{n}\right\}$. Since $M$ is 3 -connected, it also spans $E(M)$. Let $C$ be a circuit contained in $\left\{e_{1}, e_{3}, \ldots, e_{n-3}, e_{n-1}\right\} \cup\{e\}$ that contains $e$. If $i$ is odd and $3 \leqslant i \leqslant n-1$, then $e_{i} \notin C$, or else $C$ intersects the triad $\left\{e_{i-1}, e_{i}, e_{i+1}\right\}$ in a single element. Therefore $C \subseteq\left\{e, e_{1}\right\}$, which is impossible as $M$ is 3-connected. This implies that $e_{n}$ is a spoke element and $n$ is odd. Suppose that $\left\{e_{3}, e_{5}, \ldots, e_{n-2}, e_{n}\right\}$ spans $E(M)$. Let $C$ be a circuit in $\left\{e_{3}, e_{5}, \ldots, e_{n-2}, e_{n}\right\} \cup\left\{e_{1}\right\}$ that contains $e_{1}$. Then $C \subseteq\left\{e_{1}, e_{n}\right\}$, for otherwise $C$ intersects a triad in a single element. This is a contradiction, so $\left\{e_{3}, e_{5}, \ldots, e_{n-2}, e_{n}\right\}$ does not span $E(M)$. Since it spans $E(M)-\left\{e, e_{1}, e_{2}\right\}$, it follows that $\left\{e, e_{1}, e_{2}\right\}$ is a triad. Thus $\left(e, e_{1}, \ldots, e_{n}\right)$ is a fan, and we can again apply [10, Lemma 4.8] to deduce that $M$ is a wheel or a whirl.

Proposition 5.5. Let $M$ be a 3-connected matroid with $|E(M)| \geqslant 4$. Let $\left(e_{1}, \ldots, e_{n}\right)$ be a fan of $M$. If there is an element $e \in E(M)-\left\{e_{1}, \ldots, e_{n}\right\}$, and elements $x, y, z \in$ $\left\{e_{1}, \ldots, e_{n}\right\}$ such that $\{e, x, y\}$ is a triangle and $\{e, y, z\}$ is a triad, then $M$ is a wheel or a whirl.

Proof. Let $F=\left\{e_{1}, \ldots, e_{n}\right\}$. Then $\lambda(F) \leqslant 2$, and as $e$ is in the closure and coclosure of $F, \lambda(F \cup e) \leqslant 1$. Therefore the complement of $F \cup e$ contains at most one element. If $n=3$, then either $F$ and $\{e, x, y\}$ are both triangles, or $F$ and $\{e, y, z\}$ are both triads. In the first case $M \mid(F \cup e) \cong U_{2,4}$, and in the second $M^{*} \mid(F \cup e) \cong U_{2,4}$. In either case, 
$F \cup e$ contains a triangle that is a triad. Since $M$ is 3-connected, this means $|E(M)|=4$, and $M$ is isomorphic to $U_{2,4}$, so we are done. Therefore we assume $n \geqslant 4$.

We apply Proposition 5.3. If statement (i) or (ii) holds, then we can apply Proposition 5.4 to $\left(e_{1}, \ldots, e_{n}, e\right)$ or $\left(e, e_{1}, \ldots, e_{n}\right)$ and conclude that $M$ is a wheel or a whirl. Next assume that statement (iii) holds. Then $n \geqslant 5$. By reversing $\left(e_{1}, \ldots, e_{n}\right)$ as necessary, we assume that $y=e_{n}$. Then orthogonality between the triad $\{e, y, z\}$ and the triangle $\left\{e_{n-2}, e_{n-1}, e_{n}\right\}$ requires that $z$ is in $\left\{e_{n-2}, e_{n-1}\right\}$. If $z=e_{n-1}$, then $\left(e_{1}, \ldots, e_{n}, e\right)$ is a fan, and we can again apply Proposition 5.4. Therefore we assume $z=e_{n-2}$. This means that the triangle $\left\{e_{n-4}, e_{n-3}, e_{n-2}\right\}$ and the triad $\{e, y, z\}$ meet in a single element, a contradiction. Therefore statement (iv) or (v) in Proposition 5.3 holds. By reversing, we can assume that $\{x, y\}=\left\{e_{2}, e_{4}\right\}$, and $e_{2}$ is a rim element.

If $n=4$, then we apply Proposition 5.4 to the fan $\left(e_{1}, e_{3}, e_{2}, e_{4}, e\right)$. Therefore we assume $n=5$. If $z=e_{1}$, then $y=e_{2}$, by orthogonality between the triangles $\left\{e_{1}, e_{2}, e_{3}\right\}$ and $\left\{e_{3}, e_{4}, e_{5}\right\}$, and the triad $\{e, y, z\}$. Therefore $\left(e, e_{1}, e_{2}, e_{3}, e_{4}, e_{5}\right)$ is a fan. The same argument disposes of the case that $z=e_{5}$. Therefore we assume $z=e_{3}$. But in this case either $\left\{e_{1}, e_{2}, e_{3}\right\}$ or $\left\{e_{3}, e_{4}, e_{5}\right\}$ is a triangle that intersects the triad $\{e, y, z\}$ in a single element. This contradiction completes the proof.

Proposition 5.6. Let $M$ be a 3-connected matroid such that $|E(M)| \geqslant 4$, and $M$ is not a wheel or a whirl. Let $\left(e_{1}, \ldots, e_{n}\right)$ be a fan of $M$. If $T \subseteq\left\{e_{1}, \ldots, e_{n}\right\}$ is a triangle, then $T=\left\{e_{i}, e_{i+1}, e_{i+2}\right\}$ for some $i \in\{1, \ldots, n-2\}$ such that $e_{i}$ is a spoke element.

Proof. Let $i, j$, and $k$ be such that $1 \leqslant i<j<k \leqslant n$, and $T=\left\{e_{i}, e_{j}, e_{k}\right\}$. If $e_{i}$ is a rim element of $\left(e_{1}, \ldots, e_{n}\right)$, then $\left\{e_{i}, e_{i+1}, e_{i+2}\right\}$ is a triad. Since $T$ is a triangle, it follows that $e_{i}$ is in the closure and the coclosure of $\left\{e_{i+1}, \ldots, e_{n}\right\}$. As $\lambda\left(\left\{e_{i+1}, \ldots, e_{n}\right\}\right) \leqslant 2$, it follows that $\lambda\left(\left\{e_{i}, \ldots, e_{n}\right\}\right) \leqslant 1$. As $M$ is 3 -connected, we deduce that the complement of $\left\{e_{i}, \ldots, e_{n}\right\}$ contains at most one element. Thus $M$ is a wheel or a whirl by Proposition 5.4, and this is a contradiction. Therefore $e_{i}$ is a spoke element. The same argument shows that $e_{k}$ is a spoke element.

Assume that $j>i+1$. If $e_{j}$ is a rim element of $\left(e_{1}, \ldots, e_{n}\right)$, then $j \geqslant i+3$, so $T$ intersects the triad $\left\{e_{j-2}, e_{j-1}, e_{j}\right\}$ in a single element, violating orthogonality. Therefore $e_{j}$ is a spoke element. If $k>j+1$, then $T$ intersects the triad $\left\{e_{j-1}, e_{j}, e_{j+1}\right\}$ in a single element. Therefore $k=j+1$, implying that $e_{k}$ is a rim element. Since this contradicts our earlier conclusion, we see that $j=i+1$. Now $k=i+2$, by orthogonality between $T$ and $\left\{e_{i+1}, e_{i+2}, e_{i+3}\right\}$, and the fact that $e_{k}$ is a spoke element.

Proposition 5.7. Let $M$ be a 3-connected matroid such that $|E(M)| \geqslant 4$ and $M$ is neither a wheel nor a whirl. Let $\left(e_{1}, \ldots, e_{n}\right)$ be a fan of $M$. If $X \subseteq\left\{e_{1}, \ldots, e_{n}\right\}$ is a 3 -separating set of $M$ such that $|X| \geqslant 3$, then there are integers $1 \leqslant x<y \leqslant n$ such that $X=\left\{e_{x}, e_{x+1}, \ldots, e_{y}\right\}$.

Proof. Assume that the result fails for $X$, and that $X$ is as large as possible with respect to this assumption. If $|E(M)|=4$, then $M$ is $U_{2,4}$, contradicting the fact that $M$ is not a whirl. If $|E(M)|=5$, then $M$ is $U_{2,5}$ or $U_{3,5}$. In the first case $M$ has no triads, and in the second, $M$ has no triangles. In either case, $n=3$, and $X=\left\{e_{1}, e_{2}, e_{3}\right\}$. Therefore we may 
as well assume that $|E(M)| \geqslant 6$. Let $i$ be the least index such that $\left|X \cap\left\{e_{1}, \ldots, e_{i}\right\}\right|=3$. Then $X \cup\left\{e_{1}, \ldots, e_{i}\right\}$ is contained in $\left\{e_{1}, \ldots, e_{n}\right\}$, so the complement of $X \cup\left\{e_{1}, \ldots, e_{i}\right\}$ contains at least two elements, or else $M$ is a wheel or a whirl by Proposition 5.4. As $M$ is 3-connected, it follows that $X \cup\left\{e_{1}, \ldots, e_{i}\right\}$ is not 2-separating. As $\lambda\left(\left\{e_{1}, \ldots, e_{i}\right\}\right) \leqslant$ $2, \lambda(X) \leqslant 2$, and $\lambda\left(X \cup\left\{e_{1}, \ldots, e_{i}\right\}\right) \geqslant 2$, it follows that $X^{\prime}=X \cap\left\{e_{1}, \ldots, e_{i}\right\}$ is a 3 -separating set with cardinality three, by the submodularity of $\lambda$ ([9, Lemma 8.2.9]). Since $|E(M)| \geqslant 6$, it follows that $\left(X^{\prime}, E(M)-X^{\prime}\right)$ is a 3 -separation of $M$. Thus $X^{\prime}$ is a triad or a triangle $\left(\left[9\right.\right.$, Corollary 8.2.2]). By duality, we will assume that $X^{\prime}$ is a triangle of $M$. Proposition 5.6 implies that $X^{\prime}=\left\{e_{i-2}, e_{i-1}, e_{i}\right\}$.

There must be an index $p>i$ such that $e_{p} \notin X$, or else $X$ is not a counterexample. Let $p$ be the least such index. Note that $e_{p-2}$ and $e_{p-1}$ are in $X$. Therefore $e_{p}$ is in either $\mathrm{cl}_{M}(X)$ or $\mathrm{cl}_{M}^{*}(X)$, so $X \cup e_{p}$ is 3 -separating. Because $X \cup e_{p}$ is strictly larger than $X$, it follows that $X \cup e_{p}$ is not a counterexample, so $X \cup e_{p}=\left\{e_{x}, e_{x+1}, \ldots, e_{y}\right\}$ for some indices $x$ and $y$. Clearly $p<y$, or else $X=\left\{e_{x}, e_{x+1}, \ldots, e_{y-1}\right\}$. Thus $e_{p+1}$ is in $X$. Now $\left\{e_{p-2}, e_{p-1}, e_{p}\right\}$ and $\left\{e_{p-1}, e_{p}, e_{p+1}\right\}$ show that $e_{p}$ is in the closure and the coclosure of $X$. Therefore $\lambda\left(X \cup e_{p}\right) \leqslant 1$, so the complement of $X \cup e_{p}$ contains at most one element. Hence $M$ is a wheel or a whirl, and this contradiction completes the proof.

Lemma 5.8. Let $M$ be a 3-connected matroid such that $|E(M)| \geqslant 4$ and $M$ is not a wheel or a whirl. Let $\left(f_{1}, \ldots, f_{m}\right)$ and $\left(g_{1}, \ldots, g_{n}\right)$ be fans of $M$. Assume that $\mid\left\{f_{1}, \ldots, f_{m}\right\} \cap$ $\left\{g_{1}, \ldots, g_{n}\right\} \mid \geqslant 3$. Then there are integers $1 \leqslant x<y \leqslant m$ and $1 \leqslant x^{\prime}<y^{\prime} \leqslant n$ such that

$$
\left\{f_{1}, \ldots, f_{m}\right\} \cap\left\{g_{1}, \ldots, g_{n}\right\}=\left\{f_{x}, f_{x+1}, \ldots, f_{y}\right\}=\left\{g_{x^{\prime}}, g_{x^{\prime}+1}, \ldots, g_{y^{\prime}}\right\} .
$$

Proof. The proof is by induction on $m+n$. The hypotheses imply that $m+n \geqslant 6$. If $m+n=6$, then obviously $m=n=3$ and

$$
\left\{f_{1}, f_{2}, f_{3}\right\} \cap\left\{g_{1}, g_{2}, g_{3}\right\}=\left\{f_{1}, f_{2}, f_{3}\right\}=\left\{g_{1}, g_{2}, g_{3}\right\}
$$

so there is nothing left to prove. Let us assume that $m+n>6$, and that the result does not hold for $\left(f_{1}, \ldots, f_{m}\right)$ and $\left(g_{1}, \ldots, g_{n}\right)$. We will make the inductive assumption that the result is true for any pair of fans with combined length less than $m+n$.

Let $F=\left\{f_{1}, \ldots, f_{m}\right\}$, and let $G=\left\{g_{1}, \ldots, g_{n}\right\}$.

5.8.1. $m, n>3$.

Proof. By symmetry, it suffices to prove that $m>3$. If $m=3$, then $F \cap G=\left\{f_{1}, f_{2}, f_{3}\right\}$. Moreover, $F$ is a triangle or a triad contained in $G$. Proposition 5.6 or its dual tells us that $F=F \cap G$ consists of three consecutive elements from $\left(g_{1}, \ldots, g_{n}\right)$. Therefore the result holds, contrary to our choice of $\left(f_{1}, \ldots, f_{m}\right)$ and $\left(g_{1}, \ldots, g_{n}\right)$.

5.8.2. $f_{1}, f_{m} \in G$ and $g_{1}, g_{n} \in F$.

Proof. Suppose that $f_{1} \notin G$. Then $\left(f_{2}, \ldots, f_{m}\right)$ and $\left(g_{1}, \ldots, g_{n}\right)$ are fans by 5.8.1. Since $\left|\left\{f_{2}, \ldots, f_{m}\right\} \cap\left\{g_{1}, \ldots, g_{n}\right\}\right| \geqslant 3$, the inductive assumption implies that there are integers 
$1 \leqslant x<y \leqslant m$ and $1 \leqslant x^{\prime}<y^{\prime} \leqslant n$ such that

$$
\begin{aligned}
\left\{f_{1}, \ldots, f_{m}\right\} \cap\left\{g_{1}, \ldots, g_{n}\right\}=\left\{f_{2}, \ldots, f_{m}\right\} & \cap\left\{g_{1}, \ldots, g_{n}\right\} \\
& =\left\{f_{x}, f_{x+1}, \ldots, f_{y}\right\}=\left\{g_{x^{\prime}}, g_{x^{\prime}+1}, \ldots, g_{y^{\prime}}\right\},
\end{aligned}
$$

and the result holds, contrary to assumption. Exactly the same argument shows that $f_{m} \in G$ and $g_{1}, g_{n} \in F$.

Let the ground set of $M$ be $E$.

5.8.3. $|E-(F \cup G)| \leqslant 1$.

Proof. Assume that $|E-(F \cup G)| \geqslant 2$. As $|F \cup G| \geqslant 2$, and $M$ is 3-connected, it follows that $\lambda(F \cup G) \geqslant 2$. Since $\lambda(F), \lambda(G) \leqslant 2$, the submodularity of $\lambda$ shows that $\lambda(F \cap G) \leqslant 2$. Then we can set $X=F \cap G$ and apply Proposition 5.7 to see that

$$
F \cap G=\left\{f_{x}, f_{x+1}, \ldots, f_{y}\right\}=\left\{g_{x^{\prime}}, g_{x^{\prime}+1}, \ldots, g_{y^{\prime}}\right\}
$$

for integers $1 \leqslant x<y \leqslant m$ and $1 \leqslant x^{\prime}<y^{\prime} \leqslant n$. This contradiction completes the proof of the claim.

5.8.4. $|F-G|,|G-F| \geqslant 2$.

Proof. We have complete symmetry between $F$ and $G$, so it suffices to prove that $|F-G| \geqslant$ 2. Assume that $|F-G| \leqslant 1$. If $|F-G|=0$, then $|E-G| \leqslant 1$ by 5.8 .3 , so Proposition 5.4 implies that $M$ is a wheel or a whirl. This contradiction means that $|F-G|=1$. Let $f_{i}$ be the unique element in $F-G$. By 5.8.2, we deduce that $1<i<m$. Either $\left\{f_{i-1}, f_{i}, f_{i+1}\right\}$ and $\left\{f_{i}, f_{i+1}, f_{i+2}\right\}$ are a triangle and a triad that are contained in $G \cup f_{i}$, or we can make the same statement about $\left\{f_{i-1}, f_{i}, f_{i+1}\right\}$ and $\left\{f_{i-2}, f_{i-1}, f_{i}\right\}$. This means that we can apply Proposition 5.5, and deduce that $M$ is a wheel or a whirl. This contradiction completes the proof of 5.8.4.

5.8.5. The elements of $F-G$ form a consecutive subsequence of $\left(f_{1}, \ldots, f_{m}\right)$. The elements of $G-F$ form a consecutive subsequence of $\left(g_{1}, \ldots, g_{n}\right)$.

Proof. It suffices to prove that the elements in $F-G$ form a consecutive subsequence of $\left(f_{1}, \ldots, f_{m}\right)$. Certainly $F \cup(E-G)$ contains at least two elements. Its complement, $G-F$, contains at least two elements by 5.8.4. Therefore $\lambda(F \cup(E-G)) \geqslant 2$. As $\lambda(F), \lambda(E-G) \leqslant 2$, it follows that $\lambda(F \cap(E-G))=\lambda(F-G) \leqslant 2$.

If $|F-G| \geqslant 3$, then we can let $X=F-G$, and apply Proposition 5.7 to the fan $\left(f_{1}, \ldots, f_{m}\right)$. Therefore we assume $|F-G|=2$. Let $i, j \in\{1, \ldots, m\}$ be chosen so that $i<j$, and $\left\{f_{i}, f_{j}\right\}=F-G$. By 5.8.2, $1<i<j<m$. If $j=i+1$, there is nothing left to prove, so $j>i+1$. Assume that $j>i+2$. Then $\left\{f_{i-1}, f_{i}, f_{i+1}\right\}$ and $\left\{f_{i}, f_{i+1}, f_{i+2}\right\}$ are a triangle or triad contained in $G \cup f_{i}$. Proposition 5.5 implies that $M$ is a wheel or a whirl. Hence $j=i+2$.

By duality, we can assume that $f_{i}$ is a rim element of $\left(f_{1}, \ldots, f_{m}\right)$. Since $\left\{f_{i-1}, f_{i}, f_{i+1}\right\}$ is a triangle that contains $f_{i}$ and is otherwise contained in $G$, we can apply Proposition 5.3. 
From 5.8.4 and the fact that $|F \cap G| \geqslant 3$, we see that $m, n \geqslant 5$. Thus $\left\{f_{i-1}, f_{i+1}\right\}$ is $\left\{g_{1}, g_{2}\right\},\left\{g_{n-1}, g_{n}\right\},\left\{g_{1}, g_{n}\right\}$, or $\left\{g_{2}, g_{4}\right\}$. But $\left\{f_{i+1}, f_{i+2}, f_{i+3}\right\}$ is a triangle, and we can apply the same arguments to show that $\left\{f_{i+1}, f_{i+3}\right\}$ is also one of the same four sets. We deduce that $\left\{\left\{f_{i-1}, f_{i+1}\right\},\left\{f_{i+1}, f_{i+3}\right\}\right\}$ is one of $\left\{\left\{g_{1}, g_{2}\right\},\left\{g_{1}, g_{n}\right\}\right\},\left\{\left\{g_{1}, g_{n}\right\},\left\{g_{n-1}, g_{n}\right\}\right\}$, $\left\{\left\{g_{1}, g_{2}\right\},\left\{g_{2}, g_{4}\right\}\right\}$, or $\left\{\left\{g_{2}, g_{4}\right\},\left\{g_{4}, g_{5}\right\}\right\}$. Proposition 5.3 implies that, in the first case, $g_{1}$ is both a spoke element and a rim element. In the second case it implies $g_{n}$ is a spoke element and a rim element. In the third case, $g_{2}$ is both a rim element and a spoke element, and in the fourth, $g_{4}$ is a rim element and a spoke element. Thus we have a contradiction in any case.

By 5.8.2, 5.8.4, and 5.8.5, there are indices $1 \leqslant i<i+3 \leqslant j \leqslant m$ such that $F \cap G=\left\{f_{1}, \ldots, f_{i}\right\} \cup\left\{f_{j} \ldots, f_{m}\right\}$. Note that $\left\{f_{i}, f_{i+1}, f_{i+2}\right\}$ is a triangle or a triad that contains exactly one element of $G$. If $f_{i}=g_{k}$, then $k=1$ or $k=n$, for otherwise, since $n \geqslant 4$, it follows that $g_{k}$ is contained in a triangle and a triad that are both contained in $G$. This leads to a contradiction to orthogonality. By applying the same arguments to $\left\{f_{j-2}, f_{j-1}, f_{j}\right\}$, we see that $f_{j}$ is equal to either $g_{1}$ or $g_{n}$. By reversing $\left(g_{1}, \ldots, g_{n}\right)$ as necessary, we can assume that $f_{i}=g_{n}$ and that $f_{j}=g_{1}$.

Assume that $|F \cap G| \geqslant 4$. Either $1<i$ or $j<m$. Let us first assume $1<i$. The fans $\left(f_{1}, \ldots, f_{m}\right)$ and $\left(g_{1}, \ldots, g_{n-1}\right)$ intersect in $\left\{f_{1}, \ldots, f_{i-1}\right\} \cup\left\{f_{j}, \ldots, f_{m}\right\}$, and this set contains at least three elements. The inductive assumption implies that $\left(f_{1}, \ldots, f_{m}\right)$ and $\left(g_{1}, \ldots, g_{n-1}\right)$ should meet in consecutive subsequences of $\left(f_{1}, \ldots, f_{m}\right)$ and $\left(g_{1}, \ldots, g_{n-1}\right)$, but $G-g_{n}$ contains $f_{i-1}$ and $f_{j}$, and no element between them. Similarly, if $j<m$, then $\left(f_{1}, \ldots, f_{m}\right)$ and $\left(g_{2}, \ldots, g_{n}\right)$ intersect in $\left\{f_{1}, \ldots, f_{i}\right\} \cup\left\{f_{j+1}, \ldots, f_{m}\right\}$, and this set is not consecutive in $\left(f_{1}, \ldots, f_{m}\right)$. From this contradiction to the inductive hypothesis, we deduce that $|F \cap G|=3$. Either $i=1$ and $j=m-1$, or $i=2$ and $j=m$. We can reverse $\left(f_{1}, \ldots, f_{m}\right)$ and $\left(g_{1}, \ldots, g_{n}\right)$, and assume that the former case holds. Thus $f_{1}=g_{n}$ and $f_{m-1}=g_{1}$. Since $\left\{f_{m-2}, f_{m-1}, f_{m}\right\}$ is a triangle or a triad contained in $G \cup f_{m-2}$ that contains $g_{1}$ but not $g_{n}$, Proposition 5.3 implies that $f_{m}=g_{2}$. Hence $F \cap G=\left\{f_{1}, f_{m-1}, f_{m}\right\}=\left\{g_{n}, g_{1}, g_{2}\right\}$.

By replacing $M$ with $M^{*}$, we can assume that $f_{m}=g_{2}$ is a spoke element of $\left(f_{1}, \ldots, f_{m}\right)$, so that $\left\{f_{m-2}, f_{m-1}, f_{m}\right\}$ is a triangle. Note that $\left\{g_{1}, g_{2}, g_{3}\right\}$ is not a triangle, or else it intersects the triad $\left\{f_{m-3}, f_{m-2}, f_{m-1}\right\}$ in the single element $f_{m-1}=g_{1}$. Therefore $g_{1}$ is a rim element of $\left(g_{1}, \ldots, g_{n}\right)$. It follows that

$$
\left(f_{1}, \ldots, f_{m-2}, f_{m-1}, f_{m}, g_{3}, \ldots, g_{n-1}\right)=\left(f_{1}, \ldots, f_{m-2}, g_{1}, g_{2}, g_{3}, \ldots, g_{n-1}\right)
$$

is a fan of $M$. By 5.8.3, there is at most one element of $E$ not contained in this fan, so $M$ is a wheel or a whirl. This contradiction completes the proof of Lemma 5.8.

\section{A finite case-check theorem}

From this point onwards, $\mathcal{M}$ will be a class of matroids closed under isomorphism and minors, and $N \in \mathcal{M}$ will be a fixed 3-connected matroid such that $|E(N)| \geqslant 4$, and $N$ is neither a wheel nor a whirl. We let $\mathcal{F}_{N}$ be a family of disjoint fans in $N$. Assume that 
whenever $M^{\prime} \in \mathcal{M}$ has $N$ as a minor, $M^{\prime}$ is 3-connected up to series and parallel sets. Note that we can replace $\mathcal{M}$ with the dual class $\left\{M^{*} \mid M \in \mathcal{M}\right\}$ and replace $N$ with $N^{*}$ and the same hypotheses will hold.

In the subsequent results we state some consequences of these definitions. First of all, the only 3-connected minors of wheels and whirls are wheels and whirls. This has the following implication.

Proposition 6.1. No matroid having $N$ as a minor is a wheel or a whirl.

We are going to make continuous use of the next two results.

Proposition 6.2. Let $M^{\prime}$ be a matroid in $\mathcal{M}$ that has $N$ as a minor. Every triangle in $M^{\prime}$ is coindependent, and every triad is independent.

Proof. By duality, it suffices to assume that $T$ is a codependent triangle in $M^{\prime}$. Then $r_{M^{\prime}}(T)=2$, and $r_{M^{\prime}}^{*}(T)<3$, so $\lambda_{M^{\prime}}(T) \leqslant 1$. Clearly $T$ is not contained in a parallel class. If $T$ is contained in a series class, then $\lambda_{M^{\prime}}(T)=0$, so $E\left(M^{\prime}\right)=T$ since $M^{\prime}$ is connected. This contradicts the fact that $N$ is a minor of $M^{\prime}$. As $M^{\prime}$ is 3-connected up to series and parallel sets, the complement of $T$ is contained in a series or parallel class. This implies that a 3-connected minor of $M^{\prime}$ with at least four elements has precisely four elements. Hence $|E(N)|=4$, and $N$ is isomorphic to $U_{2,4}$, which contradicts the assumption that $N$ is not a whirl.

The next result follows from Proposition 6.2 by orthogonality.

Corollary 6.3. Let $M^{\prime}$ be a matroid in $\mathcal{M}$ that has $N$ as a minor. If $X$ is a $U_{2,4}$-restriction of $M^{\prime}$, and $T^{*}$ is a triad of $M^{\prime}$, then $X \cap T^{*}=\emptyset$.

Proposition 6.4. Let $M^{\prime}$ be a 3-connected matroid in $\mathcal{M}$. Assume that $\left(e_{1}, \ldots, e_{n}\right)$ is a fan of $M^{\prime}$ such that $n \geqslant 4$, and $N$ is a minor of $M^{\prime} / e_{i} \backslash e_{i+1}$ for some $i \in\{1, \ldots, n-1\}$. Then $M^{\prime} / e_{i} \backslash e_{i+1}$ is 3-connected.

Proof. If $M^{\prime} / e_{i} \backslash e_{i+1}$ is not 3-connected, then, as it is 3-connected up to series and parallel sets, there is a triangle that contains $e_{i}$ but not $e_{i+1}$, or a triad that contains $e_{i+1}$ but not $e_{i}$. We assume $T^{*}$ is a triad satisfying $T^{*} \cap\left\{e_{i}, e_{i+1}\right\}=\left\{e_{i+1}\right\}$.

First assume that $e_{i+1}$ is a rim element. If $i>2$, then $\left\{e_{i-2}, e_{i-1}, e_{i}\right\}$ is a codependent triangle in $M^{\prime} \backslash e_{i+1}$, contradicting Proposition 6.2. Similarly, if $i<n-2$, then $\left\{e_{i+1}, e_{i+2}, e_{i+3}\right\}$ is a dependent triad in $M^{\prime} / e_{i}$. Therefore $n-2 \leqslant i \leqslant 2$, implying that $n=4$ and $i=2$. Since $e_{i} \notin T^{*}$, orthogonality with $\left\{e_{i}, e_{i+1}, e_{i+2}\right\}$ implies $T^{*}$ contains the parallel pair $\left\{e_{i+1}, e_{i+2}\right\}$ in $M^{\prime} / e_{i}$, and we have another contradiction to Proposition 6.2.

Thus $e_{i+1}$ is a spoke element. If $\left\{e_{i}, e_{i+1}\right\}$ is contained in a triangle, then $T^{*}$ contains the third element of this triangle, and $T^{*}$ is a triad of $M^{\prime} / e_{i}$ that contains a parallel pair, contradicting Proposition 6.2. Therefore there is no triangle containing $\left\{e_{i}, e_{i+1}\right\}$, so $i=1$. In this case $T^{*}$ contains two elements of the triangle $\left\{e_{i+1}, e_{i+2}, e_{i+3}\right\}$. In a matroid without series pairs, two triads that intersect in two elements form a $U_{2,4}$-corestriction. Therefore, if $T^{*}$ contains $e_{i+2}$, then $T^{*} \cup e_{i}$ is a $U_{2,4}$-corestriction of $M^{\prime}$ that intersects the 
triangle $\left\{e_{i+1}, e_{i+2}, e_{i+3}\right\}$, contradicting Corollary 6.3. Therefore $T^{*} \cap\left\{e_{i+1}, e_{i+2}, e_{i+3}\right\}=$ $\left\{e_{i+1}, e_{i+3}\right\}$. Let $M^{\prime \prime}$ be produced from $M^{\prime}$ by swapping labels on $e_{i}$ and $e_{i+2}$. As $\left\{e_{i}, e_{i+2}\right\}$ is a series pair in $M^{\prime} \backslash e_{i+1}$, it follows that $M^{\prime \prime} \backslash e_{i+1}=M^{\prime} \backslash e_{i+1}$, so $M^{\prime \prime} \backslash e_{i+1} / e_{i}$ has $N$ as a minor. However, $M^{\prime \prime} / e_{i}$ contains the parallel pair $\left\{e_{i+1}, e_{i+3}\right\}$, which is contained in the triad $T^{*}$. This contradicts Proposition 6.2.

To complete the proof, we must assume that there is a triangle containing $e_{i}$ but not $e_{i+1}$. In this case we replace $M^{\prime}, N$, and $\mathcal{M}$ by their duals, and we reverse the fan $\left(e_{1}, \ldots, e_{n}\right)$, relabeling $e_{n-i}$ as $e_{i}$. After the relabeling, there is a triad that contains $e_{i+1}$ but not $e_{i}$, so we make the same argument as before.

Proposition 6.5. Let $M^{\prime}$ be a matroid in $\mathcal{M}$ with $N$ as a minor. Assume $M^{\prime}$ has a covering family, and that $F=\left(e_{1}, \ldots, e_{n}\right)$ is a fan in that family, where $\left\{e_{i}, e_{i+1}\right\} \subseteq$ $E\left(M^{\prime}\right)-E(N)$ for some rim element $e_{i}$. Then $N$ is a minor of $M^{\prime} / e_{i} \backslash e_{i+1}$.

Proof. Note that $n \geqslant 5$, since $F$ contains a fan in $\mathcal{F}_{N}$ as well as $\left\{e_{i}, e_{i+1}\right\}$. If $N$ is a minor of $M^{\prime} \backslash e_{i}$, then $n=5$ and $i=3$, for otherwise $\left\{e_{i-3}, e_{i-2}, e_{i-1}\right\}$ or $\left\{e_{i+1}, e_{i+2}, e_{i+3}\right\}$ is a codependent triangle in $M^{\prime} \backslash e_{i}$. But now $F-\left\{e_{i}, e_{i+1}\right\} \subseteq E(N)$, so $N$ contains the series pair $\left\{e_{i-2}, e_{i-1}\right\}$. Therefore $N$ is a minor of $M^{\prime} / e_{i}$. If $i>1$, then $N$ is a minor of $M^{\prime} / e_{i} \backslash e_{i+1}$, as $\left\{e_{i-1}, e_{i+1}\right\}$ is a parallel pair in $M^{\prime} / e_{i}$. If $i=1$, then $\left\{e_{3}, e_{4}, e_{5}\right\}$ is a dependent triad in $M^{\prime} / e_{i} / e_{i+1}$, so $N^{\prime}$ is a minor of $M^{\prime} / e_{i} \backslash e_{i+1}$ in either case.

Proposition 6.6. Let $M^{\prime}$ be a 3-connected matroid in $\mathcal{M}$ with $N$ as a minor. Assume that $M^{\prime} / x \backslash y$ is 3-connected and has $N$ as a minor. Let $F$ be a fan of $M^{\prime}$ such that a fan in $\mathcal{F}_{N}$ is consistent with $F$. If either $x$ or $y$ is an internal element of $F$, then they are consecutive in $F$, and $x$ is a rim element.

Proof. Let $F=\left(e_{1}, \ldots, e_{m}\right)$, and assume $x$ or $y$ is an internal element in $F$. In the latter case, we swap $M^{\prime}, N$, and $\mathcal{M}$ for their duals, and swap labels on $x$ and $y$. Therefore we lose no generality in assuming $x$ is an internal element, $e_{i}$, of $F$. Assume $x$ is a spoke element. If $n>5$, then $\left\{e_{i-3}, e_{i-2}, e_{i-1}\right\}$ or $\left\{e_{i+1}, e_{i+2}, e_{i+3}\right\}$ is a dependent triad in $M^{\prime} / x$, which is a contradiction. Therefore $n \leqslant 5$. If $n=4$, then $F-x \subseteq E(N)$, as $F$ contains a fan in $\mathcal{F}_{N}$, as well as $x$. But $F-x$ also contains a parallel pair in $M^{\prime} / x$. This leads to the contradiction that $N$ contains a parallel pair. Therefore $n=5$, and $x=e_{3}$, for otherwise $M^{\prime} / x$ contains a dependent triad. Now either $\left\{e_{1}, e_{2}\right\}$ or $\left\{e_{4}, e_{5}\right\}$ is contained in $E(N)$, and as both these sets are parallel pairs in $M^{\prime} / x$, this is a contradiction.

Hence $x$ is a rim element in $F$. Because $\left\{e_{i-1}, e_{i+1}\right\}$ is a parallel pair in $M^{\prime} / x$, and $M^{\prime} / x \backslash y$ is 3-connected, it follows that $y \in\left\{e_{i-1}, e_{i+1}\right\}$, and we are done.

Proposition 6.7. Let $M^{\prime}$ be a 3-connected member of $\mathcal{M}$, and assume that $\left(e_{1}, \ldots, e_{n}\right)$ is a fan of $M^{\prime}$ containing $x, y$, and $z$, where $\{x, y, z\}$ is a triangle of $M^{\prime}$, and $N$ is a minor of $M^{\prime} / x \backslash y$. If $n \geqslant 5$, then $x$ and $y$ are consecutive elements in $\left(e_{1}, \ldots, e_{n}\right)$.

Proof. By Proposition 5.6, there is some $i \in\{1, \ldots, n-2\}$ such that $\{x, y, z\}=$ $\left\{e_{i}, e_{i+1}, e_{i+2}\right\}$, where $e_{i}$ is a spoke element. By reversing $\left(e_{1}, \ldots, e_{n}\right)$ as necessary, we can assume that $(x, y)=\left(e_{i}, e_{i+2}\right)$, for otherwise there is nothing left to prove. If $i+2<n$, 
then $\left\{e_{i+1}, e_{i+2}, e_{i+3}\right\}$ is a dependent triad of $M^{\prime} / e_{i}=M^{\prime} / x$, since $\left\{e_{i+1}, e_{i+2}\right\}$ is a parallel pair. This is impossible, as $M^{\prime} / x$ has $N$ as a minor. Therefore $n=i+2$.

Let $M^{\prime \prime}$ be the matroid obtained from $M^{\prime}$ by swapping the labels on $e_{i+1}=z$ and $e_{i+2}=y$. Since $\{y, z\}$ is a parallel pair in $M^{\prime} / x$, it follows that $M^{\prime \prime} / x \backslash y=M^{\prime} / x \backslash y$. Therefore $N$ is a minor of $M^{\prime \prime} \backslash y$. But $\left\{e_{i-2}, e_{i-1}, e_{i}\right\}$ is a triangle of $M^{\prime \prime} \backslash y$ that contains a series pair. This contradiction completes the proof.

Proposition 6.8. Let $M^{\prime} \in \mathcal{M}$ be a 3-connected matroid with $N$ as a minor. If $e \in$ $E\left(M^{\prime}\right)-E(N)$, then for some $\left(M_{1}, N_{1}\right)$ in $\left\{\left(M^{\prime}, N\right),\left(\left(M^{\prime}\right)^{*}, N^{*}\right)\right\}$ one of the following statements holds:

(i) $M_{1} / e$ is 3-connected and has $N_{1}$ as a minor,

(ii) there is a triangle $\{x, e, f\}$ of $M_{1}$ such that $N_{1}$ is a minor of $M_{1} / e \backslash f$ and $M_{1} \backslash f$ is 3-connected,

(iii) there is a fan $(x, e, f, y)$ of $M_{1}$, where $\{x, e, f\}$ is a triangle, and $M_{1} / e \backslash f$ is 3-connected with $N_{1}$ as a minor.

Proof. By duality, we can assume $N_{1}$ is a minor of $M_{1} / e$. We assume $M_{1} / e$ is not 3-connected. Since $M_{1}$ is 3-connected, and $M_{1} / e$ is 3-connected up to series and parallel sets, $M_{1}$ has a triangle containing $e$. Let this triangle be $\{x, e, f\}$, where $N_{1}$ is a minor of $M_{1} / e \backslash f$. We assume $M_{1} \backslash f$ is not 3-connected, so $f$ is in a triad of $M_{1}$. This triad contains $x$ or $e$ by orthogonality, and does not contain both by Proposition 6.2. Let $y$ be the third element in the triad. If $\{x, f, y\}$ is a triad, then it is a dependent triad in $M_{1} / e$, which is impossible, so $\{e, f, y\}$ is a triad, and statement (iii) holds by Proposition 6.4.

Proposition 6.9. Let $N^{\prime}$ be a matroid in $\mathcal{M}$ such that $\left(e_{1}, e_{2}, e_{3}, e_{4}\right)$ is a fan of $N^{\prime}$ with $e_{2}$ as a rim element, and $N^{\prime} / e_{2} \backslash e_{3}=N$. Then $N^{\prime}$ is 3-connected.

Proof. Because $N^{\prime}$ has $N$ as a minor, it is 3-connected up to series and parallel sets, so it is connected. In particular, it has no loops or coloops. If $e_{2}$ is in a parallel pair, then $N^{\prime} / e_{2}$ has $N$ as a minor and a loop, which is impossible. If $e_{2}$ is in a series pair, then orthogonality requires that the pair is contained in $\left\{e_{1}, e_{2}, e_{3}\right\}$, so this set is a codependent triangle in $N^{\prime}$, a contradiction to Proposition 6.2. By a dual argument, $e_{3}$ is not contained in any series or parallel pair in $N$. As $N=N^{\prime} / e_{2} \backslash e_{3}$ is simple and cosimple, it follows that $N^{\prime}$ is simple and cosimple.

Assume $N^{\prime}$ is not 3-connected. As it is connected, we can let $(X, Y)$ be a 2-separation. Since $N^{\prime}$ is simple and cosimple, it follows that $|X|,|Y| \geqslant 3$. If $X \cap\left\{e_{2}, e_{3}\right\}$ and $Y \cap\left\{e_{2}, e_{3}\right\}$ are both non-empty, then $\left(X-\left\{e_{2}, e_{3}\right\}, Y-\left\{e_{2}, e_{3}\right\}\right)$ is a 2 -separation in $N$, a contradiction. Therefore we can assume $e_{2}, e_{3} \in X$. If $|X|>3$, then $\left(X-\left\{e_{2}, e_{3}\right\}, Y\right)$ is a 2-separation in $N$. Therefore $|X| \leqslant 3$. If $|E(N)|=4$, then $N$ is isomorphic to $U_{2,4}$, contradicting the hypothesis that it is not a whirl. Hence $\left|E\left(N^{\prime}\right)\right|=|E(N)|+2 \geqslant 7$, so $|Y| \geqslant 4$. Now $\left(X \cup\left\{e_{1}, e_{4}\right\}, Y-\left\{e_{1}, e_{4}\right\}\right)$ is a 2-separation in $N^{\prime}$ that induces a 2-separation in $N$, so we have a contradiction that completes the proof. 
Now we can begin the proof of our main theorem.

Theorem 6.10. Let $\mathcal{M}$ be a set of matroids that is closed under minors and isomorphism. Let $N \in \mathcal{M}$ be a 3-connected matroid such that $|E(N)| \geqslant 4$ and $N$ is neither a wheel nor a whirl. Assume that any member of $\mathcal{M}$ with $N$ as a minor is 3-connected up to series and parallel sets. Let $\mathcal{F}_{N}$ be a family of pairwise disjoint fans of $N$. If there is a 3-connected matroid in $\mathcal{M}$ with $N$ as a minor that is not a fan-extension of $N$ relative to $\mathcal{F}_{N}$, then there exists such a matroid, $M$, satisfying $|E(M)|-|E(N)| \leqslant 2$.

For the remainder of the paper, we let $\mathcal{M}, N$, and $\mathcal{F}_{N}$ be as in the statement of Theorem 6.10. We let $M \in \mathcal{M}$ be a 3-connected matroid with $N$ as a minor such that $M$ is not a fan-extension of $N$, and, subject to these constraints, $|E(M)|$ is as small as possible. We assume that $|E(M)|-|E(N)|>2$, and ultimately derive a contradiction from this, thereby proving Theorem 6.10 .

Lemma 6.11. Let $M_{0}$ be isomorphic to $M$, and assume that $M_{0}$ has $N$ as a minor, but is not a fan-extension of $N$. Then $M_{0}$ does not have a covering family (relative to $N$ and $\left.\mathcal{F}_{N}\right)$.

Proof. We assume for a contradiction that $M_{0}$ has a covering family. Note $M_{0} \neq N$, as $M_{0}$ is not a fan-extension of $N$. The fans in any covering family of $M_{0}$ contain the elements of $E\left(M_{0}\right)-E(N)$.

6.11.1. In any fan belonging to a covering family of $M_{0}$, there are no two consecutive elements in $E\left(M_{0}\right)-E(N)$.

Proof. Assume there is a covering family, $\mathcal{F}$, of $M_{0}$, and a fan, $F=\left(e_{1}, \ldots, e_{n}\right)$, in $\mathcal{F}$, such that $e_{i}$ and $e_{i+1}$ are not in $E(N)$ for some $i \in\{1, \ldots, n-1\}$. If $e_{i}$ is a spoke element, then we can replace $M, M_{0}, N$, and $\mathcal{M}$ by their duals, and then apply the forthcoming arguments. Thus we assume that $e_{i}$ is a rim element. Then $N$ is a minor of $M_{0} / e_{i} \backslash e_{i+1}$ by Proposition 6.5. Propositions 5.2 and 6.4 show that $M_{0} / e_{i} \backslash e_{i+1}$ is a 3-connected matroid containing the fan $F-\left\{e_{i}, e_{i+1}\right\}$. The minimality of $M$ implies $M_{0} / e_{i} \backslash e_{i+1}$ is a fan-extension of $N$. It is easy to see that $(\mathcal{F}-\{F\}) \cup\left\{F-\left\{e_{i}, e_{i+1}\right\}\right\}$ is a covering family in $M_{0} / e_{i} \backslash e_{i+1}$, and $M_{0}$ is obtained from $M_{0} / e_{i} \backslash e_{i+1}$ by a fan-lengthening move on $F-\left\{e_{i}, e_{i+1}\right\}$. Therefore $M_{0}$ is a fan-extension of $N$, a contradiction.

6.11.2. If $F$ is a fan in a covering family of $M_{0}$, then all internal elements of $F$ belong to $E(N)$.

Proof. Assume $F=\left(e_{1}, \ldots, e_{n}\right)$ is a fan in a covering family, and $e_{i}$ is an internal element in $E\left(M_{0}\right)-E(N)$, so $n \geqslant 4$. By replacing $M, M_{0}, N$, and $\mathcal{M}$ with their duals if necessary, we can assume $e_{i}$ is a rim element. If $N$ is a minor of $M_{0} / e_{i}$, we contradict 6.11.1, as $\left\{e_{i-1}, e_{i+1}\right\}$ is a parallel pair in $M_{0} / e_{i}$, so $N$ is a minor of $M_{0} / e_{i} \backslash e_{i-1}$ or $M_{0} / e_{i} \backslash e_{i+1}$. Therefore $N$ is a minor of $M_{0} \backslash e_{i}$, so $n \leqslant 5$, and if $n=5$, then $i=3$, for otherwise $\left\{e_{i-3}, e_{i-2}, e_{i-1}\right\}$ or $\left\{e_{i+1}, e_{i+2}, e_{i+3}\right\}$ is a codependent triangle in $M_{0} \backslash e_{i}$. By reversing $F$ as required, we will assume that $i<n-1$. Now $\left\{e_{i+1}, e_{i+2}\right\}$ is a series pair in $M_{0} \backslash e_{i}$, so 
6.11.1 means that $N$ is a minor of $M_{1} \backslash e_{i} / e_{i+2}$. Thus $n=5$, since $F$ contains $\left\{e_{i}, e_{i+2}\right\}$ and at least three elements of $E(N)$. In this case, $\left\{e_{i-2}, e_{i-1}, e_{i+1}\right\} \subseteq E(N)$, which leads to a contradiction as $\left\{e_{i-2}, e_{i-1}\right\}$ is is a series pair in $M_{0} \backslash e_{i}$.

Fix the covering family $\mathcal{F}$, and let $F=\left(e_{1}, \ldots, e_{n}\right)$ be a fan in $\mathcal{F}$ such that $e_{1} \notin E(N)$. This implies $n \geqslant 4$. By duality, we assume $e_{1}$ is a rim element, and this easily implies that $N$ is a minor of $M_{0} / e_{1}$. If $M_{0} / e_{1}$ is 3-connected, then $M_{0}$ is obtained from $M_{0} / e_{1}$ by a fan-lengthening move on $F-e_{1}$, and $(\mathcal{F}-\{F\}) \cup\left\{F-e_{1}\right\}$ is a covering family in $M_{0} / e_{1}$. By minimality, $M_{0} / e_{1}$ is a fan-extension of $N$, and therefore, so is $M_{0}$. Hence $M_{0} / e_{1}$ is not 3 -connected. As $M_{0} / e_{2}$ contains a parallel pair, it is not 3 -connected. The dual of Tutte's Triangle Lemma (see [9, Lemma 8.7.7]) implies there is a triangle, $T$, of $M_{0}$ containing $e_{1}$ and either $e_{2}$ or $e_{3}$. It follows from Proposition 5.6 that $T$ cannot be contained in $F$, for otherwise $\left\{e_{1}, e_{2}, e_{3}\right\}$ is a triad and a triangle. Let $f$ be the element in $T-\left\{e_{1}, e_{2}, e_{3}\right\}$. Then $N$ is a minor of $M_{0} / e_{1} \backslash f$, by 6.11.2. Since $\mathcal{F}$ is a covering family, there is a fan, $F_{f} \in \mathcal{F}$, such that $f$ is in $F_{f}$. As $F_{f}$ contains at least three elements of $E(N)$, any internal element of $F_{f}$ is contained in a triad. Orthogonality with the triangle $T$ now implies that $f$ is a terminal spoke element of $F_{f}$. Clearly $F_{f}-f$ is a fan of $M_{0}$.

If $e_{2}$ is in $T$, then $F+f=\left(f, e_{1}, \ldots, e_{n}\right)$ is a fan of $M_{0}$, and it is straightforward to see that $\left(\mathcal{F}-\left\{F, F_{f}\right\}\right) \cup\left\{F+f, F_{f}-f\right\}$ is a covering family of $M_{0}$. But now $e_{1}$ is an internal element of $F+f$ that is not in $E(N)$, contradicting 6.11.2. Hence $T=\left\{f, e_{1}, e_{3}\right\}$. This means that $n=4$, for otherwise $T$ violates orthogonality with the triad $\left\{e_{3}, e_{4}, e_{5}\right\}$. Thus $\left(e_{2}, e_{3}, e_{4}\right)$ or its reversal is in $\mathcal{F}_{N}$.

Let $F_{f}=\left(f_{1}, \ldots, f_{m}\right)$, where $f=f_{1}$. By applying the duals of the previous arguments, we see that $\left\{f_{1}, f_{3}\right\}$ is contained in a triad of $M_{0}$, that $m=4$, and that either $\left(f_{2}, f_{3}, f_{4}\right)$ or its reversal is in $\mathcal{F}_{N}$. The triad containing $\left\{f_{1}, f_{3}\right\}$ contains either $e_{1}$ or $e_{3}$, by orthogonality with $T$, and the latter case cannot occur, by orthogonality with $\left\{e_{2}, e_{3}, e_{4}\right\}$. Thus $\left\{f_{3}, f_{1}, e_{1}\right\}$ is a triad, and $\left(f_{4}, f_{2}, f_{3}, f_{1}, e_{1}, e_{3}, e_{2}, e_{4}\right)$ is a fan of $M_{0}$.

Let $I$ and $I^{*}$ be, respectively, independent and coindependent sets such that $I \cap I^{*}=\emptyset$ and $N=M_{0} / I \backslash I^{*}$. Let $N^{\prime}=M_{0} /\left(I-\left\{e_{1}, f_{1}\right\}\right) \backslash\left(I^{*}-\left\{e_{1}, f_{1}\right\}\right)$. Note that $\left\{f_{4}, f_{2}, f_{3}\right\}$ and $\left\{e_{3}, e_{2}, e_{4}\right\}$ are, respectively, a triad and a triangle in $N^{\prime}$, for otherwise $N$ contains a circuit or cocircuit with at most two elements. Since $\left\{f_{2}, f_{3}, f_{1}\right\}$ is a union of circuits in $N^{\prime}$, it is easy to see it is a circuit of $N^{\prime}$, for otherwise we can use orthogonality with $\left\{f_{4}, f_{2}, f_{3}\right\}$ to show that $\left\{f_{2}, f_{3}\right\}$ contains a circuit in $N^{\prime}$, and hence in $N$. Hence $f_{1}$ is not a loop of $N^{\prime}$. Similarly, $\left\{e_{1}, e_{3}, e_{2}\right\}$ is a triad in $N^{\prime}$ and $e_{1}$ is not a coloop. Since $\left\{f_{3}, f_{1}, e_{1}\right\}$ is a union of cocircuits, orthogonality with $\left\{f_{2}, f_{3}, f_{1}\right\}$ now implies that $\left\{f_{3}, f_{1}, e_{1}\right\}$ is a triad in $N^{\prime}$. Orthogonality with $\left\{e_{1}, e_{3}, e_{2}\right\}$ implies $\left\{f_{1}, e_{1}, e_{3}\right\}$ is a triangle in $N^{\prime}$. Therefore $\left(f_{4}, f_{2}, f_{3}, f_{1}, e_{1}, e_{3}, e_{2}, e_{4}\right)$ is a fan of $N^{\prime}$.

Because $N^{\prime} \backslash e_{1}$ contains the codependent triangle $\left\{f_{1}, f_{3}, f_{2}\right\}$, we see that $N$ is not a minor of $N^{\prime} \backslash e_{1}$. Therefore it is a minor of $N^{\prime} / e_{1}$, and this matroid contains the parallel pair $\left\{f_{1}, e_{3}\right\}$, so $N=N^{\prime} / e_{1} \backslash f_{1}$. By applying Proposition 6.9 to the fan $\left(e_{3}, e_{1}, f_{1}, f_{3}\right)$, we see that $N^{\prime}$ is 3-connected. Since $|E(M)|-|E(N)|>2=\left|E\left(N^{\prime}\right)\right|-|E(N)|, N^{\prime}$ is a proper minor of $M_{0}$. Therefore $N^{\prime}$ is a fan-extension of $N$.

As $\left|E\left(N^{\prime}\right)\right|-|E(N)|=2$, it follows that $N^{\prime}$ is obtained from $N$ by either one or two fan-lengthening moves. However, none of $N^{\prime} / e_{1}, N^{\prime} \backslash e_{1}, N^{\prime} / f_{1}, N^{\prime} \backslash f_{1}$ is 3-connected, 
so $N^{\prime}$ is obtained from $N$ by a single fan-lengthening move. Hence there is a covering family, $\mathcal{F}^{\prime}$, of $N^{\prime}$ containing a fan, $F^{\prime}$, such that $e_{1}$ and $f_{1}$ are consecutive elements of $F^{\prime}$. Assume that $e_{3}$ is in $F^{\prime}$. Because $\mathcal{F}^{\prime}$ is a covering family and $\left(e_{2}, e_{3}, e_{4}\right)$ or its reversal is in $\mathcal{F}_{N}$, it follows that $\left(e_{2}, e_{3}, e_{4}\right)$ is consistent with $F^{\prime}$. This means that $F^{\prime}$ contains $\left\{f_{1}, e_{1}, e_{3}, e_{2}, e_{4}\right\}$. By applying Proposition 5.6 to the triangle $\left\{f_{1}, e_{1}, e_{3}\right\}$, the triad $\left\{e_{1}, e_{3}, e_{2}\right\}$, and the triangle $\left\{e_{3}, e_{2}, e_{4}\right\}$, we see that $\left(f_{1}, e_{1}, e_{3}, e_{2}, e_{4}\right)$ is a contiguous subsequence of $F^{\prime}$. Hence $\left(e_{2}, e_{3}, e_{4}\right)$ is not consistent with $F^{\prime}$ after all. This contradiction shows that $e_{3}$ is not in $F^{\prime}$. As $\left\{e_{3}, e_{1}, f_{1}\right\}$ is a triangle, Proposition 5.3 implies that, up to reversing, $e_{1}$ and $f_{1}$ are the first two elements in $F^{\prime}$, and $F^{\prime}$ starts with a rim element. If $f_{3}$ is not in $F^{\prime}$, then we can apply Proposition 5.3 to the triad $\left\{f_{3}, f_{1}, e_{1}\right\}$ and get the contradiction that $F^{\prime}$ starts with a spoke element. Therefore $f_{3}$ is in $F^{\prime}$, and therefore $\left(f_{2}, f_{3}, f_{4}\right)$ is consistent with $F^{\prime}$. As $\left\{e_{1}, f_{1}, f_{3}\right\},\left\{f_{1}, f_{3}, f_{2}\right\}$, and $\left\{f_{3}, f_{2}, f_{4}\right\}$ are triangles or triads of $N^{\prime}$ contained in $F^{\prime}$, Proposition 5.6 implies that $\left(e_{1}, f_{1}, f_{3}, f_{2}, f_{4}\right)$ is a contiguous subsequence of $F^{\prime}$, so $\left(f_{2}, f_{3}, f_{4}\right)$ is not consistent with $F^{\prime}$ after all. This contradiction completes the proof.

Lemma 6.12. Let $M_{0}$ be a matroid isomorphic to $M$ such that $N$ is a minor of $M_{0}$, but $M_{0}$ is not a fan-extension of $N$. Assume that $\left(e_{1}, \ldots, e_{n}\right)$ is a fan of $M_{0}$, where $n \geqslant 5$, and $\left\{e_{1}, e_{2}, e_{3}\right\}$ is a triangle. If $N$ is a minor of $M_{0} / e_{2} \backslash e_{3}$ and some ordering of $\left\{e_{1}, e_{4}, \ldots, e_{n}\right\}$ is in $\mathcal{F}_{N}$, then either $\left(e_{1}, e_{4}, \ldots, e_{n}\right)$ or its reversal is in $\mathcal{F}_{N}$.

Proof. We assume that some ordering of $\left\{e_{1}, e_{4}, \ldots, e_{n}\right\}$ is in $\mathcal{F}_{N}$. Let $I$ and $I^{*}$ be independent and coindependent sets such that $I \cap I^{*}=\emptyset$ and $N=M_{0} / I \backslash I^{*}$ and let $N^{\prime}=M_{0} /\left(I-\left\{e_{2}, e_{3}\right\}\right) \backslash\left(I^{*}-\left\{e_{2}, e_{3}\right\}\right)$. If $4 \leqslant i \leqslant n-2$ and $i$ is odd (respectively, even), then $\left\{e_{i}, e_{i+1}, e_{i+2}\right\} \subseteq E(N)$, and $\left\{e_{i}, e_{i+1}, e_{i+2}\right\}$ is a circuit (cocircuit) in $M_{0}$, and is therefore a union of circuits (cocircuits) in $N^{\prime}$. Hence it is a circuit (cocircuit) in $N^{\prime}$, or else $N$ contains a circuit (cocircuit) of at most two elements, which contradicts the 3 -connectivity of $N$. Thus $\left(e_{4}, \ldots, e_{n}\right)$ is a fan of $N^{\prime}$. As $\left\{e_{3}, e_{4}, e_{5}\right\}$ is a union of circuits in $N^{\prime}$, it is a circuit, for otherwise $\left\{e_{4}, e_{5}\right\}$ contains a circuit in $N^{\prime}$, and hence in $N$. Since $\left\{e_{1}, e_{2}, e_{3}\right\}$ is a union of circuits in $N^{\prime}, e_{2}$ is not a coloop of $N^{\prime}$. Now, as $\left\{e_{2}, e_{3}, e_{4}\right\}$ is a union of cocircuits, orthogonality with $\left\{e_{3}, e_{4}, e_{5}\right\}$ shows that $\left\{e_{2}, e_{3}, e_{4}\right\}$ is a triad of $N^{\prime}$. Finally, $e_{1}$ is not a loop of $N$, and hence not a loop in $N^{\prime}$. Neither $e_{2}$ nor $e_{3}$ is a loop in $N^{\prime}$, as $\left\{e_{2}, e_{3}, e_{4}\right\}$ is a triad. If $\left\{e_{1}, e_{2}, e_{3}\right\}$ is not a circuit, then it is contained in a parallel class, and this leads to a contradiction to orthogonality with $\left\{e_{2}, e_{3}, e_{4}\right\}$. Thus we have shown $\left(e_{1}, \ldots, e_{n}\right)$ is a fan in $N^{\prime}$.

As $N^{\prime} \backslash e_{2}$ contains a codependent triangle, it cannot have $N$ as a minor. Therefore $N^{\prime} / e_{2}$ has $N$ as a minor, as well as the parallel pair $\left\{e_{1}, e_{3}\right\}$. Therefore $N=N^{\prime} / e_{2} \backslash e_{3}$. Proposition 6.9 implies that $N^{\prime}$ is 3-connected. As $\left|E\left(M_{0}\right)\right|-|E(N)|>2=\left|E\left(N^{\prime}\right)\right|-$ $|E(N)|$, we see that $N^{\prime}$ is a fan-extension of $N$.

Let $F^{\prime}$ be the fan in a covering family of $N^{\prime}$ such that some ordering of $\left\{e_{1}, e_{4}, \ldots, e_{n}\right\}$ is consistent with $F^{\prime}$. As $\left(e_{1}, \ldots, e_{n}\right)$ is a fan of $N^{\prime}$ that intersects $F^{\prime}$ in at least three elements, including $e_{1}$ and $e_{n}$, we see from Lemma 5.8 that there is a contiguous subsequence of $F^{\prime}$ comprising the elements $\left\{e_{1}, \ldots, e_{n}\right\}$. Now, repeatedly applying Proposition 5.6, it follows that $\left(e_{1}, \ldots, e_{n}\right)$ is a contiguous subsequence of $F^{\prime}$. Thus $\left(e_{1}, e_{4}, \ldots, e_{n}\right)$ is consis- 
tent with $F^{\prime}$, so this fan, or its reversal, is in $\mathcal{F}_{N}$.

If we apply an arbitrary permutation to the element labels of $M$, we do not know if the resulting matroid will be a fan-extension of $N$, or, indeed, if it will have $N$ as a minor. In the next lemma, and in Lemma 6.15, we look for circumstances under which the permuted matroid is guaranteed to have $N$ as a minor, but not to be a fan-extension of $N$. This will allow us to relabel elements of $M$ without losing generality.

Lemma 6.13. Let $M_{0}$ be isomorphic to $M$, and assume that $M_{0}$ has $N$ as a minor, but is not a fan-extension of $N$. Assume that $\left(e_{1}, e_{2}, e_{3}, e_{4}\right)$ is a fan of $M_{0}$ with $e_{2}$ as a rim element, and $N$ is a minor of $M_{0} / e_{2} \backslash e_{3}$. Let $M^{\prime}$ be the matroid obtained from $M_{0}$ by swapping the labels on $e_{1}$ and $e_{3}$. Then $M^{\prime}$ contains $N$ as a minor, but is not a fan-extension of $N$.

Proof. Because $\left\{e_{1}, e_{3}\right\}$ is a parallel pair of $M_{0} / e_{2}$, it follows that $M^{\prime} / e_{2} \backslash e_{3}=M_{0} / e_{2} \backslash e_{3}$, so $N$ is a minor of $M^{\prime} / e_{2} \backslash e_{3}$. Proposition 6.4 implies $M_{0} / e_{2} \backslash e_{3}$, and hence $M^{\prime} / e_{2} \backslash e_{3}$, is 3 -connected. Note that $\left(e_{3}, e_{2}, e_{1}, e_{4}\right)$ is a fan of $M^{\prime}$, where $e_{2}$ is a rim element. Assume for a contradiction that $M^{\prime}$ is a fan-extension of $N$. As $e_{2}, e_{3} \notin E(N)$, there is a covering family of $M^{\prime}$ that contains fans that contain $e_{2}$ and $e_{3}$.

6.13.1. If $\mathcal{F}^{\prime}$ is a covering family of $M^{\prime}$, then no fan in $\mathcal{F}^{\prime}$ contains $\left\{e_{1}, e_{2}, e_{3}\right\}$.

Proof. Assume $F^{\prime} \in \mathcal{F}^{\prime}$ contains $\left\{e_{1}, e_{2}, e_{3}\right\}$. Then Proposition 5.6 implies that $\left\{e_{1}, e_{2}, e_{3}\right\}$ is a set of three consecutive elements in $F^{\prime}$. As $e_{2}, e_{3} \notin E(N)$, it now is obvious that we can swap the labels on $e_{1}$ and $e_{3}$ and obtain a covering family of $M_{0}$, contradicting Lemma 6.11.

6.13.2. Let $\mathcal{F}^{\prime}$ be a covering family of $M^{\prime}$ and let $F_{2}$ be a fan in $\mathcal{F}^{\prime}$ that contains $e_{2}$. Then $e_{2}$ is a terminal rim element of $F_{2}$.

Proof. Assume that $e_{2}$ is in a triangle, $T$, contained in $F_{2}$. If $e_{3}$ is not in $T$, then $M^{\prime} / e_{2} \backslash e_{3}$ contains a parallel pair, and this is impossible as $M^{\prime} / e_{2} \backslash e_{3}$ is 3 -connected with at least four elements in its ground set. Therefore $e_{2}, e_{3} \in T$. Orthogonality with the triad $\left\{e_{2}, e_{1}, e_{4}\right\}$ shows that $e_{1} \in T$ or $e_{4} \in T$. The former case is not true by 6.13 .1 , so $T=\left\{e_{2}, e_{3}, e_{4}\right\}$. As $\left\{e_{1}, e_{2}, e_{3}\right\}$ is also a triangle, $\left\{e_{1}, e_{2}, e_{3}, e_{4}\right\}$ is a $U_{2,4}$-restriction of $M^{\prime}$ that intersects the triad $\left\{e_{2}, e_{1}, e_{4}\right\}$, contradicting Corollary 6.3. Therefore $e_{2}$ is contained in no triangle in $F_{2}$. Note that $F_{2}$ contains a fan in $\mathcal{F}_{N}$, as well as the element $e_{2}$, so it contains at least four elements. Therefore $e_{2}$ is a terminal rim element in $F_{2}$, as desired.

6.13.3. There is a covering family, $\mathcal{F}^{\prime}$, of $M^{\prime}$, and a fan $F^{\prime} \in \mathcal{F}^{\prime}$, such that $e_{2}$ and $e_{3}$ are consecutive elements in $F^{\prime}$.

Proof. Assume that this is not true. Let $\mathcal{F}^{\prime}$ be an arbitrary covering family of $M^{\prime}$. Let $F_{2}=\left(f_{1}, \ldots, f_{m}\right)$ be a fan in $\mathcal{F}^{\prime}$ where $e_{2}=f_{1}$ and $\left\{f_{1}, f_{2}, f_{3}\right\}$ is a triad. Orthogonality with the triangle $\left\{e_{1}, e_{2}, e_{3}\right\}$ shows that $e_{3}$ or $e_{1}$ is in $\left\{f_{2}, f_{3}\right\}$. Assume that $e_{3}$ is in $\left\{f_{2}, f_{3}\right\}$, so $e_{1} \notin F_{2}$ by 6.13.1. Because we have assumed that $e_{2}$ and $e_{3}$ are not consecutive in $F_{2}$, we deduce that $e_{3}=f_{3}$. Now $m \leqslant 4$, or else we have a contradiction to orthogonality 
between $\left\{e_{1}, e_{2}, e_{3}\right\}=\left\{e_{1}, f_{1}, f_{3}\right\}$ and the triad $\left\{f_{3}, f_{4}, f_{5}\right\}$. However, $F_{2}$ contains a fan in $\mathcal{F}_{N}$ as well as the elements $e_{2}$ and $e_{3}$, so $m \geqslant 5$. This contradiction shows that $e_{1}$ is in $\left\{f_{2}, f_{3}\right\}$, and therefore $e_{3} \notin F_{2}$.

Now $\left\{f_{1}, f_{2}, f_{3}\right\}=\left\{e_{1}, e_{2}, z\right\}$ for some element $z$, where $z \neq e_{3}$. If $z \neq e_{4}$, then $\left\{e_{2}, e_{1}, e_{4}, z\right\}$ is a $U_{2,4}$-corestriction of $M^{\prime}$ that intersects the triangle $\left\{e_{1}, e_{2}, e_{3}\right\}$, contradicting the dual of Corollary 6.3. Therefore $z=e_{4}$, so $\left\{f_{1}, f_{2}, f_{3}\right\}=\left\{e_{1}, e_{2}, e_{4}\right\}$. If $\left(f_{1}, f_{2}, f_{3}\right)=\left(e_{2}, e_{1}, e_{4}\right)$, then $F_{2}+e_{3}=\left(e_{3}, f_{1}, \ldots, f_{m}\right)$ is a fan of $M^{\prime}$. Let $F_{3}$ be the fan in $\mathcal{F}^{\prime}$ that contains $e_{3}$. Then $F_{3}$ contains a fan in $\mathcal{F}_{N}$ as well as $e_{3}$, so $\left|F_{3}\right| \geqslant 4$. If $e_{3}$ is an internal element in $F_{3}$, then it is contained in a triad that is contained in $F_{3}$, and such a triad violates orthogonality with $\left\{e_{1}, e_{2}, e_{3}\right\}$. Therefore $e_{3}$ is a terminal spoke element of $F_{3}$. Now we easily see that $\left(\mathcal{F}^{\prime}-\left\{F_{2}, F_{3}\right\}\right) \cup\left\{F_{2}+e_{3}, F_{3}-e_{3}\right\}$ is a covering family of $M^{\prime}$, and $e_{3}$ and $e_{2}$ are consecutive in $F_{2}+e_{3}$. This contradicts our assumption, so $\left(f_{1}, f_{2}, f_{3}\right)=\left(e_{2}, e_{4}, e_{1}\right)$.

Now $m \leqslant 4$, or else we have a contradiction to orthogonality between the triangle $\left\{e_{1}, e_{2}, e_{3}\right\}$ and the triad $\left\{f_{3}, f_{4}, f_{5}\right\}$ (recall $e_{3}$ is not in $F_{2}$ ). In fact, $m=4$, since $F_{2}$ contains one of the fans in $\mathcal{F}_{N}$, as well as $e_{2}$. Therefore $F_{2}=\left(f_{1}, f_{2}, f_{3}, f_{4}\right)=\left(e_{2}, e_{4}, e_{1}, f_{4}\right)$, and $\left(e_{4}, e_{1}, f_{4}\right)$ or $\left(f_{4}, e_{1}, e_{4}\right)$ is in $\mathcal{F}_{N}$. However, $\left(e_{3}, f_{1}, f_{3}, f_{2}, f_{4}\right)=\left(e_{3}, e_{2}, e_{1}, e_{4}, f_{4}\right)$ is also a fan of $M^{\prime}$, so $\left(e_{1}, e_{2}, e_{3}, e_{4}, f_{4}\right)$ is a fan of $M_{0}$. Now $\left\{e_{1}, e_{2}, e_{3}\right\}$ is a triangle, and $N$ is a minor of $M_{0} / e_{2} \backslash e_{3}$. By Lemma $6.12,\left(e_{1}, e_{4}, f_{4}\right)$ or $\left(f_{4}, e_{4}, e_{1}\right)$ is in $\mathcal{F}_{N}$. Thus $\mathcal{F}_{N}$ contains two distinct fans that are non-disjoint. This is a contradiction.

By 6.13.2 and 6.13.3 we can let $\mathcal{F}^{\prime}$ be a covering family of $M^{\prime}$ and let $F^{\prime}=\left(f_{1}, \ldots, f_{m}\right)$ in $\mathcal{F}^{\prime}$ be such that $\left(f_{1}, f_{2}\right)=\left(e_{2}, e_{3}\right)$ where $m \geqslant 5$ and $\left\{f_{1}, f_{2}, f_{3}\right\}$ is a triad. Now 6.13 .1 implies $e_{1} \notin F^{\prime}$. Observe that $F^{\prime}+e_{1}=\left(e_{1}, f_{1}, f_{2}, \ldots, f_{m}\right)$ is a fan of $M^{\prime}$. By 6.13.1, $\left(\mathcal{F}^{\prime}-\left\{F^{\prime}\right\}\right) \cup\left\{F^{\prime}+e_{1}\right\}$ cannot be a covering family in $M^{\prime}$. Therefore there is a fan, $G=\left(g_{1}, \ldots, g_{t}\right)$, in $\mathcal{F}^{\prime}-\left\{F^{\prime}\right\}$, that contains $e_{1}$. Orthogonality with $\left\{e_{1}, e_{2}, e_{3}\right\}$ shows that $e_{1}$ is not contained in a triad that is contained in $G$. Let $G_{N}$ be the fan in $\mathcal{F}_{N}$ that is consistent with $G$. If $e_{1}$ is not in $G_{N}$, then $G$ contains at least four elements, so $e_{1}$ must be a terminal element of $G$. In this case, it is easy to see that $\left(\mathcal{F}^{\prime}-\left\{F^{\prime}, G\right\}\right) \cup\left\{F^{\prime}+e_{1}, G-e_{1}\right\}$ is a covering family of $M^{\prime}$, which leads to a contradiction with 6.13.1. Therefore $e_{1}$ is contained in $G_{N}$.

Assume that $t=3$. Then, by reversing, we can assume that $\left(g_{1}, g_{2}, g_{3}\right)$ is in $\mathcal{F}_{N}$. Orthogonality with $\left\{e_{1}, e_{2}, e_{3}\right\}$ implies $\left\{g_{1}, g_{2}, g_{3}\right\}$ is a triangle. But $\left\{e_{2}, e_{1}, e_{4}\right\}$ is a triad in $M^{\prime}$, so orthogonality requires that $e_{4}$ is in $\left\{g_{1}, g_{2}, g_{3}\right\}$. Let $g$ be the element in $\left\{g_{1}, g_{2}, g_{3}\right\}-$ $\left\{e_{1}, e_{4}\right\}$. Then $\left(e_{3}, e_{2}, e_{1}, e_{4}, g\right)$ is a fan in $M^{\prime}$, so $\left(e_{1}, e_{2}, e_{3}, e_{4}, g\right)$ is a fan in $M_{0}$. As $N$ is a minor of $M_{0} / e_{2} \backslash e_{3}$, Lemma 6.12 implies that, up to reversing $\left(e_{1}, e_{4}, g\right)$ is in $\mathcal{F}_{N}$, so $\left(e_{1}, e_{4}, g\right)=\left(g_{1}, g_{2}, g_{3}\right)$. This means that $\left(e_{3}, e_{2}, e_{1}, e_{4}, g\right)$ is a fan in $M^{\prime}$ that is consistent with $\left(g_{1}, g_{2}, g_{3}\right)$, and

$$
\left(\mathcal{F}^{\prime}-\left\{F^{\prime},\left(e_{1}, e_{4}, g\right)\right\}\right) \cup\left\{F^{\prime}-\left\{f_{1}, f_{2}\right\},\left(e_{3}, e_{2}, e_{1}, e_{4}, g\right)\right\}
$$

is a covering family in $M^{\prime}$, contradicting 6.13.1. Therefore $t \geqslant 4$.

Because $e_{1}$ is not contained in any triad that is contained in $G$, we see that $e_{1}$ is a terminal spoke element in $\left(g_{1}, \ldots, g_{t}\right)$. By reversing as necessary, we can assume that $e_{1}=g_{1}$ and $\left\{g_{1}, g_{2}, g_{3}\right\}$ is a triangle. 
Orthogonality between $\left\{g_{1}, g_{2}, g_{3}\right\}$ and $\left\{e_{1}, e_{2}, e_{4}\right\}$ requires that $e_{4}$ is in $\left\{g_{2}, g_{3}\right\}$. Assume that $e_{4}=g_{2}$. Then

$$
G^{\prime}=\left(e_{3}, e_{2}, g_{1}, g_{2}, g_{3}, \ldots, g_{t}\right)=\left(e_{3}, e_{2}, e_{1}, e_{4}, g_{3}, \ldots, g_{t}\right)
$$

is a fan in $M^{\prime}$. It is easy to see that $\left(\mathcal{F}^{\prime}-\left\{F^{\prime}, G\right\}\right) \cup\left\{F^{\prime}-\left\{f_{1}, f_{2}\right\}, G^{\prime}\right\}$ is a covering family in $M^{\prime}$, contradicting 6.13.1. Therefore $g_{3}=e_{4}$.

Next we observe that $t=4$, for otherwise $\left\{g_{3}, g_{4}, g_{5}\right\}$ is a triangle and $\left\{e_{1}, e_{2}, e_{4}\right\}$ is a triad that intersects it in the element $e_{4}=g_{3}$. Now $G_{N}$ is a subsequence of at least three elements from $\left(g_{1}, g_{2}, g_{3}, g_{4}\right)$ or $\left(g_{4}, g_{3}, g_{2}, g_{1}\right)$ that contains $e_{1}$.

Assume that $g_{4}$ is not in $G_{N}$. Then $G_{N}$ is equal, up to reversing, to $\left(g_{1}, g_{2}, g_{3}\right)$. Since $\left(e_{3}, e_{2}, g_{1}, g_{3}, g_{2}\right)=\left(e_{3}, e_{2}, e_{1}, e_{4}, g_{2}\right)$ is a fan in $M^{\prime},\left(e_{1}, e_{2}, e_{3}, e_{4}, g_{2}\right)$ is a fan in $M_{0}$. As $N$ is a minor of $M_{0} / e_{2} \backslash e_{3}$, we see from Lemma 6.12 that $\left(e_{1}, e_{4}, g_{2}\right)=\left(g_{1}, g_{3}, g_{2}\right)$, or its reversal, is in $\mathcal{F}_{N}$. Therefore $\mathcal{F}_{N}$ contains two distinct fans of three elements that are non-disjoint. This contradiction shows that $g_{4}$ is in $G_{N}$.

Assume that exactly one of $g_{2}$ and $g_{3}$ is in $G_{N}$. Then $G_{N}$ is consistent with the fan $G^{\prime}=\left(e_{3}, e_{2}, g_{1}, g_{3}, g_{2}, g_{4}\right)=\left(e_{3}, e_{2}, e_{1}, e_{4}, g_{2}, g_{4}\right)$ in $M^{\prime}$, and

$$
\left(\mathcal{F}^{\prime}-\left\{F^{\prime}, G\right\}\right) \cup\left\{F^{\prime}-\left\{f_{1}, f_{2}\right\}, G^{\prime}\right\}
$$

is a covering family of $M^{\prime}$, violating 6.13 .1 . We conclude that $G_{N}$ is equal, up to reversing, to $\left(g_{1}, g_{2}, g_{3}, g_{4}\right)$.

Now $\left(e_{3}, e_{2}, g_{1}, g_{3}, g_{2}, g_{4}\right)=\left(e_{3}, e_{2}, e_{1}, e_{4}, g_{2}, g_{4}\right)$ is a fan in $M^{\prime}$, so $\left(e_{1}, e_{2}, e_{3}, e_{4}, g_{2}, g_{4}\right)$ is a fan in $M_{0}$, and some ordering of $\left\{e_{1}, e_{4}, g_{2}, g_{4}\right\}$ is in $\mathcal{F}_{N}$. By Lemma $6.12,\left(e_{1}, e_{4}, g_{2}, g_{4}\right)=$ $\left(g_{1}, g_{3}, g_{2}, g_{4}\right)$ or its reversal is in $\mathcal{F}_{N}$. This shows that $\mathcal{F}_{N}$ contains two distinct fans that are non-disjoint. We have a contradiction that completes the proof of the lemma.

Now we have some control over the ways in which we may permute the element labels on $M$. The next result shows a consequence of this: we can assume that there is an element in $M$ whose removal is 3 -connected with $N$ as a minor.

Lemma 6.14. There exists a matroid $M_{0}$, isomorphic to $M$, such that $N$ is a minor of $M_{0}$, but $M_{0}$ is not a fan-extension of $N$. Moreover, $M_{0}$ has a 3-connected single-element deletion or contraction that has $N$ as a minor.

Proof. We will assume that the lemma is false, so that no such matroid $M_{0}$ exists. Let $e$ be an element in $E(M)-E(N)$. We use Proposition 6.8. By possibly replacing $M$, $N$, and $\mathcal{M}$ with their duals, we can assume that $\left(e_{1}, \ldots, e_{n}\right)$ is a fan of $M$ such that $N$ is a minor of $M / e_{i} \backslash e_{i+1}$ for some rim element $e_{i}$, where $i \in\{2, \ldots, n-2\}$. Moreover, $M / e_{i} \backslash e_{i+1}$ is 3 -connected. We assume that amongst all such fans, $\left(e_{1}, \ldots, e_{n}\right)$ has been chosen so that $n$ is as large as possible.

6.14.1. If $e_{1}$ is a spoke element of $\left(e_{1}, \ldots, e_{n}\right)$, then $M \backslash e_{1}$ is 3-connected, and if $e_{1}$ is a rim element, then $M / e_{1}$ is 3-connected. 
Proof. Assume $e_{1}$ is a rim element. Then $n>4$, for otherwise $i=2$, and both $e_{1}$ and $e_{2}$ are rim elements. If $M / e_{1}$ is not 3-connected, then $e_{1}$ is in a triangle. It follows from Proposition 5.6 that this triangle is not contained in $\left\{e_{1}, \ldots, e_{n}\right\}$. Let $z \notin\left\{e_{1}, \ldots, e_{n}\right\}$ be an element in a triangle with $e_{1}$. Orthogonality with $\left\{e_{1}, e_{2}, e_{3}\right\}$ and $\left\{e_{3}, e_{4}, e_{5}\right\}$ shows $\left\{e_{1}, e_{2}, z\right\}$ is a triangle, and $\left(z, e_{1}, \ldots, e_{n}\right)$ is a fan. This contradicts the maximality of $n$. Similarly, if $e_{1}$ is a spoke element and $M \backslash e_{1}$ is not 3-connected, then $e_{1}$ is in a triad with an element $z \notin\left\{e_{1}, \ldots, e_{n}\right\}$, and either $\left(z, e_{1}, \ldots, e_{n}\right)$ is a fan, or $n=4$, and $\left\{e_{1}, e_{3}, z\right\}$ is a triad. In the first case we have a contradiction to the maximality of $n$. In the latter case, $n=4$ and $i=2$, so $N$ is a minor of $M / e_{2} \backslash e_{3}$, but $\left\{e_{1}, e_{3}, z\right\}$ is a triad that contains the parallel pair $\left\{e_{1}, e_{3}\right\}$ in $M / e_{2}$, contradicting Proposition 6.2.

Define $M^{(0)}$ to be $M$. We obtain $M^{(1)}$ from $M^{(0)}$ by swapping labels on $e_{i-1}$ and $e_{i+1}$. Then $M^{(1)} / e_{i} \backslash e_{i+1}=M^{(0)} / e_{i} \backslash e_{i+1}$, and by applying Lemma 6.13 to $\left(e_{i-1}, e_{i}, e_{i+1}, e_{i+2}\right)$ we see that $M^{(1)}$ is not a fan-extension of $N$. Let $M^{(2)}$ be obtained from $M^{(1)}$ by swapping the labels on $e_{i-2}$ and $e_{i}$. Then $M^{(2)} / e_{i} \backslash e_{i+1}=M^{(1)} / e_{i} \backslash e_{i+1}$, and the dual of Lemma 6.13 implies $M^{(2)}$ is not a fan-extension of $N$. In general, when $j \in\{1, \ldots, i-1\}$, we obtain $M^{(j)}$ from $M^{(j-1)}$ by swapping labels on $e_{i-j}$ and $e_{i+1}$ if $j$ is odd, and on $e_{i-j}$ and $e_{i}$ if $j$ is even. An obvious inductive argument establishes the following statement.

6.14.2. For every $j \in\{0,1, \ldots, i-1\}, M^{(j)} / e_{i} \backslash e_{i+1}=M / e_{i} \backslash e_{i+1}$, so $M^{(j)} / e_{i} \backslash e_{i+1}$ has $N$ as a minor. Moreover, $M^{(j)}$ is not a fan-extension of $N$.

Note that $M^{(i-1)}$ is obtained from $M$ by relabeling $\left(e_{1}, \ldots, e_{n}\right)$ as

$$
\begin{cases}\left(e_{i}, e_{i+1}, e_{1}, \ldots, e_{i-1}, e_{i+2}, \ldots, e_{n}\right) & \text { if } i \text { is odd } \\ \left(e_{i+1}, e_{i}, e_{1}, \ldots, e_{i-1}, e_{i+2}, \ldots, e_{n}\right) & \text { if } i \text { is even }\end{cases}
$$

Assume $i$ is odd, so $e_{1}$ is a rim element of $\left(e_{1}, \ldots, e_{n}\right)$ in $M$. There is an isomorphism from $M$ to $M^{(i-1)}$ that relabels $e_{1}$ as $e_{i}$. Therefore $M^{(i-1)} / e_{i}$ is 3-connected, by 6.14.1. Similarly, if $i$ is even, then $e_{1}$ is a spoke element and $M^{(i-1)} \backslash e_{i+1}$ is 3-connected. In either case, $M^{(i-1)}$ is isomorphic to $M$, and has $N$ as a minor, but is not a fan-extension of $N$. Since $M^{(i-1)}$ has a 3-connected single-element deletion or contraction that has $N$ as a minor, the lemma is proved.

Now we can assume $M$ has a 3-connected single-element deletion or contraction with $N$ as a minor. Lemma 6.13 considered swapping labels on elements that belonged to a fan of $M$. In the next lemma we swap labels on elements that belong to a fan in a 3-connected single-element deletion.

Lemma 6.15. Let $M_{0}$ be isomorphic to $M$, and assume that $M_{0}$ has $N$ as a minor, but is not a fan-extension of $N$. Assume $M_{0} \backslash e$ is 3-connected and has $\left(e_{1}, e_{2}, e_{3}, e_{4}\right)$ as a fan. Moreover, assume that either:

(i) $e_{2}$ is a rim element and $N$ is a minor of $M_{0} \backslash e / e_{2} \backslash e_{3}$, or

(ii) $e_{2}$ is a spoke element, and $N$ is a minor of $M_{0} \backslash e \backslash e_{2} / e_{3}$. 
Let $M^{\prime}$ be obtained from $M_{0}$ by swapping the labels on $e_{1}$ and $e_{3}$. Then $M^{\prime}$ has $N$ as a minor, but is not a fan-extension of $N$.

Proof. Note that in case (i), $M^{\prime} \backslash e / e_{2} \backslash e_{3}=M_{0} \backslash e / e_{2} \backslash e_{3}$, and in case (ii), $M^{\prime} \backslash e \backslash e_{2} / e_{3}=$ $M_{0} \backslash e \backslash e_{2} / e_{3}$, so $M^{\prime}$ certainly has $N$ as a minor. If $\left(e_{1}, e_{2}, e_{3}, e_{4}\right)$ is a fan of $M_{0}$, then we could apply Lemma 6.13 or its dual to $\left(e_{1}, e_{2}, e_{3}, e_{4}\right)$, and deduce that $M^{\prime}$ is not a fanextension of $N$. In this case there is nothing left to prove, so we assume that $\left(e_{1}, e_{2}, e_{3}, e_{4}\right)$ is not a fan of $M_{0}$. Thus, if statement (i) holds, $\left\{e, e_{2}, e_{3}, e_{4}\right\}$ is a cocircuit of $M_{0}$. In this case we set $\left(x_{1}, x_{2}, x_{3}, x_{4}\right)$ to be $\left(e_{1}, e_{2}, e_{3}, e_{4}\right)$. If statement (ii) holds, $\left\{e, e_{1}, e_{2}, e_{3}\right\}$ is a cocircuit, and in this case we set $\left(x_{1}, x_{2}, x_{3}, x_{4}\right)$ to be $\left(e_{4}, e_{3}, e_{2}, e_{1}\right)$. In either case, $\left(x_{1}, x_{2}, x_{3}, x_{4}\right)$ is a fan of $M_{0} \backslash e$ with $x_{1}$ as a spoke element, $N$ is a minor of $M_{0} \backslash e / x_{2} \backslash x_{3}$, and $\left\{e, x_{2}, x_{3}, x_{4}\right\}$ is a cocircuit of $M_{0}$.

6.15.1. $M_{0} \backslash e / x_{2} \backslash x_{3}$ is 3-connected.

Proof. This follows by applying Proposition 6.4 to $M_{0} \backslash e$.

6.15.2. $M_{0} \backslash x_{3}$ is 3-connected.

Proof. Assume otherwise. Then $x_{3}$ is contained in a triad, $T^{*}$, of $M_{0}$. As $M_{0} \backslash e$ is 3-connected, $T^{*}$ is also a triad in $M_{0} \backslash e$. Proposition 6.2 and orthogonality with $\left\{x_{1}, x_{2}, x_{3}\right\}$ shows that $T^{*}$ contains exactly one of $x_{1}$ or $x_{2}$. If $x_{1}$ is in $T^{*}$, then $T^{*}$ is a dependent triad in $M_{0} / x_{2}$. Since $N$ is a minor of $M_{0} / x_{2}$ this contradicts Proposition 6.2. Therefore $x_{2}, x_{3} \in T^{*}$. Note $T^{*} \neq\left\{x_{2}, x_{3}, x_{4}\right\}$, as $\left\{e, x_{2}, x_{3}, x_{4}\right\}$ is a cocircuit of $M_{0}$. Therefore $T^{*} \cup x_{4}$ is a $U_{2,4}$-corestriction in $M_{0} \backslash e$ that intersects the triangle $\left\{x_{1}, x_{2}, x_{3}\right\}$. This contradiction to the dual of Corollary 6.3 completes the proof.

We will assume for a contradiction that $M^{\prime}$ is a fan-extension of $N$. Then there is a covering family of $M^{\prime}$ containing a fan, $F_{e}$, that contains $e$. As $M^{\prime} \backslash e$ is 3-connected (since it is isomorphic to $M_{0} \backslash e$ ) and $F_{e}$ contains at least four elements, it follows that $e$ is a terminal spoke element of a fan in $M_{0}$ that has at least four elements. Let $T_{e}$ be the triangle in this fan that contains $e$. Orthogonality with $\left\{e, x_{2}, x_{3}, x_{4}\right\}$ shows that $T_{e}$ contains $x_{2}, x_{3}$, or $x_{4}$. As $T_{e}-e$ is contained in a triad of $M_{0}$, it follows from 6.15.2 that $x_{3}$ is not in $T_{e}$. Assume that $x_{2}$ is in $T_{e}$. Orthogonality between $\left\{x_{1}, x_{2}, x_{3}\right\}$ and the triad containing $T_{e}-e$ shows that $x_{1}$ is contained in the triad. However, $x_{1}$ is not in $T_{e}$, for that would imply $T_{e} \cup x_{3}$ is a $U_{2,4}$-restriction of $M_{0}$ that intersects a triad, a contradiction. Now we can swap the labels on $x_{1}$ and $x_{3}$, and delete $x_{3}$. The resulting matroid has $N$ as a minor, since $x_{1}$ and $x_{3}$ are parallel in $M_{0} / x_{2}$, and contains the codependent triangle $T_{e}$. Because this is a contradiction, we conclude that $x_{2}$ is not in $T_{e}$, so $x_{4} \in T_{e}$. Let $z$ be the third element of $T_{e}$, so that $\left\{e, x_{4}, z\right\}$ is a triangle in $M_{0}$, and $\left\{x_{4}, z\right\}$ is contained in a triad, $\left\{x_{4}, z, z^{\prime}\right\}$, of $M_{0}$.

6.15.3. The elements in $\left\{e, x_{1}, x_{2}, x_{3}, x_{4}, z, z^{\prime}\right\}$ are pairwise distinct.

Proof. By the hypotheses of the lemma, $e, x_{1}, x_{2}, x_{3}$, and $x_{4}$ are pairwise distinct. Also, $e, x_{4}, z$, and $z^{\prime}$ are distinct members of a fan. If $z=x_{1}$, then orthogonality between $\left\{x_{1}, x_{2}, x_{3}\right\}$ and $\left\{x_{4}, z, z^{\prime}\right\}$ shows that $z^{\prime} \in\left\{x_{2}, x_{3}\right\}$. Then $\left\{x_{4}, z, z^{\prime}\right\}$ and $\left\{x_{2}, x_{3}, x_{4}\right\}$ are 
distinct triads of $M_{0} \backslash e$ that intersect in two elements. Hence $M_{0} \backslash e$ has a $U_{2,4}$-corestriction intersecting a triangle, a contradiction. If $z=x_{2}$, then $\left\{e, x_{2}, x_{4}\right\}$ is a triangle and a triad in $M_{0} \backslash x_{3}$, which is impossible by 6.15.2. Finally, if $z=x_{3}$, then $M_{0} \backslash x_{3}$ contains the series pair $\left\{x_{4}, z^{\prime}\right\}$, contradicting 6.15.2. This shows that $e, x_{1}, x_{2}, x_{3}, x_{4}$, and $z$ are pairwise distinct. If $z^{\prime} \in\left\{x_{1}, x_{2}, x_{3}\right\}$, then we contradict orthogonality with $\left\{x_{4}, z, z^{\prime}\right\}$. This completes the proof.

Now we subdivide into two cases.

Case 1. Statement (i) holds, so $M^{\prime}$ is obtained from $M_{0}$ by swapping the labels on $x_{1}$ and $x_{3}$. Since $M^{\prime}$ is a fan-extension of $N$, there is a covering family, $\mathcal{F}^{\prime}$, of $M^{\prime}$ containing a fan, $F_{2}$, that contains $x_{2}$. Let $F_{2}=\left(f_{1}, \ldots, f_{m}\right)$ Note that $m \geqslant 4$.

Assume that $x_{2}$ is contained in a triangle, $T_{2}$, that is contained in $F_{2}$. Suppose $x_{3}$ is not in $T_{2}$. From 6.15 .1 we see that $M^{\prime} \backslash e / x_{2} \backslash x_{3}$ contains no parallel pair, so $e$ is in $T_{2}$. This means $m \geqslant 5$, as $F_{2}$ contains $e, x_{2}$, and at least three elements of $E(N)$. Because $M^{\prime} \backslash e$ is 3-connected, $e$ is contained in no triad of $M^{\prime}$. Therefore we can assume that $e=f_{1}$, and $f_{1}$ is a spoke element of $F_{2}$. Thus $T_{2}=\left\{f_{1}, f_{2}, f_{3}\right\}$. As $N$ is a minor of $M^{\prime} / x_{2} \backslash e$, Proposition 6.7 implies $e$ and $x_{2}$ are consecutive in $F_{2}$, so $x_{2}=f_{2}$. Proposition 6.4 applied to $\left(f_{m}, \ldots, f_{1}\right)$ implies that $M^{\prime} / f_{2} \backslash f_{1}=M^{\prime} / x_{2} \backslash e$ is 3 -connected. This is impossible, as this matroid contains the parallel pair $\left\{x_{1}, x_{3}\right\}$. Therefore we conclude that $x_{3}$ is in $T_{2}$.

If $x_{1}$ is not in $T_{2}$, then $T_{2} \cup x_{1}$ is a $U_{2,4}$-restriction of $M^{\prime} \backslash e$, and $\left\{x_{2}, x_{1}, x_{4}\right\}$ is a triad. This contradiction shows that $T_{2}=\left\{x_{1}, x_{2}, x_{3}\right\}$. Now $\left\{x_{1}, x_{2}, x_{3}\right\}$ form a set of three consecutive elements in $F_{2}$, by Proposition 5.6. Let $F_{2}^{\prime}$ be the fan obtained from $F_{2}$ by swapping $x_{1}$ and $x_{3}$. As $x_{2}$ and $x_{3}$ are not in $E(N)$, it is clear that $\left(\mathcal{F}^{\prime}-\left\{F_{2}\right\}\right) \cup\left\{F_{2}^{\prime}\right\}$ is a covering family of $M_{0}$. This is a contradiction to Lemma 6.11, so we have to conclude that $x_{2}$ is contained in no triangle in $F_{2}$. Therefore we can assume that $x_{2}=f_{1}$, and this is a rim element of $F_{2}$.

By orthogonality with $\left\{x_{1}, x_{2}, x_{3}\right\}$, and the fact that $M^{\prime} \backslash x_{1}$ is 3-connected (by 6.15.2), we see that $\left\{f_{1}, f_{2}, f_{3}\right\}$ contains $x_{3}$, so $m \geqslant 5$. The dual of Proposition 6.7 shows that $x_{2}$ and $x_{3}$ are consecutive elements in $F_{2}$, so $x_{3}=f_{2}$. If $x_{1}$ is in $F_{2}$, then we can apply Proposition 5.6 to deduce that $x_{1}=f_{3}$. This implies $\left\{f_{1}, f_{2}, f_{3}\right\}$ is simultaneously a triad and a triangle of $M^{\prime}$, which is impossible. Hence $x_{1}$ is not in $F_{2}$. Let $\left(F_{2}+x_{1}\right)^{\prime}$ be the fan of $M_{0}$ obtained by appending $x_{1}$ to the end of $F_{2}$ and then swapping the locations of $x_{1}$ and $x_{3}$. Since $\left(\mathcal{F}^{\prime}-\left\{F_{2}\right\}\right) \cup\left(F_{2}+x_{1}\right)^{\prime}$ cannot be a covering family in $M_{0}$, it follows that $x_{1}$ is in another fan, $F_{1}$, belonging to $\mathcal{F}^{\prime}$. Orthogonality with $\left\{x_{1}, x_{2}, x_{3}\right\}$ shows that $x_{1}$ is a terminal spoke element in $F_{1}$. Because $\left(\mathcal{F}^{\prime}-\left\{F_{1}, F_{2}\right\}\right) \cup\left\{F_{1}-x_{1},\left(F_{2}+x_{1}\right)^{\prime}\right\}$ is not a covering family of $M_{0}$, we deduce that $x_{1}$ is in the fan of $\mathcal{F}_{N}$ that is consistent with $F_{1}$.

Let $T_{1}$ be the triangle in $F_{1}$ that contains $x_{1}$. Orthogonality with $\left\{e, x_{2}, x_{1}, x_{4}\right\}$, implies that $T_{1}$ contains $e$ or $x_{4}$. If $e \in T_{1}$, then $\left|F_{1}\right| \geqslant 4$, as $e$ is not in $E(N)$. In this case, $e$ is in a triad that is contained in $F_{1}$, which is a contradiction, as $M^{\prime} \backslash e$ is 3-connected. Therefore $T_{1}$ contains $x_{4}$. Recall that $\left\{e, x_{4}, z\right\}$ is a triangle and $\left\{x_{4}, z, z^{\prime}\right\}$ is a triad of $M_{0}$, and hence of $M^{\prime}$, for some element $z^{\prime}$. Assume $T_{1}$ contains $z$. This implies that $\left\{e, x_{1}, x_{4}, z\right\}$ is a $U_{2,4}$-restriction of $M^{\prime}$ that meets the triad $\left\{x_{4}, z, z^{\prime}\right\}$, which is impossible. Therefore $T_{1}$ contains $z^{\prime}$. By swapping labels on $x_{1}$ and $x_{3}$ in $M^{\prime}$, we see that $\left\{x_{3}, x_{4}, z^{\prime}\right\}$ is a triangle of $M_{0}$. Let $M_{1}$ be the matroid obtained from $M_{0}$ by swapping labels on $x_{2}$ and 
$x_{4}$. As $\left\{x_{2}, x_{4}\right\}$ is a series pair in $M_{0} \backslash e \backslash x_{3}$, it follows that $M_{1} \backslash e \backslash x_{3} / x_{2}=M_{0} \backslash e \backslash x_{3} / x_{2}$, so $M_{1} \backslash e \backslash x_{3} / x_{2}$ has $N$ as a minor. Now $\left\{x_{3}, x_{2}, z^{\prime}\right\}$ is a triangle of $M_{1}$, so $\left\{x_{3}, z^{\prime}\right\}$ is a parallel pair in $M_{1} / x_{2}$. We let $M_{2}$ be the matroid obtained from $M_{1}$ by swapping labels on $x_{3}$ and $z^{\prime}$. Then $M_{2} / x_{2} \backslash x_{3} \backslash e=M_{1} / x_{2} \backslash x_{3} \backslash e$, and hence $M_{2} / x_{2} \backslash x_{3} \backslash e$ has $N$ as a minor. However, $\left\{e, x_{2}, z\right\}$ is a triangle in $M_{2} \backslash x_{3}$, and $\left\{x_{2}, z\right\}$ is a series pair. This contradiction to Proposition 6.2 competes the analysis of Case 1.

Case 2. Statement (ii) holds, so $M^{\prime}$ is obtained from $M_{0}$ by swapping the labels on $x_{2}$ and $x_{4}$. Let $\mathcal{F}^{\prime}$ be a covering family of $M^{\prime}$, and let $F_{3} \in \mathcal{F}^{\prime}$ be the fan that contains $x_{3}$. Note that $x_{3}$ is a terminal spoke element of $F_{3}$ as $M^{\prime} \backslash x_{3}$ is 3 -connected. Let $T_{3}$ be the triangle in $F_{3}$ that contains $x_{3}$. Orthogonality with the cocircuit $\left\{e, x_{2}, x_{3}, x_{4}\right\}$ shows that $e, x_{2}$, or $x_{4}$ is in $T_{3}$. Since $M^{\prime} \backslash e$ is 3 -connected, and $T_{3}-x_{3}$ is contained in a triad, $e$ is not in $T_{3}$. Assume $x_{2}$ is in $T_{3}$. Since $\left\{x_{2}, z, z^{\prime}\right\}$ is a triad of $M^{\prime}$, either $z$ or $z^{\prime}$ is in $T_{3}$. In the former case, $\left\{e, x_{2}, x_{3}, z\right\}$ is a $U_{2,4}$-restriction that intersects a triad, which is a contradiction. Hence $T_{3}=\left\{x_{2}, x_{3}, z^{\prime}\right\}$. As $M^{\prime} / x_{2}$ contains the parallel pair $\left\{x_{3}, z^{\prime}\right\}$ and $N$ as a minor, we can swap labels on $x_{3}$ and $z^{\prime}$ in $M^{\prime}$ and then delete $x_{3}$. The resulting matroid contains $N$ as a minor, a triangle $\left\{e, x_{2}, z\right\}$, and a series pair $\left\{x_{2}, z\right\}$. This contradicts Proposition 6.2, so we deduce that $x_{4}$ is in $T_{3}$. Now $T_{3}=\left\{x_{1}, x_{4}, x_{3}\right\}$, for otherwise $T_{3} \cup x_{1}$ is a $U_{2,4}$-restriction of $M^{\prime} \backslash e$ that intersects a triad. Let $F_{3}=\left(f_{1}, \ldots, f_{s}\right)$. By reversing, we can assume that $f_{s}=x_{3}$.

Proposition 5.6 implies that $\left\{x_{1}, x_{4}, x_{3}\right\}=\left\{f_{s-2}, f_{s-1}, f_{s}\right\}$. Assume that $x_{4} \neq f_{s-1}$. If $s \geqslant 5$, then we let $F_{3}^{\prime}$ be the fan of $M_{0}$ obtained from $F_{3}$ by relabeling $x_{4}$ with $x_{2}$. As $N$ is a minor of $M_{0} / x_{2} \backslash x_{3}$, and $\left\{x_{1}, x_{2}, x_{3}\right\} \subseteq F_{3}^{\prime}$, Proposition 6.7 implies $x_{2}$ and $x_{3}$ are consecutive in $F_{3}^{\prime}$, which implies $x_{4}$ and $x_{3}$ are consecutive in $F_{3}$, contrary to assumption. Therefore $s=4$, and $F_{3}=\left(f_{1}, x_{4}, x_{1}, x_{3}\right)$. Thus $\left(f_{1}, x_{4}, x_{1}\right)$ or its reversal is in $\mathcal{F}_{N}$. Note that $\left\{f_{1}, x_{1}, x_{4}\right\}$ is a triad of $M^{\prime}$, so $\left\{f_{1}, x_{1}, x_{2}\right\}$ is a triad of $M_{0}$, and $\left(f_{1}, x_{1}, x_{2}, x_{3}, x_{4}\right)$ is a fan in $M_{0} \backslash e$. As $M_{0} \backslash e$ is a fan-extension of $N$, it contains a fan that has $\left(f_{1}, x_{4}, x_{1}\right)$ as a subsequence. This fan intersects $\left(f_{1}, x_{1}, x_{2}, x_{3}, x_{4}\right)$ in at least three elements, so it contains $\left\{f_{1}, x_{1}, x_{2}, x_{3}, x_{4}\right\}$, by Lemma 5.8, and therefore contains $\left(f_{1}, x_{1}, x_{2}, x_{3}, x_{4}\right)$ as a contiguous subsequence, by three applications of Proposition 5.6 and its dual. Hence $\left(f_{1}, x_{4}, x_{1}\right)$ is not a subsequence after all. From this contradiction we see that $x_{4}=f_{s-1}$ and $\left(f_{s-2}, f_{s-1}, f_{s}\right)=\left(x_{1}, x_{4}, x_{3}\right)$.

Let $F_{N}$ be the fan in $\mathcal{F}_{N}$ that is consistent with $F_{3}$. Let $\mathcal{F}$ be the family of fans in $M_{0}$ that is induced from $\mathcal{F}^{\prime}$ by swapping the labels on $x_{2}$ and $x_{4}$. As the fans in $\mathcal{F}^{\prime}$ contains all elements in $E\left(M^{\prime}\right)-E(N)$, it follows that the fans in $\mathcal{F}$ contain all elements in $E\left(M_{0}\right)-E(N)$, except possibly for $x_{2}$. However, $x_{4}$ is in $F_{3}$, so $x_{2}$ is in a fan of $\mathcal{F}$. Therefore the fans in $\mathcal{F}$ contain every element in $E\left(M_{0}\right)-E(N)$. As $x_{2}$ does not belong to a fan of $\mathcal{F}_{N}$, the only way $\mathcal{F}$ can fail to be a covering family of $M_{0}$ is if $x_{4}$ is in $F_{N}$. We deduce this is the case. Then $x_{4}$ is a terminal element of $F_{N}$, because $\left(f_{s-2}, f_{s-1}, f_{s}\right)=\left(x_{1}, x_{4}, x_{3}\right)$ and $x_{3} \notin E(N)$.

We claim that $\left\{e, x_{2}, x_{4}\right\}$ is the unique triad of $M_{0} \backslash x_{3}$ that contains $e$. Suppose $T^{*}$ is another such triad. If $x_{2}$ is in $T^{*}$, then $T^{*} \cup x_{4}$ is a $U_{2,4}$-corestriction of $M_{0} \backslash x_{3}$, and $\left\{e, x_{4}, z\right\}$ is a triangle that intersects it. This is a contradiction, so $x_{2} \notin T^{*}$. This means that $T^{*}-e$ is a series pair of $M_{0} \backslash e / x_{2} \backslash x_{3}$, contradicting 6.15.1, and proving the claim. 
As $M_{0} \backslash x_{3}$ is 3 -connected, it contains a covering family.

6.15.4. There exists a covering family of $M_{0} \backslash x_{3}$ containing a fan that contains e and $x_{2}$.

Proof. Let $\mathcal{G}$ be an arbitrary covering family of $M_{0} \backslash x_{3}$, and let $G=\left(g_{1}, \ldots, g_{t}\right)$ be the fan in $\mathcal{G}$ that contains $e$. If $e$ is in a triad that is contained in $G$, then we are done, as this triad must be $\left\{e, x_{2}, x_{4}\right\}$, by the earlier claim. Therefore we assume that $e=g_{1}$ is a terminal spoke element of $G$, and $x_{2}$ is not in $G$. Orthogonality between $\left\{e, x_{2}, x_{4}\right\}$ and $\left\{g_{1}, g_{2}, g_{3}\right\}$ shows that $x_{4} \in\left\{g_{2}, g_{3}\right\}$. If $x_{4}=g_{3}$, then $t=4$, for otherwise we contradict orthogonality between $\left\{e, x_{2}, x_{4}\right\}$ and $\left\{g_{3}, g_{4}, g_{5}\right\}$. In this case $\left(g_{2}, g_{3}, g_{4}\right)=\left(g_{2}, x_{4}, g_{4}\right)$ or its reversal is in $\mathcal{F}_{N}$. Recall $F_{N}$ is the fan in $\mathcal{F}_{N}$ consistent with $F_{3}$, and $x_{4}$ is in $F_{N}$. Hence $F_{N}$ is $\left(g_{2}, x_{4}, g_{4}\right)$ or its reversal, and this is a contradiction, as we concluded $x_{4}$ is a terminal element of $F_{N}$. Therefore $x_{4}=g_{2}$, and $G+x_{2}=\left(x_{2}, g_{1}, \ldots, g_{t}\right)$ is a fan of $M_{0} \backslash x_{3}$. Let $G^{\prime}$ be the fan in $\mathcal{G}$ that contains $x_{2}$. Then $x_{2}$ is a terminal rim element of $G^{\prime}$, by orthogonality with $\left\{e, x_{2}, x_{4}\right\}$. Now $\left(\mathcal{G}-\left\{G, G^{\prime}\right\}\right) \cup\left\{G+x_{2}, G^{\prime}-x_{2}\right\}$ is the desired covering family of $M_{0} \backslash x_{3}$.

Next we claim $M_{0} \backslash x_{3} / x_{2}$ is 3 -connected. Note that $\left(f_{s-3}, f_{s-2}, f_{s-1}, f_{s}\right)=$ $\left(f_{s-3}, x_{1}, x_{4}, x_{3}\right)$ is a fan of $M^{\prime}$, so $\left(f_{s-3}, x_{1}, x_{2}, x_{3}\right)$ is a fan in $M_{0}$. Now Proposition 6.4 tells us that $M_{0} \backslash x_{3} / x_{2}$ is 3 -connected, as desired.

Let $\mathcal{G}$ be a covering family of $M_{0} \backslash x_{3}$, and let $G=\left(g_{1}, \ldots, g_{t}\right)$ be a fan in $\mathcal{G}$ that contains $e$ and $x_{2}$. Then $t \geqslant 5$. As $M_{0} \backslash x_{3} / x_{2}$ is 3 -connected, $x_{2}$ is a terminal rim element of $G$. We can assume $x_{2}=g_{1}$. The only triad of $M_{0} \backslash x_{3}$ that contains $e$ is $\left\{e, x_{2}, x_{4}\right\}$, so if $x_{4}$ is not in $G$ then $e$ is contained in no triad in $G$, implying $e=g_{t}$ is a spoke element. But then $\left\{e, x_{2}, x_{4}\right\}$ violates orthogonality with $\left\{g_{t-2}, g_{t-1}, g_{t}\right\}$. Hence $G$ contains $e, x_{2}$, and $x_{4}$. The dual of Proposition 5.6 implies $\left\{e, x_{2}, x_{4}\right\}=\left\{g_{1}, g_{2}, g_{3}\right\}$. As $t \geqslant 5$, and $e$ is not contained in the triad $\left\{g_{3}, g_{4}, g_{5}\right\}$, we see that $\left(g_{1}, g_{2}, g_{3}\right)=\left(x_{2}, e, x_{4}\right)$.

Since $G$ contains $x_{4}$ it follows that $F_{N}$ is consistent with $G$. Now consider the fans $G$ and $\left(f_{1}, \ldots, f_{s-2}, x_{2}\right)$ in $M_{0} \backslash x_{3}$. They both contain $x_{2}$, and the elements of $F_{N}-x_{4}$. Therefore they intersect in at least three elements, so we can apply Lemma 5.8. This shows that the elements common to both fans form contiguous subsequences in both. This implies $\left\{g_{1}, g_{2}, g_{3}\right\}=\left\{x_{2}, e, x_{4}\right\}$ is a triad contained in $\left(f_{1}, \ldots, f_{s-2}, x_{2}\right)$. Therefore Proposition 5.6 implies $\left\{x_{2}, e, x_{4}\right\}=\left\{x_{2}, f_{s-2}, f_{s-3}\right\}=\left\{x_{2}, x_{1}, f_{s-3}\right\}$. This is impossible as $e, x_{1}$, and $x_{4}$ are distinct elements. We have a contradiction that completes the proof of Lemma 6.15.

If $M_{0} \backslash e$ is 3-connected with $N$ as a minor, where $M_{0}$ is isomorphic to $M$ and is not a fan-extension, then $M_{0} \backslash e$ must be a fan-extension, by the minimality of $M$. This implies $M_{0} \backslash e$ has a covering family. In the next result, we examine this covering family, and discover that the elements in $E\left(M_{0} \backslash e\right)-E(N)$ are concentrated at the ends of fans.

Lemma 6.16. Let $M_{0}$ be isomorphic to $M$. Assume that $N$ is a minor of $M_{0}$, but that $M_{0}$ is not a fan-extension of $N$. Assume also that $M_{0} \backslash e$ is 3-connected and has $N$ as a minor. Let $\mathcal{F}_{e}$ be a covering family of $M_{0} \backslash e$, and let $\left(e_{1}, \ldots, e_{m}\right)$ be a fan in $\mathcal{F}_{e}$. If $2<i<m-1$, then $e_{i}$ is in $E(N)$. 
Proof. Assume that the lemma fails, so that $\left(e_{1}^{\prime}, \ldots, e_{m}^{\prime}\right)$ is a fan in a covering family of $M_{0} \backslash e$ and $e_{j}^{\prime}$ is in $E\left(M_{0} \backslash e\right)-E(N)$, for some $j \in\{3, \ldots, m-2\}$. Note that $m \geqslant 5$.

We claim that either $e_{j-1}^{\prime}$ or $e_{j+1}^{\prime}$ is in $E\left(M_{0} \backslash e\right)-E(N)$. Assume for a contradiction that $\left\{e_{j-1}^{\prime}, e_{j+1}^{\prime}\right\} \subseteq E(N)$. If $e_{j}^{\prime}$ is a rim element in $\left(e_{1}^{\prime}, \ldots, e_{m}^{\prime}\right)$ then let $M^{\prime}$ be $M_{0} \backslash e$ and let $N^{\prime}$ be $N$. Otherwise let $M^{\prime}$ be $\left(M_{0} \backslash e\right)^{*}$ and let $N^{\prime}$ be $N^{*}$. Thus $e_{j}^{\prime}$ is a rim element in the fan $\left(e_{1}^{\prime}, \ldots, e_{m}^{\prime}\right)$ in $M^{\prime}$. Now $N^{\prime}$ is a minor of $M^{\prime} \backslash e_{j}^{\prime}$, for if $N^{\prime}$ is a minor of $M^{\prime} / e_{j}^{\prime}$, then $N^{\prime}$ contains the parallel pair $\left\{e_{j-1}^{\prime}, e_{j+1}^{\prime}\right\}$. Therefore $m=5$ and $j=3$, because otherwise $\left\{e_{j-3}^{\prime}, e_{j-2}^{\prime}, e_{j-1}^{\prime}\right\}$ or $\left\{e_{j+1}^{\prime}, e_{j+2}^{\prime}, e_{j+3}^{\prime}\right\}$ is a codependent triangle in $M^{\prime} \backslash e_{j}^{\prime}$. From $m=5$ and $j=3$, it follows that $\left\{e_{1}^{\prime}, e_{2}^{\prime}, e_{4}^{\prime}, e_{5}^{\prime}\right\}$ is a union of series pairs in $M^{\prime} \backslash e_{j}^{\prime}$, and contains at least three elements of $E\left(N^{\prime}\right)$. This leads to a contradiction to the 3-connectivity of $N^{\prime}$. Therefore $e_{j-1}^{\prime}$ or $e_{j+1}^{\prime}$ is in $E\left(M_{0} \backslash e\right)-E(N)$, as claimed.

By reversing $\left(e_{1}^{\prime}, \ldots, e_{m}^{\prime}\right)$ as necessary, we can assume that $e_{i}^{\prime}$ and $e_{i+1}^{\prime}$ are in $E\left(M_{0} \backslash e\right)-$ $E(N)$, where $1<i<m$, and $e_{i}^{\prime}$ is a rim element. Proposition 6.5 tells us that $N$ is a minor of $M_{0} \backslash e / e_{i}^{\prime} \backslash e_{i+1}^{\prime}$.

By applying Lemma 6.15 to the fan $\left(e_{i-1}^{\prime}, e_{i}^{\prime}, e_{i+1}^{\prime}, e_{i+2}^{\prime}\right)$, we see that we can swap labels on $e_{i-1}^{\prime}$ and $e_{i+1}^{\prime}$ without any loss of generality. If $i-1>1$, we would then be able to apply Lemma 6.15 to the fan $\left(e_{i-2}^{\prime}, e_{i+1}^{\prime}, e_{i}^{\prime}, e_{i-1}^{\prime}\right)$, and swap labels on $e_{i-2}^{\prime}$ and $e_{i}^{\prime}$. Deleting $e$ from the resulting matroid reveals the following fan:

$$
\left(e_{1}, \ldots, e_{i-3}^{\prime}, e_{i}^{\prime}, e_{i+1}^{\prime}, e_{i-2}^{\prime}, e_{i-1}^{\prime}, e_{i+2}^{\prime}, \ldots, e_{m}\right) .
$$

In fact, by starting from $\left(e_{1}^{\prime}, \ldots, e_{i}^{\prime}, e_{i+1}^{\prime}, \ldots, e_{m}^{\prime}\right)$, and repeatedly applying Lemma 6.15, it is possible for us to assume that any sequence of the form

$$
\begin{aligned}
\left(e_{1}^{\prime}, \ldots, e_{j}^{\prime}, x, y, e_{j+1}^{\prime}, \ldots, e_{i-1}^{\prime}, e_{i+2}^{\prime}, \ldots, e_{m}^{\prime}\right) \quad & \text { or } \\
& \left(e_{1}^{\prime}, \ldots, e_{i-1}^{\prime}, e_{i+2}^{\prime}, \ldots, e_{j}^{\prime}, x, y, e_{j+1}^{\prime}, \ldots, e_{m}^{\prime}\right)
\end{aligned}
$$

is a fan of $M_{0} \backslash e$. If we have applied Lemma 6.15 an even number of times in this process, then $(x, y)$ is $\left(e_{i}^{\prime}, e_{i+1}^{\prime}\right)$, otherwise it is $\left(e_{i+1}^{\prime}, e_{i}^{\prime}\right)$. Note that this collection of sequences includes $\left(x, y, e_{1}^{\prime}, \ldots, e_{i-1}^{\prime}, e_{i+2}^{\prime}, \ldots, e_{m}^{\prime}\right)$ and $\left(e_{1}^{\prime}, \ldots, e_{i-1}^{\prime}, e_{i+2}^{\prime}, \ldots, e_{m}^{\prime}, x, y\right)$. We remark that if $i$ had been equal to 1 or $m-1$, then Lemma 6.15 would not have been applicable. This is why it was necessary to show that $i$ lies between 2 and $m-1$.

Since there is a fan of $\mathcal{F}_{N}$ that is consistent with $\left(e_{1}^{\prime}, \ldots, e_{m}^{\prime}\right)$, by repeatedly applying Lemma 6.15, we can assume that we have the following situation: $\left(e_{1}, \ldots, e_{n+2}\right)$ is a contiguous subsequence of $\left(e_{1}^{\prime}, \ldots, e_{m}^{\prime}\right)$, and is therefore a fan of $M_{0} \backslash e$. Some fan $F \in \mathcal{F}_{N}$ is consistent with $\left(e_{1}, \ldots, e_{n}\right)$ and contains $e_{1}$ and $e_{n}$. Also, $e_{n+1}$ and $e_{n+2}$ are not in $E(N)$. Let $\{x, y\}=\left\{e_{n+1}, e_{n+2}\right\}$, where $x$ is a rim element of $\left(e_{1}, \ldots, e_{n+2}\right)$. Then $N$ is a minor of $M_{0} \backslash e / x \backslash y$ by Proposition 6.5.

6.16.1. Let $e_{i}$ be a rim element of $\left(e_{1}, \ldots, e_{n+2}\right)$, where $1 \leqslant i \leqslant n$. Then $\left\{e_{i}, e_{i+1}, e_{i+2}\right\}$ is not a triad in $M_{0}$.

Proof. Assume this is false. By starting from $\left(e_{1}^{\prime}, \ldots, e_{m}^{\prime}\right)$, and using Lemma 6.15 to shift $x$ and $y$, we can, without losing any generality, assume that $\left(e_{1}, \ldots, e_{i-1}, x, y, e_{i}, \ldots, e_{n}\right)$ is a fan of $M_{0} \backslash e$. Here $x$ is a rim element and $\left\{x, y, e_{i}\right\}$ is a triad of $M_{0}$, while $N$ is a minor of 
$M_{0} \backslash e / x \backslash y$. By Proposition 6.4, $M_{0} \backslash e / x \backslash y$ is 3-connected. If $M_{0} / x \backslash y$ is not 3-connected, there is a triangle of $M_{0}$ that contains $x$ and $e$ but not $y$. Orthogonality with $\left\{x, y, e_{i}\right\}$ shows that $\left\{e, x, e_{i}\right\}$ is a triangle of $M_{0}$, so $\left\{e, x, e_{i}\right\}$ is a codependent triangle in $M_{0} \backslash y$. This contradiction shows that $M_{0} / x \backslash y$ is 3 -connected, and is therefore a fan-extension of $N$.

Let $F^{\prime}$ be the fan in a covering family of $M_{0} / x \backslash y$ such that $F$ is consistent with $F^{\prime}$. Now $\left(e_{1}, \ldots, e_{n}\right)$ is a fan of $M_{0} / x \backslash y \backslash e$, by Proposition 5.2 , and $F$ is consistent with $\left(e_{1}, \ldots, e_{n}\right)$ and contains $e_{1}$ and $e_{n}$. Note that if $e$ is in $F^{\prime}$, then it is a terminal spoke element, as $M_{0} / x \backslash y \backslash e$ is 3 -connected. Hence $F^{\prime}-e$ is a fan of $M_{0} / x \backslash y \backslash e$. Because $F^{\prime}-e$ and $\left(e_{1}, \ldots, e_{n}\right)$ have at least three elements in common, and these elements include $e_{1}$ and $e_{n}$, it follows from Lemma 5.8 that the elements in $\left\{e_{1}, \ldots, e_{n}\right\}$ form a contiguous subsequence of $F^{\prime}-e$.

Assume that the elements $\left\{e_{1}, \ldots, e_{n}\right\}$ do not appear in the order $\left(e_{1}, \ldots, e_{n}\right)$ in $F^{\prime}-e$. Then it follows easily from Proposition 5.6 that $n<5$. Note that $F$ is consistent with $\left(e_{1}, \ldots, e_{n}\right)$ and with $F^{\prime}$, so the elements of $F$ appear in $F^{\prime}$ in the same order as they do in $\left(e_{1}, \ldots, e_{n}\right)$. Therefore our assumption means that $F$ is not equal to $\left(e_{1}, \ldots, e_{n}\right)$ or its reversal, although it contains $e_{1}$ and $e_{n}$. If $n=3$, then $F$ is equal to $\left(e_{1}, e_{2}, e_{3}\right)$ or its reversal, contrary to our conclusion. Therefore $n=4$, and $F$ is either $\left(e_{1}, e_{2}, e_{4}\right)$ or $\left(e_{1}, e_{3}, e_{4}\right)$, or one of their reversals. Now $\left\{e_{1}, e_{2}, e_{3}, e_{4}\right\}$ is contained in $E(N)$, or else $N$ contains a series or parallel pair. Therefore $\left(e_{1}, e_{2}, e_{3}, e_{4}\right)$ is a fan of $N$. As $\left(e_{1}, e_{2}, e_{4}\right)$ or $\left(e_{1}, e_{3}, e_{4}\right)$ is also a fan of $N$, we have contradicted Proposition 5.6. Thus $\left(e_{1}, \ldots, e_{n}\right)$ is a contiguous subsequence of $F^{\prime}-e$, and hence of $F^{\prime}$.

Let $F^{\prime}$ be $\left(f_{1}, \ldots, f_{j-1}, e_{1}, \ldots, e_{n}, f_{j}, \ldots, f_{p}\right)$, so this sequence is a fan in $M_{0} / x \backslash y$. We will show that

$$
\left(f_{1}, \ldots, f_{j-1}, e_{1}, \ldots, e_{i-1}, x, y, e_{i}, \ldots, e_{n}, f_{j}, \ldots, f_{p}\right)
$$

is a fan in $M_{0}$. Let this sequence be labeled $\left(g_{1}, \ldots, g_{k-1}, x, y, g_{k}, \ldots, g_{r}\right)$, so that $\left(g_{1}, \ldots, g_{r}\right)$ is a fan in $M_{0} / x \backslash y$. Note that $i \leqslant n$, so there is at least one element in $\left\{g_{k}, \ldots, g_{r}\right\}$. By assumption, $\left\{x, y, e_{i}\right\}=\left\{x, y, g_{k}\right\}$ is a triad of $M_{0}$.

Assume that $r \geqslant k+1$. If $g_{k+1}=e_{i+1}$, then $\left\{y, e_{i}, e_{i+1}\right\}=\left\{y, g_{k}, g_{k+1}\right\}$ is a triangle of $M_{0}$, since $\left(e_{1}, \ldots, e_{i-1}, x, y, e_{i}, \ldots, e_{n}\right)$ is a fan in $M_{0} \backslash e$. On the other hand, if $g_{k+1}=f_{j}$, then $i=n$, and $\left\{e_{n-1}, e_{n}, f_{j}\right\}=\left\{g_{k-1}, g_{k}, g_{k+1}\right\}$ is a triangle in $M_{0} / x \backslash y$. It cannot be a triangle in $M_{0}$ by orthogonality with the triad $\left\{x, y, g_{k}\right\}$. Therefore $\left\{g_{k-1}, x, g_{k}, g_{k+1}\right\}$ is a circuit in $M_{0}$. Now $\left\{g_{k-1}, x, y\right\}$ is a triangle of $M_{0} \backslash e$, and therefore of $M_{0}$. Strong circuit-exchange between $\left\{g_{k-1}, x, g_{k}, g_{k+1}\right\}$ and $\left\{g_{k-1}, x, y\right\}$ shows that there is a circuit in $\left\{g_{k-1}, y, g_{k}, g_{k+1}\right\}$ that contains $y$. Orthogonality shows that this circuit cannot contain $g_{k-1}$, because $n \geqslant 3$, and $\left\{e_{n-2}, e_{n-1}, x\right\}=\left\{g_{k-2}, g_{k-1}, x\right\}$ is a triad of $M_{0} \backslash e$. Therefore $\left\{y, g_{k}, g_{k+1}\right\}$ is a triangle in $M_{0}$ in any case.

Next we assume that $r \geqslant k+2$. Then $\left\{g_{k}, g_{k+1}, g_{k+2}\right\}$ is a triad in $M_{0} / x \backslash y$. Assume that it is not a triad in $M_{0}$, so that $\left\{y, g_{k}, g_{k+1}, g_{k+2}\right\}$ is a cocircuit. If $n \geqslant i+2$, then $\left\{e_{i}, e_{i+1}, e_{i+2}\right\}=\left\{g_{k}, g_{k+1}, g_{k+2}\right\}$ is a triad in $M_{0} \backslash e$, and $\left\{y, g_{k}, g_{k+1}, g_{k+2}\right\}$ is a union of cocircuits. Therefore $\left\{y, g_{k}, g_{k+1}, g_{k+2}\right\}$ is a $U_{2,4}$-corestriction of $M_{0} \backslash e$. As $\left\{y, g_{k}, g_{k+1}\right\}$ is a triangle this is a contradiction to Corollary 6.3 , so $n<i+2$, meaning that $i>1$. Now $\left\{e_{i-1}, x, y\right\}=\left\{g_{k-1}, x, y\right\}$ is a triangle of $M_{0} \backslash e$ that violates orthogonality with 
$\left\{y, g_{k}, g_{k+1}, g_{k+2}\right\}$. Hence $\left\{g_{k}, g_{k+1}, g_{k+2}\right\}$ is a triad in $M_{0}$.

Now we consider all the sets comprising three consecutive elements in $\left(g_{k+1}, \ldots, g_{r}\right)$. If such a set is a triangle in $M_{0} / x \backslash y$, then it is a triangle in $M_{0}$, by orthogonality with the triad $\left\{x, y, g_{k}\right\}$. If such a set is a triad in $M_{0} / x \backslash y$, then it is a triad in $M_{0}$ by orthogonality with the triangle $\left\{y, g_{k}, g_{k+1}\right\}$. We have shown that $\left(x, y, g_{k}, \ldots, g_{r}\right)$ is a fan in $M_{0}$.

Assume that $k>1$. If $g_{k-1}=e_{i-1}$, then $\left\{g_{k-1}, x, y\right\}$ is a triangle in $M_{0}$, since $\left(e_{1}, \ldots, e_{i-1}, x, y, e_{i}, \ldots, e_{n}\right)$ is a fan of $M_{0} \backslash e$. Therefore we assume that $g_{k-1}=f_{j-1}$. This means that $i=1$, which implies that $\left\{g_{k}, \ldots, g_{r}\right\}$ contains at least three elements. Note $\left\{f_{j-1}, e_{1}, e_{2}\right\}=\left\{g_{k-1}, g_{k}, g_{k+1}\right\}$ is a triangle in $M_{0} / x \backslash y$, but not in $M_{0}$, by orthogonality with the triad $\left\{x, y, g_{k}\right\}$. Therefore $\left\{g_{k-1}, x, g_{k}, g_{k+1}\right\}$ is a circuit in $M_{0}$. We perform strong circuit-exchange on $\left\{g_{k-1}, x, g_{k}, g_{k+1}\right\}$ and the triangle $\left\{y, g_{k}, g_{k+1}\right\}$ to obtain a circuit of $M_{0}$ contained in $\left\{g_{k-1}, x, y, g_{k+1}\right\}$ that contains $y$. Orthogonality with the triad $\left\{g_{k}, g_{k+1}, g_{k+2}\right\}$ shows that this circuit cannot contain $g_{k+1}$. Therefore $\left\{g_{k-1}, x, y\right\}$ is a triangle of $M_{0}$ in any case.

Next we assume that $k>2$. We wish to show that $\left\{g_{k-2}, g_{k-1}, x\right\}$ is a triad in $M_{0}$. Now $\left\{g_{k-2}, g_{k-1}, g_{k}\right\}$ is a triad in $M_{0} / x \backslash y$. It is not a triad in $M_{0}$, by orthogonality with the triangle $\left\{g_{k-1}, x, y\right\}$, so $\left\{g_{k-2}, g_{k-1}, y, g_{k}\right\}$ is a cocircuit of $M_{0}$. By strong cocircuitexchange with $\left\{x, y, g_{k}\right\}$, we find a cocircuit, $C^{*}$, of $M_{0}$ contained in $\left\{g_{k-2}, g_{k-1}, x, g_{k}\right\}$ that contains $x$. If $C^{*}$ does not contain $g_{k}$, then $\left\{g_{k-2}, g_{k-1}, x\right\}$ is a triad, as desired. Therefore we assume that $g_{k} \in C^{*}$. Orthogonality with the triangle $\left\{g_{k-1}, x, y\right\}$ shows that $g_{k-1} \in C^{*}$. Because $\left\{x, y, g_{k}\right\}$ is a triad of $M_{0}$, it follows that if $C^{*}=\left\{g_{k-1}, x, g_{k}\right\}$, then $C^{*} \cup y$ is a $U_{2,4^{-}}$-corestriction of $M_{0}$. As $\left\{g_{k-1}, x, y\right\}$ is a triangle, this contradicts Corollary 6.3. Hence $C^{*}=\left\{g_{k-2}, g_{k-1}, x, g_{k}\right\}$. It follows that $r=k$, for otherwise $C^{*}$ and $\left\{y, g_{k}, g_{k+1}\right\}$ violate orthogonality. From $r=k$, we deduce that $i=n$, and as $n \geqslant 3$, it follows that $\left(g_{k-2}, g_{k-1}\right)=\left(e_{n-2}, e_{n-1}\right)$, so $\left\{g_{k-2}, g_{k-1}, x\right\}$ is a triad in $M_{0} \backslash e$. Now $C^{*}$ is a union of cocircuits in $M_{0} \backslash e$ that contains the triad $\left\{g_{k-2}, g_{k-1}, x\right\}$, so $C^{*}$ is a $U_{2,4}$-corestriction in $M_{0} \backslash e$. Since $\left\{g_{k-1}, x, y\right\}$ is a triangle, this is impossible. Therefore $\left\{g_{k-2}, g_{k-1}, x\right\}$ is a triad in $M_{0}$, as we claimed.

Finally, we observe that any set of three consecutive elements from $\left(g_{1}, \ldots, g_{k-1}\right)$ that is a triangle in $M_{0} / x \backslash y$ is a triangle in $M_{0}$, since $\left\{x, y, g_{k}\right\}$ is a triad. Any such set that is a triad in $M_{0} / x \backslash y$ is a triad in $M_{0}$, as $\left\{g_{k-1}, x, y\right\}$ is a triangle.

We have shown that

$$
\left(g_{1}, \ldots, g_{k-1}, x, y, g_{k}, \ldots, g_{r}\right)=\left(f_{1}, \ldots, f_{j-1}, e_{1}, \ldots, e_{i-1}, x, y, e_{i}, \ldots, e_{n}, f_{j}, \ldots, f_{p}\right)
$$

is a fan of $M_{0}$. Now $M_{0} / x \backslash y$ has a covering family containing $F^{\prime}=$ $\left(f_{1}, \ldots, f_{j-1}, e_{1}, \ldots, e_{n}, f_{j}, \ldots, f_{p}\right)$, and $M_{0}$ is obtained from $M_{0} / x \backslash y$ by a fan-lengthening move on this fan. Hence $M_{0}$ is a fan-extension of $N$. This contradiction completes the proof of 6.16.1.

6.16.2. If $e_{i}$ is a rim element of $\left(e_{1}, \ldots, e_{n+2}\right)$, and there is a triad of $M_{0} \backslash e$ contained in $\left\{e_{1}, \ldots, e_{n+2}\right\}$ that does not contain $e_{i}$, then there is a triangle of $M_{0}$ containing $\left\{e, e_{i}\right\}$.

Proof. Assume that the claim fails. We will start from $\left(e_{1}^{\prime}, \ldots, e_{m}^{\prime}\right)$, and then use Lemma 6.15 to shift $x$ and $y$ appropriately. By then possibly reversing, we can assume 
that $\left(e_{1}, \ldots, e_{i-1}, x, y, e_{i}, \ldots, e_{n}\right)$ is a fan of $M_{0} \backslash e$, where $x$ is a rim element and $M_{0} \backslash e / x \backslash y$ has $N$ as a minor; moreover, we assume there is no triangle of $M_{0}$ containing $\{e, x\}$, and $\left\{e_{j}, e_{j+1}, e_{j+2}\right\}$ is a triad of $M_{0} \backslash e$, for some $j \in\{i, \ldots, n-2\}$. Proposition 6.4 implies that $M_{0} \backslash e / x \backslash y$ is 3-connected. Because there is no triangle containing $e$ and $x$ in $M_{0}$, it follows that $M_{0} / x \backslash y$ is 3-connected, and hence a fan-extension of $N$. Let $F^{\prime}$ be the fan in a covering family of $M_{0} / x \backslash y$ such that $F$ is consistent with $F^{\prime}$. If $F^{\prime}$ contains $e$, then $e$ is a terminal element because $M_{0} / x \backslash y \backslash e$ is 3-connected, so $F^{\prime}-e$ is a fan of $M_{0} / x \backslash y \backslash e$. Now $F$ is also consistent with $\left(e_{1}, \ldots, e_{n}\right)$, which is a fan of $M_{0} / x \backslash y \backslash e$. Since $\left(e_{1}, \ldots, e_{n}\right)$ and $F^{\prime}-e$ have at least three elements in common, including $e_{1}$ and $e_{n}$, it follows from Lemma 5.8 that the elements in $\left\{e_{1}, \ldots, e_{n}\right\}$ form a contiguous subsequence of $F^{\prime}-e$. As $\left\{e_{j}, e_{j+1}, e_{j+2}\right\}$ is a triad in $M_{0} / x \backslash y \backslash e$, it is a set of three consecutive elements in $F^{\prime}$ by the dual of Proposition 5.6. It cannot be a triangle in $M_{0} / x \backslash y$, for then it would be a triad and a triangle in $M_{0} / x \backslash y \backslash e$. Therefore $\left\{e_{j}, e_{j+1}, e_{j+2}\right\}$ is a triad in $M_{0} / x \backslash y$, but not in $M_{0}$ by 6.16.1. This shows that $y$ is in the coclosure of $\left\{e_{i}, \ldots, e_{n}\right\}$ in $M_{0}$, and hence in $M_{0} \backslash e$. It is in the closure of the same set because $\left\{y, e_{i}, e_{i+1}\right\}$ is a triangle. Thus $\left\{y, e_{i}, \ldots, e_{n}\right\}$ is 2 -separating in $M_{0} \backslash e$, so the complement of $\left\{y, e_{i}, \ldots, e_{n}\right\}$ contains at most one element. Proposition 5.4 implies $M_{0} \backslash e$ is a wheel or a whirl. This contradiction completes the proof of 6.16.2.

6.16.3. $n \leqslant 5$, and if $n=5$, then $e_{1}$ is a spoke element of $\left(e_{1}, \ldots, e_{n+2}\right)$.

Proof. If this statement is false, then, by starting from $\left(e_{1}^{\prime}, \ldots, e_{m}^{\prime}\right)$, and applying Lemma 6.15 to shift $x$ and $y$ as necessary, we can let $\left(x_{1}, \ldots, x_{5}, y, x\right)$ be either $\left(e_{1}, \ldots, e_{5}, y, x\right)$ or $\left(e_{2}, \ldots, e_{6}, y, x\right)$, and assume that $\left(x_{1}, \ldots, x_{5}, y, x\right)$ is a fan of $M_{0} \backslash e$, where $N$ is a minor of $M_{0} \backslash e / x \backslash y$, and $x_{1}$ is a rim element of $\left(x_{1}, \ldots, x_{5}, y, x\right)$. Since $\left\{x_{1}, x_{2}, x_{3}\right\},\left\{x_{3}, x_{4}, x_{5}\right\}$, and $\left\{x_{5}, y, x\right\}$ are triads in $M_{0} \backslash e$, we apply 6.16 .1 and 6.16 .2 and see that $\left\{e, x_{1}, x_{2}, x_{3}\right\},\left\{e, x_{3}, x_{4}, x_{5}\right\}$, and $\left\{e, x_{5}, y, x\right\}$ are cocircuits in $M_{0}$, and that $\left\{e, x_{1}\right\},\left\{e, x_{3}\right\},\left\{e, x_{5}\right\}$, and $\{e, x\}$ are all contained in triangles of $M_{0}$. Now it is easy to see, using orthogonality, that $\left\{e, x_{1}, x_{5}\right\}$ and $\left\{e, x_{3}, x\right\}$ are triangles of $M_{0}$. This implies that $x \in \operatorname{cl}_{M_{0} \backslash e}\left(\left\{x_{1}, \ldots, x_{5}, y\right\}\right)$. Since $x$ is also in $\mathrm{cl}_{M_{0} \backslash e}^{*}\left(\left\{x_{1}, \ldots, x_{5}, y\right\}\right)$, we see that $\left\{x_{1}, \ldots, x_{5}, y, x\right\}$ is 2-separating in $M_{0} \backslash e$, and it follows that $M_{0} \backslash e$ is a wheel or a whirl. This contradiction completes the proof.

6.16.4. $n \leqslant 4$.

Proof. If $n>4$, then by 6.16.3, $n=5$, and $e_{1}$ is a spoke element. Therefore $\left(e_{1}, \ldots, e_{5}, x, y\right)$ is a fan of $M_{0} \backslash e$, where $N$ is a minor of $M_{0} \backslash e / x \backslash y$. As $\left\{e_{2}, e_{3}, e_{4}\right\}$ and $\left\{e_{4}, e_{5}, x\right\}$ are triads of $M_{0} \backslash e, 6.16 .1$ and 6.16.2 imply that $\left\{e, e_{2}, e_{3}, e_{4}\right\}$ and $\left\{e, e_{4}, e_{5}, x\right\}$ are cocircuits of $M_{0}$, and $\left\{e, e_{2}\right\}$ and $\{e, x\}$ are contained in triangles of $M_{0}$. Assume $\left\{e, e_{2}, x\right\}$ is not a triangle. Then orthogonality requires that $\left\{e, e_{2}\right\}$ is in a triangle with $e_{4}$ or $e_{5}$, and that $\{e, x\}$ is in a triangle with $e_{3}$ or $e_{4}$. This implies that $x$ is in the closure and the coclosure of $\left\{e_{1}, \ldots, e_{5}\right\}$ in $M_{0} \backslash e$, and we arrive at the contradiction that $M_{0} \backslash e$ is a wheel or a whirl. Therefore $\left\{e, e_{2}, x\right\}$ is a triangle.

Assume $M_{0} \backslash y$ is not 3 -connected. Then there is a triad of $M_{0}$ that contains $y$. By orthogonality with $\left\{e_{5}, x, y\right\},\left\{e_{3}, e_{4}, e_{5}\right\}$, and $\left\{e, e_{2}, x\right\}$, we see that $y$ is in the coclosure 
and closure of $\left\{e_{1}, \ldots, e_{5}, x\right\}$ in $M_{0}$, and also in $M_{0} \backslash e$. This leads to a contradiction as before, so $M_{0} \backslash y$ is 3-connected, and therefore a fan-extension of $N$. This implies there is a fan, $F^{\prime}$, of $M_{0} \backslash y$ such that $F$ is consistent with $F^{\prime}$ and $e_{1}$ and $e_{5}$ are terminal elements of $F^{\prime}$.

Proposition 6.4 implies that $M_{0} \backslash y \backslash e / x$ is 3 -connected. If neither $e$ nor $x$ is an internal element of $F^{\prime}$, then $F^{\prime}-\{e, x\}$ is a fan of $M_{0} \backslash y \backslash e / x$ by the fact that $M_{0} \backslash y \backslash e / x$ is 3-connected. Assume either $e$ or $x$ is an internal element in $F^{\prime}$. By applying Proposition 6.6 to $M^{\prime}=M_{0} \backslash y$, we see that $e$ and $x$ are consecutive in $F^{\prime}$, and $x$ is a rim element. Then Proposition 5.2 implies $F^{\prime}-\{e, x\}$ is a fan of $M_{0} \backslash y \backslash e / x$, so this is true in any case. We note that $\left(e_{1}, \ldots, e_{5}\right)$ is also a fan in $M_{0} \backslash y \backslash e / x$. As $F^{\prime}-\{e, x\}$ and $\left(e_{1}, \ldots, e_{5}\right)$ have at least three elements in common, including $e_{1}$ and $e_{5}$, Lemma 5.8 shows that $\left\{e_{1}, \ldots, e_{5}\right\}$ is contained in $F^{\prime}-\{e, x\}$. Applying Proposition 5.6 three times shows that $\left(e_{1}, \ldots, e_{5}\right)$ is a contiguous subsequence of $F^{\prime}-\{e, x\}$. Assume $\left(e_{2}, e_{3}, e_{4}\right)$ is a contiguous subsequence of $F^{\prime}$. Since $\left\{e_{2}, e_{3}, e_{4}\right\}$ is a triad in $M_{0} \backslash y \backslash e / x$, it cannot be a triangle in $M_{0} \backslash y$. Hence it is a triad in $M_{0} \backslash y$, but not in $M_{0}$. This means $y$ is in the coclosure and the closure of $\left\{e_{1}, \ldots, e_{5}, x\right\}$ in $M_{0} \backslash e$. This leads to a contradiction, so $\left(e_{2}, e_{3}, e_{4}\right)$ is not a contiguous subsequence of $F^{\prime}$. This means that the elements $\left\{e, x, e_{3}\right\}$ are consecutive in $F^{\prime}$, so $\left\{e, x, e_{3}\right\}$ is a triad or a triangle in $M_{0} \backslash y$. The former case is impossible, by orthogonality with $\left\{e_{3}, e_{4}, e_{5}\right\}$. Therefore $\left\{e, x, e_{3}\right\}$ is a triangle of $M_{0} \backslash y$. Since $\left\{e, x, e_{2}\right\}$ is also a triangle, we see that $\left\{e, x, e_{2}, e_{3}\right\}$ is a $U_{2,4}$-restriction of $M_{0} \backslash y$ that is contained in the fan $F^{\prime}$. Therefore it intersects a triad, and this contradiction to Corollary 6.3 shows that $n \leqslant 4$.

\subsection{5. $n=3$.}

Proof. If $n>3$, then by $6.16 .4, n=4$. If $e_{1}$ is a rim element, we let $\left(x_{1}, x_{2}, x_{3}, x_{4}, x, y\right)$ be $\left(e_{1}, \ldots, e_{6}\right)$. Otherwise, we shift $x$ and $y$ using Lemma 6.15 so that $\left(y, x, e_{1}, e_{2}, e_{3}, e_{4}\right)$ is a fan of $M_{0} \backslash e$, and now we let $\left(x_{1}, x_{2}, x_{3}, x_{4}, x, y\right)$ be $\left(e_{4}, e_{3}, e_{2}, e_{1}, x, y\right)$. In either case $\left(x_{1}, x_{2}, x_{3}, x_{4}, x, y\right)$ is a fan of $M_{0} \backslash e$ with $x_{1}$ as a rim element, and $N$ is a minor of $M_{0} / x \backslash y$. Since $\left\{x_{1}, x_{2}, x_{3}\right\}$ and $\left\{x_{3}, x_{4}, x\right\}$ are triads of $M_{0} \backslash e$, we deduce from 6.16.1 and 6.16.2 that $\left\{e, x_{1}, x_{2}, x_{3}\right\}$ and $\left\{e, x_{3}, x_{4}, x\right\}$ are cocircuits of $M_{0}$, and that $\left\{e, x_{1}\right\}$ and $\{e, x\}$ are contained in triangles. If $\left\{e, x_{1}, x\right\}$ is not a triangle, then $x$ is contained in the closure and the coclosure of $\left\{x_{1}, x_{2}, x_{3}, x_{4}\right\}$ in $M_{0} \backslash e$, exactly as in the proof of 6.16.4. Because this leads to a contradiction, $\left\{e, x_{1}, x\right\}$ is a triangle of $M_{0}$.

If $M_{0} \backslash y$ is not 3-connected, then there is a triad of $M_{0}$ containing $y$, and $y$ is in the coclosure and the closure of $\left\{x_{1}, x_{2}, x_{3}, x_{4}, x\right\}$ in $M_{0} \backslash e$, as in the proof of 6.16.4. This gives a contradiction, so $M_{0} \backslash y$ is 3-connected, and hence a fan-extension of $N$. There is a fan, $F^{\prime}$, in $M_{0} \backslash y$ such that $F$ is consistent with $F^{\prime}$, and $x_{1}$ and $x_{4}$ are terminal elements of $F^{\prime}$. If neither $e$ nor $x$ is an internal element of $F^{\prime}$, then $F^{\prime}-\{e, x\}$ is a fan of $M_{0} \backslash y \backslash e / x$. If either $e$ or $x$ is an internal element, then Propositions 5.2 and 6.6 show $F^{\prime}-\{e, x\}$ is a fan of $M_{0} \backslash y \backslash e / x$. As $\left(x_{1}, x_{2}, x_{3}, x_{4}\right)$ is also a fan in $M_{0} \backslash y \backslash e / x$, the elements $\left\{x_{1}, x_{2}, x_{3}, x_{4}\right\}$ form a contiguous subsequence of $F^{\prime}-\{e, x\}$.

Assume $\left(x_{1}, x_{2}, x_{3}, x_{4}\right)$ is not a contiguous subsequence of $F^{\prime}-\{e, x\}$. As $F$ is consistent with $F^{\prime}-\{e, x\}$, it follows that, up to reversing, $F$ is either $\left(x_{1}, x_{2}, x_{4}\right)$ or $\left(x_{1}, x_{3}, x_{4}\right)$. In 
this case $\left\{x_{1}, x_{2}, x_{3}, x_{4}\right\} \subseteq E(N)$, for otherwise $N$ contains a series or parallel pair. But then $\left(x_{1}, x_{2}, x_{3}, x_{4}\right)$ is a fan of $N$, and so is either $\left(x_{1}, x_{2}, x_{4}\right)$ or $\left(x_{1}, x_{3}, x_{4}\right)$, contradicting Proposition 5.6. Therefore $\left(x_{1}, x_{2}, x_{3}, x_{4}\right)$ is a contiguous subsequence of $F^{\prime}-\{e, x\}$. Assume $\left(x_{1}, x_{2}, x_{3}\right)$ is a contiguous subsequence of $F^{\prime}$. Then $\left\{x_{1}, x_{2}, x_{3}\right\}$ is a triad of $M_{0} \backslash y$ but not of $M_{0}$, so $y$ is in the coclosure and closure of $\left\{x_{1}, x_{2}, x_{3}, x_{4}, x\right\}$ in $M_{0} \backslash e$. This leads to a contradiction, so $\left(x_{1}, x_{2}, x_{3}\right)$ is not a contiguous subsequence, and thus $e, x$, and $x_{2}$ are consecutive in $F^{\prime}$. By orthogonality with $\left\{x_{2}, x_{3}, x_{4}\right\},\left\{e, x, x_{2}\right\}$ is not a triad in $M_{0} \backslash y$, so $\left\{e, x, x_{2}\right\}$ is a triangle of $M_{0} \backslash y$. Thus $\left\{e, x_{1}, x_{2}, x\right\}$ is a $U_{2,4}$-restriction of $M_{0} \backslash y$ that intersects a triad contained in $F^{\prime}$. This contradiction completes the proof.

\subsection{6. $e_{1}$ is a spoke element.}

Proof. Assume that $e_{1}$ is a rim element. Then $\left(e_{1}, e_{2}, e_{3}, y, x\right)$ is a fan of $M_{0} \backslash e$, and $F$ is equal to $\left(e_{1}, e_{2}, e_{3}\right)$ or its reversal. As before, we see that $\left\{e, e_{1}, e_{2}, e_{3}\right\}$ and $\left\{e, e_{3}, y, x\right\}$ are cocircuits in $M_{0}$, and $\left\{e, e_{1}, x\right\}$ is a triangle.

Assume $M_{0} \backslash y$ is not 3 -connected, so that $y$ is contained in a triad, $T^{*}$, of $M_{0}$. Note $T^{*}$ is a triad of $M_{0} \backslash e$ but $T^{*} \neq\left\{e_{3}, y, x\right\}$. If $T^{*} \cap\left\{e_{3}, x\right\} \neq \emptyset$, then $T^{*} \cup\left\{e_{3}, x\right\}$ is a $U_{2,4}$-corestriction in $M_{0} \backslash e$, and $\left\{e_{2}, e_{3}, y\right\}$ is a triangle. This contradiction to Corollary 6.3 shows that $T^{*} \cap\left\{e_{3}, x\right\}=\emptyset$. Orthogonality with $\left\{e_{2}, e_{3}, y\right\}$ now shows that $e_{2}$ is in $T^{*}$. Assume that we swap labels on $e_{3}$ and $x$ in $M_{0}$, and then contract $x$. The resulting matroid has $N$ as a minor, as $\left\{e_{3}, x\right\}$ is a series pair in $M_{0} \backslash e \backslash y$. It also has the triad $T^{*}$, which contains the parallel pair $\left\{e_{2}, y\right\}$. This contradiction shows that $M_{0} \backslash y$ is 3-connected, and therefore a fan-extension of $N$.

Let $F^{\prime}$ be a fan of $M_{0} \backslash y$ such that $\left(e_{1}, e_{2}, e_{3}\right)$ is consistent with $F^{\prime}$, and $e_{1}$ and $e_{3}$ are terminal elements. Now $M_{0} \backslash y \backslash e / x$ is 3-connected, and contains the triad $\left\{e_{1}, e_{2}, e_{3}\right\}$. As before, $F^{\prime}-\{e, x\}$ is a fan of $M_{0} \backslash y \backslash e / x$. It follows that $\left(e_{1}, e_{2}, e_{3}\right)$ is a contiguous subsequence of $F^{\prime}-\{e, x\}$. If $\left(e_{1}, e_{2}, e_{3}\right)$ is a contiguous subsequence of $F^{\prime}$, then $\left\{e_{1}, e_{2}, e_{3}\right\}$ is a triad of $M_{0} \backslash y$, since it cannot be a triangle and a triad in $M_{0} \backslash y \backslash e / x$. Therefore $\left\{e_{1}, e_{2}, e_{3}\right\}$ is a triad in $M_{0} \backslash y$ but not in $M_{0}$. This means $\left\{e_{1}, e_{2}, e_{3}, y\right\}$ is 2-separating in $M_{0} \backslash e$, leading to the contradiction that $M_{0} \backslash e$ is a wheel or a whirl. Therefore $\left(e_{1}, e_{2}, e_{3}\right)$ is not a contiguous subsequence of $F^{\prime}$, so $\left\{e, x, e_{2}\right\}$ is a set of consecutive elements in $F^{\prime}$. If $\left\{e, x, e_{2}\right\}$ is a triad in $M_{0} \backslash y$, then $\left\{e, x, e_{2}, y\right\}$ is a cocircuit in $M_{0}$, by orthogonality with $\left\{e_{2}, e_{3}, y\right\}$. Therefore $\left\{e_{2}, y, x\right\}$ and $\left\{e_{3}, y, x\right\}$ are both triads in $M_{0} \backslash e$, so $M_{0} \backslash e$ has a $U_{2,4}$-corestriction that intersects a triangle, a contradiction. This shows that $\left\{e, x, e_{2}\right\}$ is a triangle in $M_{0} \backslash y$, so $\left\{e, x, e_{1}, e_{2}\right\}$ is a $U_{2,4}$-restriction of $M_{0} \backslash y$ that intersects a triad contained in $F^{\prime}$, a contradiction.

Now we know that $e_{1}$ is a spoke element and $\left(e_{1}, e_{2}, e_{3}\right)$ or its reversal is in $\mathcal{F}_{N}$. By shifting $x$ and $y$, we can assume that $\left(e_{1}, e_{2}, y, x, e_{3}\right)$ is a fan in $M_{0} \backslash e$ with $e_{1}$ as a spoke element, and $N$ is a minor of $M_{0} \backslash e / x \backslash y$. It follows from 6.16.1 that $\left\{e, e_{2}, y, x\right\}$ is a cocircuit in $M_{0}$. Assume that $y$ is in a triad, $T^{*}$, of $M_{0}$. Then $T^{*} \neq\left\{e_{2}, y, x\right\}$, and $T^{*}$

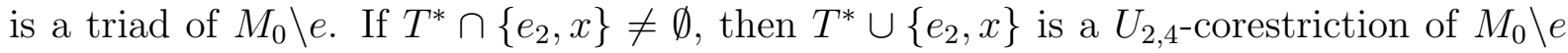
that intersects a triangle, which is impossible. Therefore $T^{*} \cap\left\{e_{2}, x\right\}=\emptyset$. Orthogonality shows that $T^{*}=\left\{e_{1}, y, e_{3}\right\}$. This means $M_{0} / x$ contains a dependent triad, so we have a 
contradiction. We conclude that $y$ is in no triad in $M_{0}$, so $M_{0} \backslash y$ is 3 -connected, and is therefore a fan-extension of $N$.

Let $F^{\prime}$ be a fan of $M_{0} \backslash y$ such that $\left(e_{1}, e_{2}, e_{3}\right)$ is a subsequence of $F^{\prime}$ and $e_{1}$ and $e_{3}$ are terminal elements. As $\left\{e_{1}, e_{2}, e_{3}\right\}$ is a triangle in $M_{0} \backslash y \backslash e / x$, we conclude as before that $F^{\prime}-\{e, x\}=\left(e_{1}, e_{2}, e_{3}\right)$. If $F^{\prime}=\left(e_{1}, e_{2}, e_{3}\right)$, then $\left\{e_{1}, e_{2}, e_{3}\right\}$ is a triangle in $M_{0} \backslash y$. As $\left\{e_{1}, e_{2}, y\right\}$ is a triangle in $M_{0} \backslash e$, we see that $\left\{e_{1}, e_{2}, e_{3}, y\right\}$ is a $U_{2,4}$-restriction in $M_{0} \backslash e$, and $\left\{e_{2}, y, x\right\}$ is a triad, so we have a contradiction. Now it follows that $F^{\prime} \neq\left(e_{1}, e_{2}, e_{3}\right)$, so $e$ and $x$ are internal elements of $F^{\prime}$. By Proposition 6.6, $x$ is a rim element of $F^{\prime}$, and $x$ and $e$ are consecutive. As $\left\{e_{1}, e_{2}, e_{3}\right\}$ is a triangle in $M_{0} \backslash y \backslash e / x$, it follows from Proposition 5.2 that $e_{1}$ is a spoke element of $F^{\prime}$. It follows that $F^{\prime}$ is either $\left(e_{1}, e_{2}, e, x, e_{3}\right)$ or $\left(e_{1}, x, e, e_{2}, e_{3}\right)$. In the first case, $\left\{e_{1}, e_{2}, e, y\right\}$ and $\left\{e_{3}, e, x, y\right\}$ are $U_{2,4}$-restrictions of $M_{0}$. If $r_{M_{0}}\left(\left\{e, x, y, e_{1}, e_{2}, e_{3}\right\}\right)>2$, then submodularity implies that $r_{M_{0}}(\{e, y\}) \leqslant 1$, which contradicts 3-connectivity. Therefore $r_{M_{0} \backslash e}\left(\left\{x, y, e_{1}, e_{2}, e_{3}\right\}\right)=2$, which quickly leads to a contradiction. We conclude that $F^{\prime}=\left(e_{1}, x, e, e_{2}, e_{3}\right)$, so $\left\{e_{1}, x, e\right\}$ and $\left\{e, e_{2}, e_{3}\right\}$ are triangles of $M_{0}$.

Because $\left\{e, e_{1}\right\}$ and $\left\{e_{3}, y\right\}$ are parallel pairs in $M_{0} / x$, we will swap the labels on $e$ and $e_{1}$, and on $e_{3}$ and $y$. Let $M^{\prime}$ be the resulting matroid, so $N$ is a minor of $M^{\prime} \backslash e / x \backslash y$, and $M^{\prime}$ has triangles $\left\{e, e_{2}, e_{3}\right\},\left\{e_{3}, x, y\right\},\left\{e, e_{1}, x\right\}$, and $\left\{e_{1}, e_{2}, y\right\}$, as well as the cocircuit $\left\{e_{1}, e_{2}, e_{3}, x\right\}$. Assume that $e$ is in a triad, $T^{*}$, of $M^{\prime}$. Orthogonality implies that $T^{*}$ contains an element from each of $\left\{e_{2}, e_{3}\right\}$ and $\left\{e_{1}, x\right\}$. By orthogonality with $\left\{e_{1}, e_{2}, y\right\}$ and $\left\{e_{3}, x, y\right\}$, and the fact that $\left\{e_{1}, e_{2}\right\}$ is not a series pair in $N$ (and therefore not in $\left.M^{\prime} \backslash e\right)$, we see that $T^{*}=\left\{e, e_{3}, x\right\}$. Therefore $\left\{e_{3}, x, y\right\}$ is a codependent triangle in $M^{\prime} \backslash e$. This contradiction shows that $M^{\prime} \backslash e$ is 3-connected, and is therefore a fan-extension of $N$.

Let $F^{\prime}$ be a fan in $M^{\prime} \backslash e$ that contains $\left(e_{1}, e_{2}, e_{3}\right)$ as a subsequence, and that has $e_{1}$ and $e_{3}$ as terminal elements. By the same arguments as before, we see that $F^{\prime}-\{x, y\}$ is a fan in $M^{\prime} \backslash e / x \backslash y$. However, $\left\{e_{1}, e_{2}, e_{3}\right\}$ is a triangle in $M^{\prime} \backslash e / x \backslash y$, so $F^{\prime}-\{x, y\}=\left(e_{1}, e_{2}, e_{3}\right)$. Assume $F^{\prime}=\left(e_{1}, e_{2}, e_{3}\right)$. Because $\left\{e_{3}, x, y\right\}$ is a triangle in $M^{\prime} \backslash e$, orthogonality shows that $\left\{e_{1}, e_{2}, e_{3}\right\}$ is also a triangle. Because $\left\{e, e_{2}, e_{3}\right\},\left\{e_{1}, e_{2}, y\right\}$, and $\left\{e, e_{1}, x\right\}$ are also triangles in $M^{\prime}$, it follows that $r_{M^{\prime}}\left(\left\{e_{1}, e_{2}, e_{3}, e, x, y\right\}\right)=2$. This means that $\left\{e_{1}, e_{2}, e_{3}\right\}$ is a parallel class in $M^{\prime} / x$, so we have a contradiction. Therefore $F^{\prime}$ is not $\left(e_{1}, e_{2}, e_{3}\right)$, so $\left\{x, y, e_{2}\right\}$ is a consecutive set in $F^{\prime}$. If $\left\{x, y, e_{2}\right\}$ is a triangle in $M^{\prime} \backslash e$, then exactly as before, we see that $r_{M^{\prime}}\left(\left\{e_{1}, e_{2}, e_{3}, e, x, y\right\}\right)=2$. Therefore $\left\{x, y, e_{2}\right\}$ is a triad of $M^{\prime} \backslash e$, and $\left\{e, e_{2}, x, y\right\}$ is a cocircuit of $M^{\prime}$, by orthogonality with $\left\{e, e_{2}, e_{3}\right\}$. Because $\left\{e_{1}, e_{2}, e_{3}, x\right\}$ is also a cocircuit, we see that $\left\{e, e_{2}, e_{3}, x\right\}$ cospans $\left\{e_{1}, e_{2}, e_{3}, e, x, y\right\}$ in $M^{\prime}$. As $\left\{e, e_{3}, y\right\}$ spans the same set, it follows that $\lambda_{M^{\prime}}\left(\left\{e_{1}, e_{2}, e_{3}, e, x, y\right\}\right) \leqslant 1$. This means that $\left|E\left(M^{\prime} \backslash e\right)\right| \leqslant 6$, and as $\left|F^{\prime}\right|=5$, it follows from Proposition 5.4 that $M^{\prime} \backslash e$ is a wheel or a whirl. This contradiction completes the proof of Lemma 6.16.

In fact, we can strengthen Lemma 6.16 further, and show that elements in $E\left(M_{0} \backslash e\right)-$ $E(N)$ are terminal elements of fans in a covering family.

Lemma 6.17. Let $M_{0}$ be isomorphic to $M$. Assume that $N$ is a minor of $M_{0}$, but that $M_{0}$ is not a fan-extension of $N$. Assume also that $M_{0} \backslash e$ is 3-connected and has $N$ as 
a minor. Let $\mathcal{F}_{e}$ be a covering family of $M_{0} \backslash e$, and let $\left(e_{1}, \ldots, e_{m}\right)$ be a fan in $\mathcal{F}_{e}$. If $1<i<m$, then $e_{i}$ is in $E(N)$.

Proof. Assume that the lemma fails. By applying Lemma 6.16 and reversing as required, we can assume that $\left(e_{1}, \ldots, e_{m}\right)$ is in $\mathcal{F}_{e}$, and that $e_{2}$ is in $E\left(M_{0} \backslash e\right)-E(N)$.

6.17.1. $e_{1}$ is in $E\left(M_{0} \backslash e\right)-E(N)$.

Proof. If $e_{2}$ is a rim element in $\left(e_{1}, \ldots, e_{m}\right)$, then we let $M^{\prime}$ be $M_{0} \backslash e$ and we let $N^{\prime}$ be $N$. Otherwise we let $M^{\prime}$ be $\left(M_{0} \backslash e\right)^{*}$ and we let $N^{\prime}$ be $N^{*}$. Thus $M^{\prime}$ is a fan-extension of $N^{\prime}$ and $\mathcal{F}_{e}$ is a covering family, while $e_{2} \in E\left(M^{\prime}\right)-E\left(N^{\prime}\right)$ is a rim element in the fan $\left(e_{1}, \ldots, e_{m}\right) \in \mathcal{F}_{e}$ of $M^{\prime}$.

Assume for a contradiction that $e_{1}$ is in $E\left(N^{\prime}\right)$. If $N^{\prime}$ is a minor of $M^{\prime} \backslash e_{2}$, then $m=4$, for otherwise $\left\{e_{3}, e_{4}, e_{5}\right\}$ is a codependent triangle in $M^{\prime} \backslash e_{2}$, violating Proposition 6.2. But if $m=4$, then $\left\{e_{1}, e_{3}, e_{4}\right\}$ are all contained in a fan in $\mathcal{F}_{N}$, and $\left\{e_{3}, e_{4}\right\}$ is a series pair in $M^{\prime} \backslash e_{2}$, which leads to a contradiction to the 3-connectivity of $N^{\prime}$. Therefore $N^{\prime}$ is a minor of $M^{\prime} / e_{2}$. If $m=4$, then $\left\{e_{1}, e_{3}, e_{4}\right\} \subseteq E\left(N^{\prime}\right)$, and $\left\{e_{1}, e_{3}\right\}$ is a parallel pair in $M^{\prime} / x_{2}$. This leads to a contradiction, so $m \geqslant 5$, meaning that Lemma 6.16 implies $e_{3} \in E\left(N^{\prime}\right)$. Now $\left\{e_{1}, e_{3}\right\}$ is a parallel pair in $M^{\prime} / e_{2}$, and we again obtain a contradiction to the 3-connectivity of $N^{\prime}$.

It follows from 6.17.1 that $m \geqslant 5$. If $e_{1}$ is a rim element of $\left(e_{1}, \ldots, e_{m}\right)$, then it is easily checked that $N$ is a minor of $M_{0} \backslash e / e_{1} \backslash e_{2}$. If $e_{1}$ is a spoke element, then $N$ is a minor of $M_{0} \backslash e \backslash e_{1} / e_{2}$. Let $M^{\prime}$ be the matroid obtained from $M_{0}$ by swapping the labels on $e_{1}$ and $e_{3}$. If $e_{1}$ is a rim element, then $\left\{e_{1}, e_{3}\right\}$ is a series pair in $M_{0} \backslash e \backslash e_{2}$, so $M^{\prime} \backslash e / e_{1} \backslash e_{2}=M_{0} \backslash e / e_{1} \backslash e_{2}$. If $e_{1}$ is a spoke element, then we can similarly show that $M^{\prime} \backslash e \backslash e_{1} / e_{2}=M_{0} \backslash e \backslash e_{1} / e_{2}$. Therefore $N$ is a minor of $M^{\prime} \backslash e / e_{1} \backslash e_{2}$ or $M^{\prime} \backslash e \backslash e_{1} / e_{2}$.

Assume that $M^{\prime}$ is not a fan-extension of $N$. Since $e_{1}$ and $e_{2}$ are not in $E(N)$, it is easy to see that

$$
\left(\mathcal{F}_{e}-\left\{\left(e_{1}, \ldots, e_{m}\right)\right\}\right) \cup\left\{\left(e_{3}, e_{2}, e_{1}, e_{4}, \ldots, e_{m}\right)\right\}
$$

is a covering family of $M^{\prime} \backslash e$. But this contradicts Lemma 6.16, as $e_{1}$ is not in $E(N)$. Therefore $M^{\prime}$ is a fan-extension of $N$. Note that this does not contradict Lemma 6.15, which would apply if $N$ were a minor of $M_{0} \backslash e / e_{2} \backslash e_{3}$ or $M_{0} \backslash e \backslash e_{2} / e_{3}$.

Let $F_{N}$ be the fan in $\mathcal{F}_{N}$ that is consistent with $\left(e_{1}, \ldots, e_{m}\right)$.

6.17.2. $e_{3}$ is in $E(N)$, but not in $F_{N}$, and therefore $e_{3}$ is contained in no fan in $\mathcal{F}_{N}$.

Proof. It follows immediately from Lemma 6.16 that $e_{3}$ is in $E(N)$. Assume that 6.17 .2 fails, so that $e_{3}$ is in $F_{N}$. Because $M^{\prime}$ is a fan-extension of $N$, there is a covering family, $\mathcal{F}^{\prime}$, of $M^{\prime}$. Let $F^{\prime}$ be the fan in $\mathcal{F}^{\prime}$ such that $F_{N}$ is consistent with $F^{\prime}$. If $e$ is in $F^{\prime}$, then it is a terminal spoke element, as $M^{\prime} \backslash e$ is isomorphic to $M \backslash e$, which is 3-connected. Therefore $F^{\prime}-e$ is a fan in $M^{\prime} \backslash e$, and so is $\left(e_{3}, e_{2}, e_{1}, e_{4}, \ldots, e_{m}\right)$. As these fans both contain the elements of $F_{N}$, it follows from Lemma 5.8 that the elements they have in common form a contiguous subsequence in both fans. In particular, this means that $\left\{e_{3}, e_{2}, e_{1}\right\}$ is contained in $F^{\prime}-e$. As this set is either a triad or a triangle in $M^{\prime} \backslash e$, 
it forms a set of three consecutive elements in $F^{\prime}-e$, and hence in $F^{\prime}$. Let $F^{\prime \prime}$ be the sequence obtained from $F^{\prime}$ by swapping the positions of $e_{1}$ and $e_{3}$. As $e_{1}$ and $e_{2}$ are not in $E(N)$, it follows that $\left(\mathcal{F}^{\prime}-\left\{F^{\prime}\right\}\right) \cup\left\{F^{\prime \prime}\right\}$ is a covering family of $M_{0}$. This contradiction to Lemma 6.11 completes the proof of 6.17.2.

6.17.3. Let $\mathcal{F}^{\prime}$ be a covering family of $M^{\prime}$. Then no fan in $\mathcal{F}^{\prime}$ contains $e_{3}$.

Proof. Assume for a contradiction that $e_{3}$ is contained in a fan in $\mathcal{F}^{\prime}$. Let $\mathcal{F}^{\prime \prime}$ be the family of fans in $M_{0}$ obtained from $\mathcal{F}^{\prime}$ by swapping the labels on $e_{1}$ and $e_{3}$. Obviously there are $\left|\mathcal{F}_{N}\right|$ pairwise disjoint fans in $\mathcal{F}^{\prime \prime}$. Since $e_{3}$ is not contained in a fan in $\mathcal{F}_{N}$ (by 6.17.2), and the same statement applies to $e_{1}$, it follows that every fan in $\mathcal{F}_{N}$ is consistent with a fan in $\mathcal{F}^{\prime \prime}$. Moreover, every element in $E\left(M^{\prime}\right)-E(N)$ is contained in a fan in $\mathcal{F}^{\prime}$, and since $e_{3}$ is contained in a fan in $\mathcal{F}^{\prime}$, and $e_{3} \in E(N)$, we conclude that every element in $E\left(M_{0}\right)-E(N)$ is contained in a fan in $\mathcal{F}^{\prime \prime}$. We have just shown that $\mathcal{F}^{\prime \prime}$ is a covering family of $M_{0}$, contradicting Lemma 6.11.

Let $\{x, y\}=\left\{e_{1}, e_{2}\right\}$, where $N$ is a minor of $M \backslash e / x \backslash y$.

6.17.4. Let $\mathcal{F}^{\prime}$ be a covering family of $M^{\prime}$. Then there is a fan in $\mathcal{F}^{\prime}$ that contains $e_{1}$ and $e_{2}$.

Proof. Let $\mathcal{F}^{\prime}$ be a covering family of $M^{\prime}$. Assume that 6.17 .4 fails, so that there are distinct fans, $F_{1}$ and $F_{2}$, in $\mathcal{F}^{\prime}$ that (respectively) contain $e_{1}$ and $e_{2}$. Note that $F_{1}$ and $F_{2}$ contain at least four elements each. If $e$ is contained in either $F_{1}$ or $F_{2}$, then it is a terminal spoke element, as $M^{\prime} \backslash e$ is 3 -connected. Therefore $F_{1}-e$ and $F_{2}-e$ are fans in $M^{\prime} \backslash e$, and these fans must each contain at least four elements, as they contain a fan in $\mathcal{F}_{N}$, and an element in $\left\{e_{1}, e_{2}\right\}$. Now $\left\{e_{1}, e_{2}, e_{3}\right\}$ is a triangle or a triad in $M^{\prime} \backslash e$, and $F_{1}-e$ and $F_{2}-e$ are fans. Since 6.17 .3 asserts that $e_{3}$ is in neither $F_{1}-e$ nor $F_{2}-e$, orthogonality requires that either $e_{1}$ and $e_{2}$ are both terminal spoke elements of $F_{1}-e$ and $F_{2}-e$, or they are both terminal rim elements. In this former case, $M^{\prime} \backslash e / x$ contains a triad that contains a parallel pair, and in the latter case, $M^{\prime} \backslash e \backslash y$ contains a triangle that contains a series pair. In either case we have a contradiction, so 6.17.4 must hold.

Let $\mathcal{F}^{\prime}$ be a covering family of $M^{\prime}$, and let $F^{\prime}$ be a fan in $\mathcal{F}^{\prime}$ that contains $e_{1}$ and $e_{2}$. Then $F^{\prime}$ contains at least five elements. If $e$ is in $F^{\prime}$, then it is a terminal spoke element, as $M^{\prime} \backslash e$ is 3 -connected, so $F^{\prime}-e$ is a fan in $M^{\prime} \backslash e$. Let $F^{\prime}-e$ be $\left(f_{1}, \ldots, f_{n}\right)$. Note that $n \geqslant 5$. Now $\left\{e_{1}, e_{2}, e_{3}\right\}$ is a triangle or a triad in $M^{\prime} \backslash e$ that intersects in $F^{\prime}-e$ in exactly the elements $e_{1}$ and $e_{2}$ (by 6.17.3). We apply Proposition 5.3 or its dual. Assume that statement (iii), (iv), or (v) holds. Then either $\left\{e_{1}, e_{2}\right\}=\left\{f_{1}, f_{n}\right\}$, or $n=5$ and $\left\{e_{1}, e_{2}\right\}=\left\{f_{2}, f_{4}\right\}$, and in any case $e_{1}$ and $e_{2}$ are both spoke elements in $F^{\prime}-e$, or both are rim elements. If both are spoke elements, $M^{\prime} \backslash e / x$ contains a triad that contains a parallel pair, and if both are rim elements, then $M^{\prime} \backslash e \backslash y$ contains a triangle that contains a series pair. In either case we have a contradiction, so statements (iii), (iv), and (v) in Proposition 5.3 cannot hold. Now, by reversing as necessary, we can assume that $\left\{e_{1}, e_{2}\right\}=\left\{f_{1}, f_{2}\right\}$. 
6.17.5. $F^{\prime}$ is either $\left(f_{1}, \ldots, f_{n}\right)$ or $\left(f_{1}, \ldots, f_{n}, e\right)$.

Proof. If $e$ is not contained in $F^{\prime}$, then $F^{\prime}=F^{\prime}-e=\left(f_{1}, \ldots, f_{n}\right)$, and we are done. Therefore assume that $e$ is a terminal spoke element of $F^{\prime}$. The only way 6.17 .5 can fail to be true is if $F^{\prime}=\left(e, f_{1}, \ldots, f_{n}\right)$, so let us assume this is the case. Then $\left\{e, e_{1}, e_{2}\right\}=\left\{e, f_{1}, f_{2}\right\}$ is a triangle of $M^{\prime}$. If $\left\{e_{1}, e_{2}, e_{3}\right\}$ is a triangle of $M^{\prime}$, then $\left\{e, e_{1}, e_{2}, e_{3}\right\}$ is a $U_{2,4}$-restriction, which is a contradiction, as $\left\{f_{1}, f_{2}, f_{3}\right\}=\left\{e_{1}, e_{2}, f_{3}\right\}$ is a triad. Similarly, if $\left\{e_{1}, e_{2}, e_{3}\right\}$ is a triad in $M^{\prime}$, then $\left\{e_{2}, e_{1}, e_{4}\right\}$ is a triangle, so $\left\{e, e_{2}, e_{1}, e_{4}\right\}$ is a $U_{2,4}$-restriction. This again leads to a contradiction. Therefore 6.17 .5 holds.

6.17.6. $\left\{e, e_{1}, e_{2}, e_{3}\right\}$ is a cocircuit in $M^{\prime}$.

Proof. Assume that $\left\{e_{1}, e_{2}, e_{3}\right\}$ is a triangle in $M^{\prime} \backslash e$, and hence in $M^{\prime}$. If $\left\{f_{1}, f_{2}, f_{3}\right\}$ is a triangle in $M^{\prime}$, then $\left\{e_{3}, f_{1}, f_{2}, f_{3}\right\}$ is a $U_{2,4}$-restriction in $M^{\prime}$, and $\left\{f_{2}, f_{3}, f_{4}\right\}$ is a triad. As this is a contradiction, it follows that $\left\{f_{1}, f_{2}, f_{3}\right\}$ is a triad in $M^{\prime}$. Let $F^{\prime}+e_{3}$ be the sequence obtained from $F^{\prime}$ by appending $e_{3}$ to the beginning. Then $F^{\prime}+e_{3}$ is a fan in $M^{\prime}$, and it follows from 6.17.2 and 6.17.3 that $\left(\mathcal{F}^{\prime}-\left\{F^{\prime}\right\}\right) \cup\left\{F^{\prime}+e_{3}\right\}$ is a covering family of $M^{\prime}$. But this contradicts 6.17.3, so we conclude that $\left\{e_{1}, e_{2}, e_{3}\right\}$ is a triad in $M^{\prime} \backslash e$.

To conclude the proof of 6.17.6, assume that $\left\{e_{1}, e_{2}, e_{3}\right\}$ is a triad in $M^{\prime}$. If $\left\{f_{1}, f_{2}, f_{3}\right\}$ is a triad in $M^{\prime}$, then $\left\{e_{3}, f_{1}, f_{2}, f_{3}\right\}$ is a $U_{2,4}$-corestriction in $M^{\prime}$, which is a contradiction as $\left\{f_{2}, f_{3}, f_{4}\right\}$ is a triangle. Therefore $\left\{f_{1}, f_{2}, f_{3}\right\}$ is a triangle in $M^{\prime}$. We again let $F^{\prime}+e_{3}$ be obtained from $F^{\prime}$ by appending $e_{3}$ to the beginning. This leads to a contradictory covering family, just as in the previous paragraph. Therefore $\left\{e, e_{1}, e_{2}, e_{3}\right\}$ is a cocircuit in $M$.

As $e$ is in $E\left(M^{\prime}\right)-E(N)$, there is a fan, $F_{e}$, in $\mathcal{F}^{\prime}$, that contains $e$. Then $F_{e}$ contains at least four elements. As $M^{\prime} \backslash e$ is 3-connected, it follows that $e$ is a terminal spoke element in $F_{e}$. Let $\{e, u, v\}$ be the triangle contained in $F_{e}$ that contains $e$. Orthogonality between the triangle $\{e, u, v\}$ and the cocircuit $\left\{e, e_{1}, e_{2}, e_{3}\right\}$ shows that either $e_{1}, e_{2}$, or $e_{3}$ is in $\{u, v\}$. The last case is impossible by 6.17 .3 , so $e_{1}$ or $e_{2}$ is in $\{u, v\}$. This means that $F_{e}$ and $F^{\prime}$ cannot be disjoint fans, so $F^{\prime}=\left(f_{1}, \ldots, f_{n}, e\right)$, by 6.17.5. However it now follows that $\left\{e_{1}, e_{2}\right\}=\left\{f_{1}, f_{2}\right\}$, and $\{u, v\}=\left\{f_{n-1}, f_{n}\right\}$. Thus $F^{\prime}$ can contain at most four elements, which is impossible, as it contains $e, e_{1}, e_{2}$, and a fan in $\mathcal{F}_{N}$. This completes the proof of Lemma 6.17.

Lemma 6.14 supplies us with a matroid $M_{0}$, which we now relabel as $M$, such that $M$ has $N$ as a minor, but is not a fan-extension of $N$. By replacing $M, N$, and $\mathcal{M}$ with their duals as necessary, we can assume that $M \backslash e$ is 3 -connected and has $N$ as a minor. The minimality of $M$ means that $M \backslash e$ is a fan-extension of $N$.

Lemma 6.18. Let $\mathcal{F}_{e}$ be a covering family of $M \backslash e$, and assume that $\left(e_{1}, \ldots, e_{m}\right)$ is a fan in $\mathcal{F}_{e}$. Assume also that $e_{1}$ is not in $E(N)$. If $e_{1}$ is a spoke element of $\left(e_{1}, \ldots, e_{m}\right)$, then $M \backslash e \backslash e_{1}$ is 3-connected and has $N$ as a minor. If $e_{1}$ is a rim element, then $M \backslash e / e_{1}$ is 3-connected and has $N$ as a minor. 
Proof. If $e_{1}$ is a rim element, then let $M^{\prime}=M \backslash e$ and let $N^{\prime}=N$. Otherwise, let $M^{\prime}=(M \backslash e)^{*}$ and let $N^{\prime}=N^{*}$. Thus, in either case, we assume that $\mathcal{F}_{e}$ is a covering family of $M^{\prime}$ relative to $N^{\prime}$ and $\mathcal{F}_{N}$, that $\left(e_{1}, \ldots, e_{m}\right)$ is a fan in $\mathcal{F}_{e}$, and $e_{1}$ is a rim element that is not in $E\left(N^{\prime}\right)$. Certainly $M^{\prime} / e_{1}$ has $N^{\prime}$ as a minor, or else $M^{\prime} \backslash e_{1}$ has $N^{\prime}$ as a minor and $\left\{e_{2}, e_{3}, e_{4}\right\}$ as a codependent triangle, contradicting Proposition 6.2. Assume $M^{\prime} / e_{1}$ is not 3 -connected. Then $e_{1}$ is contained in a triangle, $T$, of $M^{\prime}$. Orthogonality with the triad $\left\{e_{1}, e_{2}, e_{3}\right\}$ shows that $T$ contains $e_{2}$ or $e_{3}$. Note that $T \nsubseteq\left\{e_{1}, \ldots, e_{m}\right\}$, for otherwise Proposition 5.6 implies $T=\left\{e_{1}, e_{2}, e_{3}\right\}$, and thus $T$ is a triad and a triangle in $M^{\prime}$. Let $x$ be the element in $T-\left\{e_{1}, e_{2}, e_{3}\right\}$.

Assume that $e_{2}$ is in $T$. Since $e_{2}$ is in $E\left(N^{\prime}\right)$ by Lemma 6.17 , and $\left\{e_{2}, x\right\}$ is a parallel pair in $M^{\prime} / e_{1}$, it follows that $N^{\prime}$ is a minor of $M^{\prime} / e_{1} \backslash x$. The definition of a covering family means there is a fan in $\mathcal{F}_{e}$ that contains $x$. Let $F_{x}$ be this fan. Then $F_{x}$ contains at least four elements. Orthogonality with $T$ shows that $x$ is a terminal spoke element of $F_{x}$. Since $x$ is not in $E\left(N^{\prime}\right)$, it now follows that $\left(\mathcal{F}_{e}-\left\{\left(e_{1}, \ldots, e_{m}\right), F_{x}\right\}\right) \cup\left\{\left(x, e_{1}, \ldots, e_{m}\right), F_{x}-x\right\}$ is a covering family in $M^{\prime}$, and now we have a contradiction to Lemma 6.17 as $x, e_{1} \notin E\left(N^{\prime}\right)$. Therefore $T=\left\{e_{1}, e_{3}, x\right\}$.

Because $x$ is not in $\left(e_{1}, \ldots, e_{m}\right)$, we see that $m=4$, as otherwise we violate orthogonality between $T$ and $\left\{e_{3}, e_{4}, e_{5}\right\}$. Hence $\left(e_{2}, e_{3}, e_{4}\right)$ or its reversal is in $\mathcal{F}_{N}$. Let $M^{\prime \prime}$ be obtained from $M^{\prime}$ by swapping labels on $e_{3}$ and $x$. Thus $\left(e_{3}, e_{1}, x, e_{2}, e_{4}\right)$ is a fan of $M^{\prime \prime}$. As $\left\{e_{3}, x\right\}$ is a parallel pair in $M^{\prime} / e_{1}$, we see that $M^{\prime \prime}$ has $N^{\prime}$ as a minor. Since $\left|E\left(M^{\prime \prime}\right)\right|=\left|E\left(M^{\prime}\right)\right|<|E(M)|$, it follows that $M^{\prime \prime}$ is a fan-extension of $N^{\prime}$. Therefore there is a fan of $M^{\prime \prime}$ that contains $\left(e_{2}, e_{3}, e_{4}\right)$ as a subsequence. Let this fan be $F$. By comparing $F$ with $\left(e_{3}, e_{1}, x, e_{2}, e_{4}\right)$ in $M^{\prime \prime}$ using Lemma 5.8, we see that there is a contiguous subsequence of $F$ using the elements $\left\{e_{3}, e_{1}, x, e_{2}, e_{4}\right\}$. We apply Proposition 5.6 and its dual to $\left\{e_{3}, e_{1}, x\right\},\left\{e_{1}, x, e_{2}\right\}$, and $\left\{x, e_{2}, e_{4}\right\}$ and see that $F$ contains $\left(e_{3}, e_{1}, x, e_{2}, e_{4}\right)$ as a contiguous subsequence, which contradicts the definition of $F$. Therefore $M^{\prime} / e_{1}$ is 3 -connected, and this completes the proof.

Lemma 6.19. Let $\mathcal{F}_{e}$ be a covering family of $M \backslash e$, and assume that $\left(e_{1}, \ldots, e_{m}\right)$ is a fan in $\mathcal{F}_{e}$. Assume also that $e_{1}$ is not in $E(N)$. If $e_{1}$ is a rim element of $\left(e_{1}, \ldots, e_{m}\right)$, then assume that there is no triangle of $M$ that contains $\left\{e_{1}, e\right\}$. Let $\left(e_{s}, \ldots, e_{t}\right)$ be a minimal contiguous subsequence of $\left(e_{1}, \ldots, e_{m}\right)$ such that a fan, $F_{N} \in \mathcal{F}_{N}$ is consistent with $\left(e_{s}, \ldots, e_{t}\right)$. Then $2 \leqslant s<s+2 \leqslant t \leqslant m$ and $F_{N}$ contains $e_{s}$ and $e_{t}$. Moreover, $F_{N}$ is equal to $\left(e_{s}, \ldots, e_{t}\right)$ or its reversal, and is a fan of $M$. However, $\left(e_{1}, \ldots, e_{t}\right)$ is not a fan in $M$.

Proof. Since $\left(e_{1}, \ldots, e_{m}\right)$ is a fan in a covering family, there is a fan in $\mathcal{F}_{N}$ that is consistent with $\left(e_{1}, \ldots, e_{m}\right)$. Let $e_{s}$ be the first element in this fan, and let $e_{t}$ be the last. Then $2 \leqslant s<s+2 \leqslant t \leqslant m$ because each fan contains at least three elements, and $e_{1} \notin E(N)$. By Lemma 6.17 it follows that every element in $\left\{e_{s}, \ldots, e_{t}\right\}$ is in $E(N)$. Since $N$ is 3-connected, $\left(e_{s}, \ldots, e_{t}\right)$ is a fan of $N$. As $F_{N}$ is also a fan of $N$, we apply Lemma 5.8 and deduce that $F_{N}$ and $\left(e_{s}, \ldots, e_{t}\right)$ use the same set of elements. Since $F_{N}$ is consistent with $\left(e_{s}, \ldots, e_{t}\right)$, this means that $F_{N}$ is equal to $\left(e_{s}, \ldots, e_{t}\right)$ or its reversal.

If $e_{1}$ is a spoke element of $\left(e_{1}, \ldots, e_{m}\right)$, let $M^{\prime}$ be $M \backslash e_{1}$. In this case, $M^{\prime}$ is 3-connected, 
since $M^{\prime} \backslash e$ is 3-connected by Lemma 6.18. If $e_{1}$ is a rim element, then let $M^{\prime}$ be $M / e_{1}$. In this case also, $M^{\prime} \backslash e$ is 3-connected, and the hypotheses imply that $e$ is not in a parallel pair in $M^{\prime}$. Therefore $M^{\prime}$ is 3 -connected in either case, and $M^{\prime}$ has $N$ as a minor. As $\left|E\left(M^{\prime}\right)\right|<|E(M)|$, it follows that $M^{\prime}$ is a fan-extension of $N$.

Let $F$ be a fan of $M^{\prime}$ such that $\left(e_{s}, \ldots, e_{t}\right)$ is consistent with $F$. If $e$ is in $F$, then it is a terminal spoke element, as $M^{\prime} \backslash e$ is 3 -connected, so in this case $F-e$ is a fan in $M^{\prime} \backslash e$. In fact, $F-e$ is a fan of $M^{\prime} \backslash e$ whether or not $e$ is in $F$. Since $\left(e_{s}, \ldots, e_{t}\right)$ is also a fan in $M^{\prime} \backslash e$, by Lemma 5.8, there is a contiguous subsequence of $F-e$ using the elements $\left\{e_{s}, \ldots, e_{t}\right\}$. Since $\left(e_{s}, \ldots, e_{t}\right)$ is consistent with $F-e$, this means $\left(e_{s}, \ldots, e_{t}\right)$ is a contiguous subsequence of $F-e$. Hence $\left(e_{s}, \ldots, e_{t}\right)$ is a fan in $M^{\prime}$.

Assume $\left(e_{s}, \ldots, e_{t}\right)$ is not a fan in $M$. If $e_{1}$ is a spoke element in $\left(e_{1}, \ldots, e_{m}\right)$, then $M^{\prime}=M \backslash e_{1}$, so there is a rim element, $e_{i}$, of $\left(e_{s}, \ldots, e_{t}\right)$, such that $s \leqslant i \leqslant t-2$, and $\left\{e_{1}, e_{i}, e_{i+1}, e_{i+2}\right\}$ is a cocircuit of $M$. But in this case $e_{1}$ is in the closure and coclosure of $\left(e_{2}, \ldots, e_{m}\right)$ in $M \backslash e$. Hence $\left(e_{1}, \ldots, e_{m}\right)$ is 2-separating in $M \backslash e$, so Proposition 5.4 implies $M \backslash e$ is a wheel or a whirl, a contradiction. The argument when $e_{1}$ is a rim element in $\left(e_{1}, \ldots, e_{m}\right)$ is similar: In this case $M^{\prime}=M / e_{1}$, so there is a spoke element, $e_{i}$, in $\left(e_{s}, \ldots, e_{t}\right)$, such that $\left\{e_{1}, e_{i}, e_{i+1}, e_{i+2}\right\}$ is a circuit of $M$. Thus $e_{1}$ is in the closure and coclosure of $\left(e_{2}, \ldots, e_{m}\right)$ in $M \backslash e$, so we can deduce that $M \backslash e$ is a wheel or a whirl, contradicting Proposition 6.1. From this contradiction we see that $\left(e_{s}, \ldots, e_{t}\right)$ is a fan of $M$.

To complete the proof, we will assume that $\left(e_{1}, \ldots, e_{t}\right)$ is a fan of $M$, and deduce a contradiction. This assumption means that $\left(e_{2}, \ldots, e_{t}\right)$ is a fan in $M^{\prime}$.

6.19.1. There is a covering family of $M^{\prime}$ containing a fan that has $\left(e_{2}, \ldots, e_{t}\right)$ as a contiguous subsequence.

Proof. Let $\mathcal{F}$ be a covering family of $M^{\prime}$. Then $\mathcal{F}$ contains a fan, $F$, such that $\left(e_{s}, \ldots, e_{t}\right)$ is consistent with $F$. As $\left(e_{s}, \ldots, e_{t}\right)$ is a fan in $M^{\prime}$, we can use Lemma 5.8 to show that $\left(e_{s}, \ldots, e_{t}\right)$ is a contiguous subsequence of $F$. Assume that $F$ contains $\left(e_{i}, \ldots, e_{t}\right)$ as a contiguous subsequence, where $2 \leqslant i \leqslant s$, and $\mathcal{F}$ and $F$ have been chosen so that $i$ is as small as possible. If $i=2$, then 6.19 .1 is already proved, so we assume $2<i$. Let $F=\left(f_{1}, \ldots, f_{n}\right)$, where $\left(e_{i}, \ldots, e_{t}\right)=\left(f_{j}, \ldots, f_{j+t-i}\right)$.

Assume $j>1$. Then $f_{j-1} \neq e_{i-1}$, for otherwise $\left(e_{i-1}, \ldots, e_{t}\right)$ is a contiguous subsequence of $F$, and the minimality of $i$ is contradicted. Since $\left\{f_{j}, f_{j+1}, f_{j+2}\right\}=\left\{e_{i}, e_{i+1}, e_{i+2}\right\}$ is not a triad and a triangle, $f_{j}=e_{i}$ is a spoke element in both $F$ and $\left(e_{2}, \ldots, e_{m}\right)$, or a rim element in both. Now $\left\{f_{j-1}, f_{j}, f_{j+1}\right\}$ and $\left\{e_{i-1}, e_{i}, e_{i+1}\right\}$ are distinct triads or distinct triangles that intersect in two elements, and their union is a $U_{2,4}$-corestriction or restriction of $M^{\prime}$ that intersects a triangle or triad. This contradiction shows that $j=1$.

Now $\left(e_{i-1}, f_{1}, \ldots, f_{n}\right)$ is a fan of $M^{\prime}$. Let $F^{\prime}$ be this fan. Note that because $i-1<s$, the element $e_{i-1}$ is not contained in the fan $\left(e_{s}, \ldots, e_{t}\right)$, and since it is contained in $\left(e_{1}, \ldots, e_{m}\right) \in \mathcal{F}_{e}$, the definition of a covering family tells us that $e_{i-1}$ is contained in no fan in $\mathcal{F}_{N}$. If $e_{i-1}$ is in no fan in $\mathcal{F}$, then $(\mathcal{F}-\{F\}) \cup\left\{F^{\prime}\right\}$ is a covering family in $M^{\prime}$. If $e_{i-1}$ is in a fan, $F^{\prime \prime} \in \mathcal{F}$, then it is a terminal element of $F^{\prime \prime}$ by orthogonality with $\left\{e_{i-1}, e_{i}, e_{i+1}\right\}$, so $\left(\mathcal{F}-\left\{F, F^{\prime \prime}\right\}\right) \cup\left\{F^{\prime}, F^{\prime \prime}-e_{i-1}\right\}$ is a covering family of $M^{\prime}$. In either case 
we have constructed a covering family of $M^{\prime}$ that contains the fan $F^{\prime}$, so the minimality of $i$ is contradicted. This completes the proof of 6.19.1.

Now we assume $\mathcal{F}$ is a covering family of $M^{\prime}$, and $\mathcal{F}$ contains a fan, $F$, that has $\left(e_{2}, \ldots, e_{t}\right)$ as a contiguous subsequence. Let $F=\left(f_{1}, \ldots, f_{n}\right)$, where $\left(e_{2}, \ldots, e_{t}\right)=$ $\left(f_{j}, \ldots, f_{j+t-2}\right)$. Assume $j>1$. If $e_{1}$ is a rim element of $\left(e_{1}, \ldots, e_{m}\right)$, then $\left\{e_{2}, e_{3}, e_{4}\right\}$ is a triangle in $M$, and hence in $M^{\prime}=M / e_{1}$. Therefore $e_{2}$ is a spoke element in $\left(f_{j}, \ldots, f_{j+t-2}\right)$, so $\left\{f_{j-1}, f_{j}, f_{j+1}\right\}$ is a triad of $M^{\prime}$ and therefore in $M$. Now $\left\{e_{1}, f_{j-1}, f_{j}, f_{j+1}\right\}$ is a $U_{2,4}$-corestriction of $M$ that intersects a triangle. If $e_{1}$ is a spoke element, we reach a similar contradiction. Therefore $j=1$. But this means $\left(e_{1}, f_{1}, \ldots, f_{n}\right)$ is a fan of $M$, and $M$ is obtained from $M^{\prime}$ by a fan-lengthening move on $F$. This implies $M$ is a fan-extension of $N$, so we have a contradiction that completes the proof of Lemma 6.19.

Now we fix $\mathcal{F}_{e}$ to be a covering family of $M \backslash e$. Since we have assumed $|E(M)|-$ $|E(N)|>2$, it follows that $M \backslash e \neq N$. Any element in $E(M \backslash e)-E(N)$ is a terminal element of a fan in $\mathcal{F}_{e}$, by Lemma 6.17.

Lemma 6.20. If $\left(e_{1}, \ldots, e_{m}\right)$ is a fan in $\mathcal{F}_{e}$ with the property that $e_{1} \notin E(N)$, then $e_{1}$ is a rim element, and $\left\{e_{1}, e\right\}$ is contained in a triangle of $M$.

Proof. If the lemma fails, then there is a fan $\left(e_{1}, \ldots, e_{m}\right)$ in $\mathcal{F}_{e}$ such that $e_{1}$ is not in $E(N)$, and either $e_{1}$ is a spoke element, or $e_{1}$ is a rim element of $\left(e_{1}, \ldots, e_{m}\right)$ and there is no triangle of $M$ containing $\left\{e_{1}, e\right\}$.

There are indices $p$ and $q$ such that $2 \leqslant p<p+2 \leqslant q \leqslant m$, and there is a fan in $\mathcal{F}_{N}$ consistent with $\left(e_{p}, \ldots, e_{q}\right)$ that contains $e_{p}$ and $e_{q}$. Now Lemma 6.19 applies, so $\left(e_{p}, \ldots, e_{q}\right)$ or its reversal is in $\mathcal{F}_{N}$, and is a fan of $M$. However, $\left(e_{1}, \ldots, e_{q}\right)$ is not a fan of $M$. Since it is a fan in $M \backslash e$, there is some $j \in\{1, \ldots, p-1\}$ such that $\left\{e, e_{j}, e_{j+1}, e_{j+2}\right\}$ is a cocircuit of $M$.

Let $M^{\prime}$ be $M \backslash e_{1}$ if $e_{1}$ is a spoke element, and let it be $M / e_{1}$ otherwise. Lemma 6.18 implies that $M^{\prime} \backslash e$ is 3 -connected with $N$ as a minor. Because the hypotheses imply $M^{\prime}$ has no parallel pair containing $e$, we now see that $M^{\prime}$ is 3 -connected. Therefore $M^{\prime}$ is a fan-extension of $N$.

As $|E(M)|-|E(N)|>2$, we can let $u$ be an element in $E\left(M^{\prime} \backslash e\right)-E(N)$. Either $u$ belongs to a fan in $\mathcal{F}_{e}$ that is distinct, and hence disjoint, from $\left(e_{1}, \ldots, e_{m}\right)$, or, by Lemma 6.17, $u=e_{m}$. The analyses in the two cases are similar, so we combine them. If necessary we can reverse the fan that contains $u$, and assume that we are in one of the following situations.

(I) $\left(x_{1}, \ldots, x_{n}\right)$ is a fan in $\mathcal{F}_{e}$ that is disjoint from $\left(e_{1}, \ldots, e_{m}\right)$, and $x_{1}$ is not in $E(N)$.

(II) $e_{m}$ is not in $E(N)$. In this case we let $\left(x_{1}, \ldots, x_{n}\right)$ be $\left(e_{m}, \ldots, e_{2}\right)$.

Note that in either of these cases, $\left(x_{1}, \ldots, x_{n}\right)$ is a fan in $M \backslash e$ and in $M^{\prime} \backslash e$, and $x_{1}$ is not in $E(N)$. By taking a minimal contiguous subsequence of $\left(x_{1}, \ldots, x_{n}\right)$ such that a fan in $\mathcal{F}_{N}$ is consistent with the subsequence, we see there are integers $s$ and $t$ such that $2 \leqslant s<s+2 \leqslant t \leqslant n$ and such that some fan in $\mathcal{F}_{N}$ contains $x_{s}$ and $x_{t}$ and is consistent with $\left(x_{s}, \ldots, x_{t}\right)$. 
6.20.1. Either $\left(x_{s}, \ldots, x_{t}\right)$ or its reversal is in $\mathcal{F}_{N}$. Moreover, $\left(x_{s}, \ldots, x_{t}\right)$ is a fan in $M$, but $\left(x_{1}, \ldots, x_{t}\right)$ is not.

Proof. We first show that $\left(x_{s}, \ldots, x_{t}\right)$ is a fan in $M$. In Case (II), $\left(x_{s}, \ldots, x_{t}\right)$ is equal to $\left(e_{q}, \ldots, e_{p}\right)$, and we have already noted this is a fan in $M$. Therefore we assume Case (I) holds. We can apply Lemma 6.19 to $\left(x_{1}, \ldots, x_{n}\right)$, unless $x_{1}$ is a rim element and $\left\{x_{1}, e\right\}$ is contained in a triangle of $M$. If Lemma 6.19 does apply, then it tells us that $\left(x_{s}, \ldots, x_{t}\right)$ is a fan of $M$. Therefore we assume that $x_{1}$ is a rim element, and $\left\{x_{1}, e\right\}$ is contained in a triangle. Orthogonality with the cocircuit $\left\{e, e_{j}, e_{j+1}, e_{j+2}\right\}$ shows that $\left\{x_{1}, e, e_{k}\right\}$ is a triangle of $M$ for some $k \in\{j, j+1, j+2\}$. Because $\left(x_{s}, \ldots, x_{t}\right)$ is a fan in $M \backslash e$, it follows that if it is not a fan of $M$, then there is a cocircuit of $M$ that contains $e$ and three consecutive elements from $\left(x_{s}, \ldots, x_{t}\right)$. This violates orthogonality with $\left\{x_{1}, e, e_{k}\right\}$ as $s \geqslant 2$. Hence $\left(x_{s}, \ldots, x_{t}\right)$ is a fan of $M$ in any case.

Next we show that $\left(x_{s}, \ldots, x_{t}\right)$ or its reversal is in $\mathcal{F}_{N}$. Since $x_{s}$ and $x_{t}$ are in $E(N)$, Lemma 6.17 implies $\left\{x_{s}, \ldots, x_{t}\right\} \subseteq E(N)$. It follows that $\left(x_{s}, \ldots, x_{t}\right)$ is a fan of $N$. Some fan in $\mathcal{F}_{N}$ is consistent with $\left(x_{s}, \ldots, x_{t}\right)$ and contains $x_{s}$ and $x_{t}$. It follows easily from Lemma 5.8 that this fan must be either $\left(x_{s}, \ldots, x_{t}\right)$ or its reversal.

Finally we show that $\left(x_{1}, \ldots, x_{t}\right)$ is not a fan of $M$. We can apply Lemma 6.19, unless $x_{1}$ is a rim element of $\left(x_{1}, \ldots, x_{n}\right)$ and $\left\{x_{1}, e\right\}$ is contained in a triangle of $M$. If we can apply Lemma 6.19 , then it tells us that $\left(x_{1}, \ldots, x_{t}\right)$ is not a fan in $M$, as desired. Therefore we assume $x_{1}$ is a rim element, and $\left\{x_{1}, e\right\}$ is contained in a triangle, $T$, of $M$. If $\left\{x_{1}, x_{2}, x_{3}\right\}$ is not a triad of $M$, then $\left(x_{1}, \ldots, x_{t}\right)$ is not a fan of $M$, so we are done. Therefore we assume $\left\{x_{1}, x_{2}, x_{3}\right\}$ is a triad of $M$. Orthogonality shows that $T$ is $\left\{e, x_{1}, x_{2}\right\}$ or $\left\{e, x_{1}, x_{3}\right\}$.

If Case (I) holds, then we have a violation of orthogonality between $T$ and the cocircuit $\left\{e, e_{j}, e_{j+1}, e_{j+2}\right\}$. Therefore Case (II) holds, so $\left(x_{1}, x_{2}, x_{3}\right)=\left(e_{m}, e_{m-1}, e_{m-2}\right)$. Orthogonality between $\left\{e, e_{j}, e_{j+1}, e_{j+2}\right\}$ and $T$ requires that $j+2 \geqslant m-2$. Recall that $j \leqslant p-1$. Certainly $p+2 \leqslant q$, as $\left(e_{p}, \ldots, e_{q}\right)$ or its reversal is in $\mathcal{F}_{N}$. Because $e_{q}$ is in $E(N)$, but $e_{m}$ is not, it follows that $q \leqslant m-1$. Putting these together, we see that $j+2 \leqslant p+1 \leqslant q-1 \leqslant m-2$, so $j+2=m-2$, and equality holds throughout this expression. Again applying orthogonality between $\left\{e, e_{j}, e_{j+1}, e_{j+2}\right\}$ and $T$, we see that $T=\left\{e, x_{1}, x_{3}\right\}=\left\{e, e_{m}, e_{m-2}\right\}$. As $p=m-3$ and $q=m-1$, this shows that $\left(x_{s}, \ldots, x_{t}\right)=\left(e_{m-1}, e_{m-2}, e_{m-3}\right)$ and $\left(x_{2}, x_{3}, x_{4}\right)=\left(e_{m-1}, e_{m-2}, e_{m-3}\right)$ or its reversal is in $\mathcal{F}_{N}$. If $\left(x_{1}, \ldots, x_{t}\right)=\left(e_{m}, e_{m-1}, e_{m-2}, e_{m-3}\right)$ is not a fan in $M$, then we are done, so we assume it is a fan in $M$. Therefore $\left(x_{1}, x_{2}, x_{3}, x_{4}\right)$ is a fan in $M^{\prime}$ also.

Because $x_{1}$ is not in $E(N)$, it follows easily that $N$ is a minor of $M^{\prime} \backslash e / x_{1}$. Let $M^{\prime \prime}$ be the matroid obtained from $M^{\prime}$ by swapping labels on $e$ and $x_{3}$. Since $\left\{e, x_{3}\right\}$ is a parallel pair in $M^{\prime} / x_{1}, M^{\prime \prime}$ has $N$ as a minor. Moreover $M^{\prime \prime}$ is 3-connected since $M^{\prime}$ is, and $\left|E\left(M^{\prime \prime}\right)\right|=\left|E\left(M^{\prime}\right)\right|<|E(M)|$, so $M^{\prime \prime}$ is a fan-extension of $N$. Let $F$ be a fan of $M^{\prime \prime}$ such that $\left(x_{2}, x_{3}, x_{4}\right)$ is consistent with $F$. Because $M^{\prime \prime} \backslash x_{3}$ is isomorphic to $M^{\prime} \backslash e$, and is therefore 3 -connected, $x_{3}$ is contained in no triad in $F$. If $F$ contains at least four elements, then this means that $x_{3}$ is a terminal element of $F$, which is impossible as $\left(x_{2}, x_{3}, x_{4}\right)$ is consistent with $F$. Therefore $F$ contains exactly three elements, so $\left\{x_{2}, x_{3}, x_{4}\right\}$ is a triangle of $M^{\prime \prime}$. As $\left\{x_{1}, x_{2}, e\right\}$ is a triad of $M^{\prime \prime}$, we have a contradiction to orthogonality. 
Applying 6.20.1, we let $C_{x}^{*}$ be a cocircuit of $M$ of the form $\left\{e, x_{k}, x_{k+1}, x_{k+2}\right\}$, where $x_{k}$ is a rim element of $\left(x_{1}, \ldots, x_{t}\right)$ in $M \backslash e$, and $1 \leqslant k \leqslant s-1$.

6.20.2. $C_{x}^{*}$ is a cocircuit of $M^{\prime}$.

Proof. Assume Case (I) holds. If $M^{\prime}=M / e_{1}$, then $C_{x}^{*}$ is a cocircuit in $M^{\prime}$, as $e_{1}$ is not in $C_{x}^{*}$. On the other hand, if $M^{\prime}=M \backslash e_{1}$, then $\left\{e_{1}, e_{2}, e_{3}\right\}$ is a triangle disjoint from $\left\{x_{1}, \ldots, x_{n}, e\right\}$, so $C_{x}^{*}$ is a cocircuit of $M^{\prime}$. Now assume Case (II) holds. Note $C_{x}^{*}-e \subseteq\left\{x_{1}, \ldots, x_{s+1}\right\} \subseteq\left\{e_{m}, \ldots, e_{3}\right\}$, so $e_{1} \notin C_{x}^{*}$. Therefore, if $M^{\prime}=M / e_{1}$, then $C_{x}^{*}$ is a cocircuit of $M^{\prime}$. If $M^{\prime}=M \backslash e_{1}$, then $C_{x}^{*}$ is a cocircuit of $M^{\prime}$, for otherwise $e_{1}$ is in the coclosure and closure of $\left\{e_{2}, \ldots, e_{m}\right\}$ in $M \backslash e$. This implies $\left\{e_{1}, \ldots, e_{m}\right\}$ is 2-separating in $M \backslash e$, so $M \backslash e$ is a wheel or whirl, a contradiction.

Recall that $M^{\prime}$ is a fan-extension of $N$. Let $F_{e}$ be the fan containing $e$ in a covering family of $M^{\prime}$. As $M^{\prime} \backslash e$ is 3-connected, $e$ is a terminal spoke element of $F_{e}$. Let $(e, x, y, z)$ be the initial four elements of $F_{e}$, so $\{e, x, y\}$ is a triangle of $M^{\prime}$ and $\{x, y, z\}$ is a triad.

6.20.3. $\{x, y, z\} \subseteq E(N)$.

Proof. This follows from Lemma 6.17 if $\left|F_{e}\right|>4$, and from the fact that $F_{e}$ contains $e$ and a fan in $\mathcal{F}_{N}$ otherwise.

Now $\{e, x, y\}$ is a triangle in $M^{\prime}$, and $C_{x}^{*}$ is a cocircuit, so orthogonality requires that $\left(C_{x}^{*}-e\right) \cap\{x, y\} \neq \emptyset$. Thus $\{x, y, z\}$ is a triad of $M^{\prime} \backslash e$ that intersects the fan $\left(x_{1}, \ldots, x_{n}\right)$.

As $\{x, y, z\}$ is contained in $E(N)$ and $x_{1}$ is not, we see that $x_{1} \notin\{x, y, z\}$. Furthermore, any element in $C_{x}^{*} \cap\{x, y\}$ is not equal to $x_{n}$, since $C_{x}^{*}-e$ is contained in $\left\{x_{1}, \ldots, x_{s+1}\right\} \subseteq$ $\left\{x_{1}, \ldots, x_{n-1}\right\}$. This means that if $\{x, y, z\}$ intersects $\left\{x_{1}, \ldots, x_{n}\right\}$ in exactly one element, then it is a triad of $M^{\prime} \backslash e$ that intersects the fan $\left(x_{1}, \ldots, x_{n}\right)$ in a single, internal, element, violating orthogonality. Therefore $\left|\{x, y, z\} \cap\left\{x_{1}, \ldots, x_{n}\right\}\right| \geqslant 2$.

6.20.4. $\left|\{x, y, z\} \cap\left\{x_{1}, \ldots, x_{n}\right\}\right| \neq 2$.

Proof. Assume $\left|\{x, y, z\} \cap\left\{x_{1}, \ldots, x_{n}\right\}\right|=2$. We apply the dual of Proposition 5.3 in $M^{\prime} \backslash e$ to $\{x, y, z\}$ and the fan $\left(x_{1}, \ldots, x_{n}\right)$. Since $x_{1}$ is not in $\{x, y, z\}$, statement (i) or (iii) in Proposition 5.3 cannot apply. By the same reasoning, if statement (v) holds, then $n=5$. In summary, either $\{x, y, z\}$ intersects $\left\{x_{1}, \ldots, x_{n}\right\}$ in $\left\{x_{n-1}, x_{n}\right\}$, or $n \leqslant 5, x_{1}$ is a rim element, and $\{x, y, z\}$ intersects $\left\{x_{1}, \ldots, x_{n}\right\}$ in $\left\{x_{2}, x_{4}\right\}$.

Assume $\{x, y, z\} \cap\left\{x_{1}, \ldots, x_{n}\right\}=\left\{x_{n-1}, x_{n}\right\}$. Then Proposition 5.3 implies $x_{n}$ is a spoke element in $M^{\prime} \backslash e$. Since $C_{x}^{*}-e=\left\{x_{k}, x_{k+1}, x_{k+2}\right\}$ contains $x$ or $y$, and $x_{k+2}$ is a rim element, it follows that $C_{x}^{*}-e=\left\{x_{n-3}, x_{n-2}, x_{n-1}\right\}$. This implies $s=n-2$, so $\left(x_{n-2}, x_{n-1}, x_{n}\right)$ or its reversal is in $\mathcal{F}_{N}$. Since $F_{e}$ contains $(e, x, y, z)$ as a contiguous subsequence, it therefore contains an element of $\left(x_{n-2}, x_{n-1}, x_{n}\right)$. As $F_{e}$ is in a covering family of $M^{\prime}$, this means that $\left(x_{n-2}, x_{n-1}, x_{n}\right)$ is consistent with $F_{e}$. However, $x_{n-2} \notin$ $\{x, y, z\}$, and $x_{n-1} \in\{x, y\}$ as $\left(C_{x}^{*}-e\right) \cap\{x, y\} \neq \emptyset$. Since $\left(x_{n-2}, x_{n-1}, x_{n}\right)$ is consistent with $F_{e}$, we are forced to the conclusion that $x=x_{n}$ and $y=x_{n-1}$. Now $\left\{e, x_{n}, x_{n-1}\right\}$ and $\left\{x_{n}, x_{n-1}, x_{n-2}\right\}$ are triangles in $M^{\prime}$, and $\{x, y, z\}=\left\{x_{n}, x_{n-1}, z\right\}$ is a triad, which leads to a contradiction to Corollary 6.3. 
Next we consider the case that $n \leqslant 5$, and $\{x, y, z\} \cap\left\{x_{1}, \ldots, x_{n}\right\}=\left\{x_{2}, x_{4}\right\}$. If $s=2$, then $\left(x_{2}, x_{3}, x_{4}\right)$ must be consistent with $F_{e}$, but $(e, x, y, z)$ contains $x_{2}$ and $x_{4}$ and not $x_{3}$. If $s \neq 2$, then $s=3, n=5$, and $\left(x_{3}, x_{4}, x_{5}\right)$ or its reversal is in $\mathcal{F}_{N}$. However, $(e, x, y, z)$ contains $x_{4}$ and neither $x_{3}$ nor $x_{5}$, so it is impossible for $\left(x_{3}, x_{4}, x_{5}\right)$ to be consistent with $F_{e}$.

Now we know that $\{x, y, z\} \subseteq\left\{x_{1}, \ldots, x_{n}\right\}$. Applying the dual of Proposition 5.6 to $\{x, y, z\}$ and $\left(x_{1}, \ldots, x_{n}\right)$ in $M^{\prime} \backslash e$, we see that $\{x, y, z\}=\left\{x_{i}, x_{i+1}, x_{i+2}\right\}$, where $x_{i}$ is a rim element of $\left(x_{1}, \ldots, x_{n}\right)$, and $i \leqslant n-2$.

6.20.5. $1<i<n-2$.

Proof. Since $x_{1} \notin E(N)$ and $\{x, y, z\} \subseteq E(N)$ it follows that $x_{1} \notin\{x, y, z\}$, so $i>1$. Assume that $i+2=n$. Then $\left\{x_{n-2}, x_{n-1}, x_{n}\right\}$ is a triad in $M^{\prime}$. As $C_{x}^{*}$ is a cocircuit in $M^{\prime}$ by 6.20 .2 , it follows that $C_{x}^{*}-e \neq\left\{x_{n-2}, x_{n-1}, x_{n}\right\}$. As $C_{x}^{*}$ contains at least one of $x$ or $y$, we see that $C_{x}^{*}-e=\left\{x_{n-4}, x_{n-3}, x_{n-2}\right\}$. As $\left(x_{s}, \ldots, x_{t}\right)$ is a fan in $M$, it follows that $s \geqslant n-3$, so some three- or four-element contiguous subsequence of $\left(x_{n-3}, x_{n-2}, x_{n-1}, x_{n}\right)$ is in $\mathcal{F}_{N}$ (up to reversing). If $\{x, y\}=\left\{x_{n-1}, x_{n}\right\}$, then the triangle $\left\{e, x_{n-1}, x_{n}\right\}$ violates orthogonality with $C_{x}^{*}$ in $M^{\prime}$. Assume that $\{x, y\}=\left\{x_{n-2}, x_{n-1}\right\}$. Now $\left\{x_{n-3}, x_{n-2}, x_{n-1}\right\}$ is a triangle in $M \backslash e$, and hence in $M^{\prime}$. As $\{e, x, y\}$ is also a triangle in $M^{\prime}$, it follows that $\left\{e, x_{n-3}, x_{n-2}, x_{n-1}\right\}$ is a $U_{2,4}$-restriction in $M^{\prime}$ that intersects the triad $\{x, y, z\}$. This is a contradiction, so $\{x, y\}=\left\{x_{n-2}, x_{n}\right\}$. Therefore $(e, x, y, z)$ is $\left(e, x_{n-2}, x_{n}, x_{n-1}\right)$ or $\left(e, x_{n}, x_{n-2}, x_{n-1}\right)$. This means any three- or four-element contiguous subsequence of $\left(x_{n-3}, x_{n-2}, x_{n-1}, x_{n}\right)$ contains elements from $(e, x, y, z)$, but cannot be consistent with $F_{e}$, contradicting the fact that $F_{e}$ is in a covering family of $M^{\prime}$.

6.20.6. $\{x, y\}=\left\{x_{i}, x_{i+2}\right\}$, and therefore $z=x_{i+1}$.

Proof. Assume that $\{x, y\}=\left\{x_{i}, x_{i+1}\right\}$. Then $\left\{e, x_{i-1}, x_{i}, x_{i+1}\right\}$ is a $U_{2,4}$-restriction in $M^{\prime}$ that intersects the triad $\{x, y, z\}$, a contradiction. Next assume that $\{x, y\}=\left\{x_{i+1}, x_{i+2}\right\}$. Then $\left\{e, x_{i+1}, x_{i+2}\right\}$ and $\left\{x_{i+1}, x_{i+2}, x_{i+3}\right\}$ are triangles of $M^{\prime}$. We again find that $M^{\prime}$ has a $U_{2,4}$-restriction that intersects a triad.

Note that $F_{e}$ contains $e$ and a fan in $\mathcal{F}_{e}$, so it contains at least four elements.

6.20.7. $\left|F_{e}\right|>4$.

Proof. If $F_{e}$ contains exactly four elements, then $(x, y, z)$ or its reversal is in $\mathcal{F}_{N}$. However, $(x, y, z)$ is $\left(x_{i}, x_{i+2}, x_{i+1}\right)$ or $\left(x_{i+2}, x_{i}, x_{i+1}\right)$, implying that $(x, y, z)$ is not consistent with $\left(x_{1}, \ldots, x_{n}\right)$, although $\{x, y, z\} \subseteq\left\{x_{1}, \ldots, x_{n}\right\}$. This contradicts the fact that either $\left(x_{1}, \ldots, x_{n}\right)$ or $\left(x_{1}, \ldots, x_{n}, e_{1}\right)=\left(e_{m}, \ldots, e_{1}\right)$ is in the covering family $\mathcal{F}_{e}$ (up to reversing).

Let $(e, x, y, z, v)$ be the first five elements of $F_{e}$. If $(y, z)=\left(x_{i}, x_{i+1}\right)$, then $v=x_{i-1}$, for otherwise $\left\{v, x_{i-1}, x_{i}, x_{i+1}\right\}$ is a $U_{2,4}$-restriction in $M^{\prime}$, and $\left\{x_{i}, x_{i+1}, x_{i+2}\right\}$ is a triad. A similar argument shows that if $(y, z)=\left(x_{i+2}, x_{i+1}\right)$, then $v=x_{i+3}$, since otherwise $\left\{v, x_{i+1}, x_{i+2}, x_{i+3}\right\}$ is a $U_{2,4}$-restriction in $M^{\prime}$. 
Assume that $F_{e}$ contains more than five elements. Let $(e, x, y, z, v, w)$ be the first six elements of $F_{e}$. Then $\{z, v, w\}$ is a triad of $M^{\prime} \backslash e$. The previous paragraph shows that $\{z, v, w\}$ cannot be a set of three consecutive elements in $\left(x_{1}, \ldots, x_{n}\right)$, so the dual of Lemma 5.8 shows that $w$ is not in $\left\{x_{1}, \ldots, x_{n}\right\}$. Because $z$ and $v$ are not consecutive in $\left(x_{1}, \ldots, x_{n}\right)$, it follows from Proposition 5.3 that $n \leqslant 5$. Now 6.20 .5 implies that $n=5$, and $i=2$, so $z=x_{3}$. But now the triad $\{z, v, w\}$ and the triangle $\left\{x_{1}, x_{2}, x_{3}\right\}$ violate orthogonality. Hence $F_{e}=(e, x, y, z, v)$.

Either $(e, x, y, z, v)$ is $\left(e, x_{i}, x_{i+2}, x_{i+1}, x_{i+3}\right)$, or it is $\left(e, x_{i+2}, x_{i}, x_{i+1}, x_{i-1}\right)$. Now some three- or four-element subsequence of $\left(x_{i}, x_{i+2}, x_{i+1}, x_{i+3}\right)$ or $\left(x_{i+2}, x_{i}, x_{i+1}, x_{i-1}\right)$ is in $\mathcal{F}_{N}$ (up to reversing). But this subsequence must be equal to $\left(x_{s}, \ldots, x_{t}\right)$ for some values of $s$ and $t$. It is clear that no such subsequence exists, so this completes the proof of Lemma 6.20.

By reversing as necessary, we can assume that $\left(e_{1}, \ldots, e_{m}\right)$ is a fan in $\mathcal{F}_{e}$ and $e_{1} \notin$ $E(N)$. Lemma 6.20 tells us that $e_{1}$ is a rim element, and $\left\{e, e_{1}\right\}$ is contained in a triangle, $T_{e}$, of $M$. By Lemma 6.18, $M \backslash e / e_{1}$ is 3 -connected and has $N$ as a minor. As $|E(M)|-$ $|E(N)|>2$, we let $x$ be an element in $E\left(M \backslash e / e_{1}\right)-E(N)$. First we assume that the fan in $\mathcal{F}_{e}$ that contains $x$ is disjoint from $\left(e_{1}, \ldots, e_{m}\right)$. Let $\left(x_{1}, \ldots, x_{n}\right)$ be this fan, where we can reverse as necessary and assume that $x=x_{1}$. Then $x_{1}$ is a rim element of $\left(x_{1}, \ldots, x_{n}\right)$ and $\left\{e, x_{1}\right\}$ is contained in a triangle, $T_{x}$. Note that $N$ is a minor of $M \backslash e / e_{1} / x_{1}$, for $\left\{x_{2}, x_{3}, x_{4}\right\}$ is a codependent triangle in $M \backslash e / e_{1} \backslash x_{1}$. This means $T_{e} \neq T_{x}$, for otherwise $e$ is a loop in $M / e_{1} / x_{1}$, and this matroid has $N$ as a minor, so is connected. Let $C$ be a circuit of $M \backslash e$ contained in $\left(T_{e} \cup T_{x}\right)-e$ that contains $e_{1}$. If $|C|=3$, then $M \backslash e / e_{1}$ contains a parallel pair, contradicting its 3-connectivity, so $C=\left(T_{e} \cup T_{x}\right)-e$, and therefore $x_{1} \in C$. By orthogonality with $\left\{e_{1}, e_{2}, e_{3}\right\}$ we deduce that $C$ contains an internal element from $\left(e_{1}, \ldots, e_{m}\right)$ (note $m \geqslant 4$ as $\left(e_{1}, \ldots, e_{m}\right)$ contains $e_{1}$ and a fan from $\left.\mathcal{F}_{N}\right)$, and this element is in $E(N)$ by Lemma 6.17. By orthogonality with $\left\{x_{1}, x_{2}, x_{3}\right\}$, we see that $C$ also contains an element from $\left(x_{2}, \ldots, x_{n}\right)$ that is in $E(N)$. Thus $C-\left\{e_{1}, x_{1}\right\}$ is a parallel pair in $M \backslash e / e_{1} / x_{1}$, and in $N$, a contradiction.

Now the only elements in $E\left(M \backslash e / e_{1}\right)-E(N)$ belong to the same fan of $\mathcal{F}_{e}$ as $e_{1}$. By Lemmas 6.17 and 6.20, we can assume $\left(e_{1}, \ldots, e_{m}\right)$ is a fan in $\mathcal{F}_{e}$ such that $e_{1}$ and $e_{m}$ are rim elements not in $E(N)$, and that $N=M \backslash e / e_{1} / e_{m}$. Let $T_{1}$ and $T_{m}$ be triangles of $M$ such that $\left\{e, e_{1}\right\} \subseteq T_{1}$ and $\left\{e, e_{m}\right\} \subseteq T_{m}$. Note that $T_{1} \neq T_{m}$, as $M / e_{1} / e_{m}$ has $N$ as a minor, and is therefore connected. Let $C$ be a circuit of $M \backslash e$ contained in $\left(T_{1} \cup T_{m}\right)-e$ that contains $e_{1}$. Then $|C|=4$, or else $M \backslash e / e_{1}$ contains a parallel pair. Hence $e_{m} \in C$. Therefore $C-\left\{e_{1}, e_{m}\right\}$ is a parallel pair in $M \backslash e / e_{1} / e_{m}=N$, a contradiction. Now we have completed the proof of Theorem 6.10.

\section{Acknowledgements}

We thank Geoff Whittle for valuable discussions and the referee for a careful reading. 


\section{References}

[1] C. Chun, D. Chun, B. Clark, D. Mayhew, G. Whittle, and S. H. M. van Zwam. Computer-verification of the structure of some classes of fragile matroids. arXiv: 1312.5175

[2] J. Geelen, B. Gerards, and G. Whittle. Towards a matroid-minor structure theory. In Combinatorics, complexity, and chance, volume 34 of Oxford Lecture Ser. Math. Appl., pp. 72-82. Oxford Univ. Press, Oxford (2007).

[3] J. Geelen, J. Oxley, D. Vertigan, and G. Whittle. Weak maps and stabilizers of classes of matroids. Adv. in Appl. Math. 21 (1998), no. 2, 305-341.

[4] J. F. Geelen, A. M. H. Gerards, and A. Kapoor. The excluded minors for GF(4)representable matroids. J. Combin. Theory Ser. B 79 (2000), no. 2, 247-299.

[5] R. Hall, D. Mayhew, and S. H. M. van Zwam. The excluded minors for near-regular matroids. European J. Combin. 32 (2011), no. 6, 802-830.

[6] S. R. Kingan and M. Lemos. Almost-graphic matroids. Adv. in Appl. Math. 28 (2002), no. 3-4, 438-477. Special issue in memory of Rodica Simion.

[7] S. R. Kingan and M. Lemos. Strong splitter theorem. arXiv:1201.4427

[8] D. Mayhew, G. Whittle, and S. H. M. van Zwam. Stability, fragility, and Rota's Conjecture. J. Combin. Theory Ser. B 102 (2012), no. 3, 760-783.

[9] J. Oxley. Matroid theory. Oxford University Press, New York, second edition (2011).

[10] J. Oxley and H. Wu. On the structure of 3-connected matroids and graphs. European J. Combin. 21 (2000), no. 5, 667-688.

[11] J. G. Oxley. A characterization of a class of nonbinary matroids. J. Combin. Theory Ser. B 49 (1990), no. 2, 181-189.

[12] R. A. Pendavingh and S. H. M. van Zwam. Confinement of matroid representations to subsets of partial fields. J. Combin. Theory Ser. B 100 (2010), no. 6, 510-545.

[13] K. Truemper. A decomposition theory for matroids. VI. Almost regular matroids. J. Combin. Theory Ser. B 55 (1992), no. 2, 253-301. 